\title{
A step-by-step guide to strengthening sexual violence services in public health facilities: Lessons and tools from sexual violence services in Africa
}

Jill Keesbury

Population Council

Jill Thompson

Follow this and additional works at: https://knowledgecommons.popcouncil.org/departments_sbsr-rh

Part of the Demography, Population, and Ecology Commons, Domestic and Intimate Partner Violence Commons, Family, Life Course, and Society Commons, Gender and Sexuality Commons, International Public Health Commons, Medicine and Health Commons, and the Women's Health Commons How does access to this work benefit you? Let us know!

\section{Recommended Citation}

Keesbury, Jill and Jill Thompson. 2010. "A step-by-step guide to strengthening sexual violence services in public health facilities: Lessons and tools from sexual violence services in Africa." Lusaka: Population Council. 
A STEP-BY-STEP GUIDETO

STRENGTHENING SEXUALVIOLENCE

SERVICES IN PUBLIC HEALTH

FACILITIES:

LESSONS AND TOOLS FROM SEXUAL

VIOLENCE SERVICES IN AFRICA

26 November 2010

This publication was produced for review by the United States Agency for International

Development. It was prepared by jill Keesbury (Population Council/Zambia) and Jill Thompson (consultant). 



\section{A STEP-BY-STEP GUIDE TO STRENGTHENING SEXUAL VIOLENCE SERVICES IN PUBLIC HEALTH FACILITIES:}

\section{LESSONS AND TOOLS FROM SEXUAL VIOLENCE SERVICES IN AFRICA}

\section{JILL KEESBURY, POPULATION COUNCIL/ZAMBIA JILL THOMPSON, INDEPENDENT CONSULTANT}




\section{ACKNOWLEDGEMENTS}

The authors wish to acknowledge the contributions of numerous partners across Africa. This guide is the product of their commitment to strengthening services for sexual violence survivors and to developing innovative approaches to providing services in low-resource settings. Many of the experiences cited in this guide are drawn from partners that are members of the Population Council's African Regional genderbased violence network, which is supported by the Swedish-Norwegian HIV/AIDS Team in Africa.

This report was made possible through support provided by the United States Agency for International Development (USAID) under the terms of Contract No. GHS-I-00-07-00011-00. The contents are the responsibility of the authors and do not necessarily reflect the views of USAID or the United States Government.

\footnotetext{
18 Population Council The Population Council conducts research worldwide to improve policies, programs, and products in three areas: HIV and AIDS; poverty, gender, and youth; and reproductive health. www.popcouncil.org
}

Published in November 2010.

Copyright @2010. United States Agency for International Development.

Suggested citation: Keesbury, Jill and Jill Thompson. 2010. "A step-by-step guide to strengthening sexual violence services in public health facilities: Lessons and tools from sexual violence services in Africa." Lusaka: Population Council.

The authors' views expressed in this publication do not necessarily reflect the views of the United States Agency for International Development or the United States Government. 


\section{TABLE OF CONTENTS}

ACRONYMS

INTRODUCTION I

Purpose of this step-by-step guide I

Target audience: Who should use this guide? $\quad 2$

Ethical considerations in providing SV services $\quad 2$

What do we mean by "comprehensive SV care"? $\quad 4$

Comprehensive services for children and adolescents $\quad 5$

$\begin{array}{ll}\text { Where can comprehensive SV services be provided? } & 6\end{array}$

\section{STEP I: COLLECTING THE INFORMATION NEEDED TO INFORM SERVICE} STRENGTHENING AND INTEGRATION 9

A. Laying the groundwork for successful program implementation: stakeholder consultations 9

B. Conduct a facility inventory to assess the facility's physical capacity to provide services II

$\begin{array}{ll}\text { C. Hold focus group discussions with health care providers } & 12\end{array}$

D. Identify legal requirements for proper forensic evidence collection and documentation $\quad 13$

E. Conduct community-based assessments $\quad$ I5

STEP 2: ESTABLISHING THE REGULATORY AND INFRASTRUCTURAL FRAMEWORK FOR SERVICES $\quad 16$

A. Ensure that facility-level guidelines for SV service delivery are in place $\quad 16$

B. Minimize multiple referral points $\quad 18$

C. Ensure that SV examination points are stocked with necessary supplies, equipment and guidelines 19

D. Ensure adequate record-keeping procedures are in place for SV cases $\quad 20$

E. Reduce or eliminate fees for medical and medico-legal services 21

STEP 3:TRAINING HEALTH CARE PROVIDERS

A. Core elements to be included in SV training programs $\quad 22$

B. Identify the service providers to be trained, departments to be included in SV service provision 24

C. Conduct cross-training with referral providers, including the police and legal sectors 26 


\section{STEP 5: DEVELOPING REFERRAL LINKAGES TO OTHER SERVICE} PROVIDERS

A. Conduct participatory community mapping of all referral points

B. Develop a formal referral directory and protocol for out-referrals and post in the SV examination room

C. Develop an algorithm of care (flow diagram) that includes external referrals

D. Consider developing referral systems to facilitate and track use of referral services

\section{STEP 6: STRENGTHENING LINKAGES BETWEENTHE HEALTH} FACILITY AND COMMUNITIES TO INCREASE TIMELY UTILIZATION OF SERVICES

A. Identify community leaders and partners 36

B. Develop communication materials for community and clinic-based awareness creation 36

C. Integrate SV messages into existing health service outreach activities 37

D. Create clinic-community linkages to sensitize communities and increase service utilization 37

STEP 7: MONITORING AND EVALUATING THE PROGRAM 39
A. Identify appropriate indicators

B. Identify appropriate data collection/capture tools 42

C. Create community monitoring systems 43

D. Ongoing quality assurance and supervision 44

STEP 8: ENSURING ADEQUATE RESOURCES

A. Mobilize resources for one-time program start-up costs $\quad 45$

B. Ensure availability of resources for ongoing recurrent costs 46

CONCLUSION: PROMOTING COUNTRY OWNERSHIP, SUSTAINABILITY AND SCALE-UP $\quad 48$

A. Ensuring country ownership and sustainability 48

B. Considerations for taking pilot SV programs to scale 49 
HELPFUL RESOURCES

REFERENCES

APPENDIX I SAMPLE CONSENT FORM FOR MEDICO-LEGAL

EXAMINATION

APPENDIX 2 SAMPLE FACILITY INVENTORYTOOL

APPENDIX 3 SAMPLE GUIDE FOR FGDS WITH SERVICE PROVIDERS

APPENDIX 4 SAMPLE INFORMED CONSENT FORM FOR PARTICIPATION IN FGDS

APPENDIX 5 MEDICO-LEGAL FORM DEVELOPED FOR USE INTHE SGBV INITIATIVE

APPENDIX 6 SAMPLE COMMUNITY FGDTOOL

APPENDIX 7 MINIMUM SUPPLIES NEEDED TO PROVIDE SV SERVICES

APPENDIX 9 SAMPLE CLIENT FLOW CHART, MULAGO HOSPITAL, KAMPALA, UGANDA

APPENDIX IO AWARENESS-RAISING POSTER DEVELOPED FOR USE WITHIN UGANDAN COMMUNITIES

APPENDIX I I AWARENESS-RAISING POSTERS DEVELOPED FOR USE WITHIN UGANDAN HEALTH FACILITIES 



\section{ACRONYMS}

AIDS

ART

ASAZA

AZT

CBOs

CDC

CEO

CRS

CSA

DCI

DNA

EC

$\mathrm{FBOs}$

FGD

FI

GBV

GRIP

HCT

HIV

HPI

ICAP

IGWG

IPPF

IPV

IUD

LVCT

$\mathrm{M} \& \mathrm{E}$

MJAP

$\mathrm{MOH}$

MRC

NGO

NUMAT

OB-GYN

OPD

PEP
Acquired Immune Deficiency Syndrome

Antiretroviral Therapy

A Safer Zambia

Azidothymidine

Community-based Organizations

Centers for Disease Control

Chief Executive Officer

Catholic Relief Services

Child Sexual Abuse

Drew Cares International

Deoxyribonucleic Acid

Emergency Contraception

Faith-based Organization

Focus Group Discussion

Facility Inventory

Gender-based Violence

Greater Nelspruit Rape Intervention Programme

HIV Counseling and Testing

Human Immunodeficiency Virus

Health Policy Initiative

International Center for AIDS Care and Treatment Programs

International Gender Working Group

International Planned Parenthood Federation

Intimate Partner Violence

Intra-uterine Device

Liverpool VCT, Care and Treatment

Monitoring and Evaluation

Mulago-Mbarara Teaching Hospitals' Joint AIDS Program

Ministry of Health

Medical Research Council

Non-governmental Organization

Northern Uganda Malaria, Tuberculosis and HIV AIDS Program

Obstetrics and Gynecology

Outpatient Department

Post-exposure Prophylaxis 
PEPFAR

RHRC

SGBV

SGBV Initiative

STIs

SV

SVAC

SVRI

TA

TTCs

TVEP

UNHCR

UPDF

USAID

VCT

WHO
President's Emergency Plan for AIDS Relief

Reproductive Health Response in Conflict

Sexual and Gender-based Violence

Special Initiative on Sexual and Gender-based Violence

Sexually Transmitted Infections

Sexual Violence

Sexual Violence Advisory Committee

Sexual Violence Research Initiative

Technical Assistance

Thuthuzela Care Centres

Thohoyandou Victim Empowerment Programme

United Nations High Commissioner for Refugees

Uganda People's Defense Force

United States Agency for International Development

Voluntary Counseling and Testing

World Health Organization 


\section{INTRODUCTION}

Sexual violence (SV) is a serious health and human rights problem across Africa that disproportionately affects women and girls. Survivors require comprehensive and sensitive care to mitigate the negative health consequences of SV, minimize psychological trauma and promote long-term reintegration and recovery.

Despite high levels of sexual violence and a growing recognition of the need to improve the management of rape and sexual assault, many public health facilities in Africa do not currently have capacity to provide comprehensive, patient-centered SV care to adult and child survivors. Public facilities face numerous challenges including lack of specialized SV training, general understaffing and lack of resources, and gaps in essential clinical services, such as emergency contraception (EC), psychosocial counseling and post-exposure prophylaxis (PEP) for HIV. ${ }^{1-4}$ Community and family attitudes, feelings of shame or self-blame, low awareness about SV and SV services, and lack of funds for transportation or medical services create further challenges that prevent or inhibit many survivors from seeking essential care. ${ }^{5}$ Despite these challenges, there is a growing base of evidence that targeted, systemic interventions at health facilities-particularly when combined with multi-sectoral approaches and community outreach and involvement—can have a significant, sustainable impact on the availability and quality of SV care, even in low-resource settings., ${ }^{3,6,7}$

\section{PURPOSE OF THIS STEP-BY-STEP GUIDE}

The purpose of this document is to provide practical guidance on the steps necessary to establish and strengthen SV services within existing public health facilities, improve linkages to other sectors, and engage local communities around issues of sexual violence. It includes the following tips, resources and tools that will help partners and stakeholders:

- Assess and document the existing state of SV services at the cooperating health facility as a basis for designing the intervention and establishing a baseline for monitoring and evaluation;

- Plan and implement sustainable improvements in service delivery;

- Promote multi-sectoral coordination and programming;

- Develop and/or strengthen linkages with other SV service providers;

\section{CASE STUDY:}

\section{THE PEPFAR SGBV INITIATIVE}

In response to growing concerns over sexual violence in Africa and its impact on HIVIAIDS, the U.S. President's Emergency Plan for AIDS Relief (PEPFAR) initiated a Special Initiative on Sexual and Gender-based Violence (SGBV Initiative) in 2007. The purpose of the SGBV Initiative was to contribute to the evidence base on SV programming in Africa, while strengthening health services for survivors of sexual violence through the implementation of a comprehensive model of care in participating PEPFAR partner facilities. The SGBV Initiative was implemented at 18 pilot sites in Rwanda and Uganda, which included local health centers, regional and district hospitals, and military hospitals.

At each site, PEPFAR partners worked with local health providers and community stakeholders to pilot or enhance site-specific service delivery models based on the needs, preferences, and capacities within their health care setting and based on government policies and community practices. Lessons learned from this initiative are provided throughout this guide.

For more information see: Keesbury, J. and Elson, L. 2010. PEPFAR special initiative on sexual and gender-based violence: Final evaluation." Lusaka: Population Council. 
- Engage local communities to identify and address barriers to reporting, facilitate referrals, and provide longer-term support to SV survivors; and

- Monitor and evaluate implementation and program impact.

Included in the guide are a number of practical resources and tools developed and validated by the SGBV Initiative, which can be adapted as needed for use in other settings.

\section{TARGET AUDIENCE:WHO SHOULD USE THIS GUIDE?}

This guide is for all SV service providers, governments and non-governmental organizations (NGOs) interested in strengthening SV services in their countries and communities. It is aimed particularly at PEPFAR partners currently working with public health facilities in the context of HIV and AIDS, voluntary counseling and testing (VCT) and antiretroviral therapy (ART) programs, and service providers and partners involved in existing reproductive, child and/or maternal health programs. It can also be used by government health ministries, international and non-governmental organizations, and PEPFAR country teams to develop and plan country-level SGBV initiatives. The guidance covers both clinical services and community outreach components, as well as steps that can be taken in conjunction with other key stakeholders to implement comprehensive SV services and improve multi-sectoral coordination.

The steps outlined below can be used to establish and/or strengthen comprehensive SV services at all levels of the health system. Specialized "one-stop centers" for SV care are not a prerequisite for providing comprehensive care, and this guide outlines the necessary steps for delivering such services in a wide range of health care settings.

This guide was developed based on research, materials and lessons learned from SV programs in Rwanda, Uganda, Ethiopia, Malawi, Kenya, South Africa, and Zambia. The guidance is therefore particularly applicable to low-resource settings in Africa, most especially within public sector health facilities. Much of this guidance, however, can be applied to low- and mid-resource settings in other regions, as it addresses the basic issues associated with establishing and maintaining comprehensive care for SV survivors.

\section{ETHICAL CONSIDERATIONS IN PROVIDING SV SERVICES}

SV survivors are most often in a compromised and highly vulnerable position when they seek services. From inception, programs must recognize the vulnerability of these clients and ensure that treatment does not cause further trauma. The following principles should guide the planning and implementation of all SV programs. ${ }^{8,9}$

- Health care providers should be appropriately trained and skilled in managing sexual assault patients. All providers responsible for SV patients should undergo basic sensitization training and orientation to the SV clinical and referral protocols, as well as specialized training on medico-legal examination and PEP. To the extent possible, the health center should identify and train dedicated practitioners (doctors, forensic nurses, or clinical officers) to provide SV care and management, particularly in cases involving young children. 
- Patients must be treated with respect and sensitivity to protect against secondary victimization. This may include, for example, giving SV cases priority (front of the queue), displaying empathy and avoiding judgmental or blaming attitudes or questions, protecting the patient's privacy, and providing clear information and explaining processes in a language the survivor can understand. Survivors should always be given the option to have a trusted person present during the examination. If a female health provider is not available to conduct the examination of a female survivor, a female health worker should be present in the room.

- Patient confidentiality must be respected and maintained at all times. This requires appropriate sensitization of staff, private counseling and waiting areas, as well as systems and policies to govern access to patient files. Consent should always be obtained from the survivor before sharing information with other service providers, including police and referral organizations.

- Informed consent should be obtained from the patient (and the patient's caregiver, if under age) prior to treatment and at each stage of the examination. Written consent should be obtained for VCT and for release of information to the police or other third parties (such as counselors or data management personnel). See Appendix 1 for a sample consent form.

- The health and welfare of the patient should take precedence over collection of evidence. Crisis containment, treatment of serious injuries and assessment, prevention and management of HIV, STIs and pregnancy is of primary importance. The welfare of patients includes ensuring that patients are able to maintain their dignity after the assault and do not feel coerced, humiliated or further traumatized by the process of seeking medico-legal services. Survivors, including children of any age, should never be forced to undergo rape examination against their will, unless the examination is necessary for medical care. $^{8}$

- Reporting to police should not be a prerequisite for obtaining medical care. The survivor's decision regarding involvement of police should be respected at all times. A SV survivor should never be forced or pressured to report the sexual assault or to undergo the medico-legal examination as a condition of receiving other essential care. It is common for health care workers and police to tell survivors that they must report to police and obtain the police form before the health facility will conduct the examination. Reporting is also often tied to payment of fees; the hospital may only agree to provide free services if the patient has reported a sexual assault to police and is in possession of the official form. In most cases, these restrictions appear to be matters of procedure rather than legal requirements, and therefore can be changed at facility level. They may even be inconsistent with national policy. These issues should be discussed and interrogated as part of the training and development of protocols, particularly if they appear to be inhibiting access to services at the intervention site. Police forms may be kept at the health facility for survivors who present first to the hospital, so that survivors need not be redirected to police before receiving EC, PEP or other health services. A survivor should be offered all available services, even if a doctor is not available to sign medico-legal forms.

- In the case of children, the best interests of the child should always be the paramount consideration. Clinical, psychosocial, and referral protocols should include specific procedures for children. ${ }^{10}$ Efforts should be made to ensure that SV facilities and services are "child-friendly" and that health providers are trained to address the special needs of children, adolescents, and their families. Children should never be forced to undergo medical examination unless necessary for medical treatment. A trained social worker should be contacted and present before the child gives his or her 
statement to police. Health providers must also be aware of their legal obligations to report cases of child sexual abuse and procedures for removing a child to a place of safety if required.

\section{- Research involving survivors should be conducted only when data cannot be gathered from} other sources, and must not place the survivor at risk of further emotional or physical trauma. Evaluations of health services should limit data collected directly from survivors, particularly at the time of initial reporting. If data from the survivor is required to assess program effectiveness (i.e., PEP adherence, client satisfaction with services), recruitment should occur several weeks after all services (including psychosocial support) have been provided. ${ }^{6}$ Interviews that focus on the assault should be avoided, and if necessary, conducted only in the presence of a trained counselor. Researchers must also be mindful that household or community-based interviews can inadvertently put the survivor at risk, especially if a member of the household perpetrated the violence. ${ }^{11}$ To the extent possible, data for monitoring and evaluation purposes should be obtained from other sources, such as interviews with service providers, facility inventories, observations, and review of charts, patient registers and other documentation.

\section{WHAT DOWE MEAN BY “COMPREHENSIVE SV CARE”?}

The term "sexual violence" refers to all forms of sexual assault and abuse of women, men, adolescents,

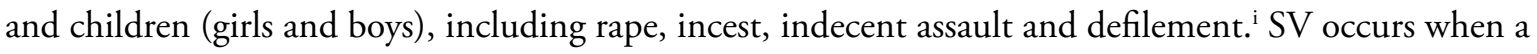
person uses psychological pressure, abuse of authority, threats or physical force against another person for sexual purposes, whether or not the act constitutes a criminal offence under domestic law.

Comprehensive SV care meets the range of medical, legal, and psychosocial needs of the SV survivor, from the first point of contact through the final stages of recovery and reintegration. This includes: comprehensive medical management by health care providers (including prevention of HIV), short and long-term psychosocial support, and legal assistance to help the survivor access justice. Table 1 outlines each sector's contribution to the core elements of comprehensive care. Many of these services need to be provided as soon as possible following SV and no later than 72 hours following the assault, including PEP, forensic evidence collection and EC (within 120 hours).

Comprehensive care necessarily requires a multi-faceted, multi-sectoral response, involving a range of stakeholders, service providers and the community at large. Although health facilities can strengthen their services without reference to other stakeholders, clinical management of SV is most effective when it is situated within this broader, multi-sectoral response, and the various components and stakeholders are effectively coordinated.

In some cases, comprehensive care can be provided in one location that houses representatives from all the sectors, which is sometimes referred to as a "one-stop center." When this is not possible, comprehensive care must be facilitated through an active and effective referral network.

'The term "defilement" is used in many African countries to refer to sexual intercourse with a girl under a certain age, and is often used interchangeably with child sexual abuse. 
Table I Core components of a comprehensive response to SV

\begin{tabular}{|c|c|}
\hline Sector & Key components of response \\
\hline \multirow{5}{*}{ Health } & HIV diagnostic testing and counseling and PEP \\
\hline & Prophylaxis for sexually transmitted infections \\
\hline & Vaccination for Hepatitis B and Tetanus \\
\hline & Trauma counseling \\
\hline & Referrals to/from police and social support sectors \\
\hline \multirow{4}{*}{ Police/Justice } & Statement-taking and documentation \\
\hline & Criminal investigation \\
\hline & Witness preparation and court support \\
\hline & Referrals to/from health and social support sectors \\
\hline \multirow{5}{*}{ Social Support } & Assessment of the need for psychosocial services \\
\hline & Referral to short-term or long-term psychosocial support services \\
\hline & Provision of safe housing, relocation services, if required \\
\hline & Reintegration into family/household, if required \\
\hline & Long-term psychosocial counseling and rehabilitation \\
\hline
\end{tabular}

\section{COMPREHENSIVE SERVICES FOR CHILDREN AND ADOLESCENTS}

Children and adolescents constitute a significant proportion of survivors who present for health and related SV services in Africa. In Rwanda and Uganda, the mean age of survivors seeking services under the SGBV Initiative was 14 and 15 years old, respectively. The vast majority of these survivors were girls. 3,5

Despite the fact that children and adolescents constitute the majority of survivors seeking care in many countries, many facilities do not include child-specific services, equipment, procedures or training in their SV programs. ${ }^{3,5}$ When establishing an SV intervention, special care must be taken to ensure that the unique needs of child survivors (as well as other survivors with special needs) are fully taken into account. For example:

- Making an effort to create a child-friendly space where children and adolescents can relax and feel safe;

- Ensuring that health providers are specially trained to conduct medico-legal examinations of children and accurately interpret the findings; 
- Ensuring that health providers are familiar with different treatment regimens for children and that pediatric medicines are available at all times;

- Understanding the unique dynamics of child sexual abuse and the challenges of disclosure;

- Recognizing that having a parent present in the interview or examination is not always appropriate/ understanding of how to manage family dynamics, attitudes and expectations that may not be in the best interest of the child or adolescent survivor;

- Ensuring that clinical protocols and forms specifically address areas where the care and treatment of children differs from those of adults;

- Understanding health care workers' legal obligations with respect to child survivors, and legal provisions regarding informed consent;

- Identifying relevant services for children, adolescents and caregivers available in the community;

- Addressing children, adolescents and caregivers in targeted outreach and awareness efforts.

Although this guide does not specifically focus on children and adolescents, many of the guidelines, training materials and other resources referenced contain specific sections on children and child sexual abuse that may be utilized in connection with program planning and training of service providers.

\section{WHERE CAN COMPREHENSIVE SV SERVICES BE PROVIDED?}

Comprehensive SV services can be provided at all levels of the public health care system, from local health centers and clinics to national referral hospitals. Some aspects of care can even be provided in nontraditional sites such as police stations, as demonstrated recently in Zambia's Copperbelt Province. There, specialized police officers from the Victim Support Units were trained to provide EC to SV survivors and refer them to health facilities for further care. ${ }^{12}$

The level of SV services that can be provided at a given facility will vary depending on the capacity and resources of the facility. National health policy and legal considerations may also play a role. When planning your intervention, you will need to determine what aspects of comprehensive care can be provided at the facility, and when you will need to refer survivors to resources outside the facility. To the extent possible, all emergency services (including crisis counseling) should be provided on-site at the facility.

\section{Health Centers}

Health centers and clinics are primary care facilities that are located close to the community. Generally, they do not have capacity to treat serious injuries or complications requiring surgical theatres or specialized care, and may not have laboratory or ART facilities on site. However, they can provide many core SV services, including HIV prevention, when properly trained and equipped. For example, health centers may be able to offer urgent care services, such as crisis counseling, EC, and PEP to maximize the efficacy of these treatments pending transport to the local or regional hospital. This is particularly important to consider in rural areas where the community may be located far from the hospital, and in cases which are running up against the 72-hour deadline for starting PEP. Health centers can also play a role in providing more accessible follow-up services after the patient's initial visit to the hospital. These 
may include, for example, monitoring and support for PEP adherence, monitoring and treatment of STIs, repeat doses of Hepatitis B vaccine, follow-up HIV testing, and emotional/psychosocial support. An advantage of health centers is that they are generally located closer to the communities where survivors live, but often require an active referral network to ensure that all elements of comprehensive care are provided.

In the SGBV Initiative, seven of the eighteen intervention sites were health centers, staffed primarily by nurses. In all of these sites, health providers were able to offer services such as medical examination, treatment for injuries and STIs, HIV testing, PEP and EC. ${ }^{5}$

Legal considerations may be a factor in determining whether health centers can provide medico-legal services in SV cases. For example, while most essential SV services (aside from surgery) can be provided effectively by appropriately trained nurses and/or clinical officers, national laws may only allow qualified medical officers (doctors) to conduct and/or certify forensic examinations and testify in court ${ }^{\mathrm{ii}}$. In countries where this is the case, health centers without doctors may need to refer SV patients to larger facilities for medico-legal examination. This can result in delays in receiving emergency services because survivors who report to lower-level health facilities are referred to hospitals with doctors on duty. ${ }^{3} \mathrm{~A}$ particular challenge at health centers in Uganda was the lack of doctors to sign the medical examination forms. Although nurses were able to conduct the examinations and provide treatment, only medical doctors were legally allowed to certify the forms. ${ }^{5}$ This created delays in completing the forms and returning them to survivors and police. In some cases doctors did not cooperate in signing the forms, as they did not want the responsibility of going to court if the case proceeded to trial.

\section{Hospitals}

All of the SV services that can be provided in health centers can also be provided in district and regional hospitals. In addition, hospitals are generally better equipped to provide 24-hour emergency services, treatment for serious injuries, and laboratory services. In many countries, hospitals are also equipped to provide specialized services for HIV/AIDS, including ART. They are also more likely to have doctors available who can complete the required medico-legal documentation. For these reasons, local clinics and police tend to refer survivors to hospitals for medical care, even though the majority of survivors report first to police or local health centers.

Within hospitals, SV services can be centralized in one location or distributed throughout the hospital. Most often these services are provided in the outpatient, emergency or gynecology departments, or in a dedicated reproductive health, HIV/STI or ART clinic. In some facilities, such as Uganda's Mulago hospital, SV services are centralized in the gynecology department, where all necessary care and services are provided 24 hours per day. ${ }^{5}$ In many others, however, survivors are required to seek services from

\footnotetext{
${ }_{i i}^{i}$ Forensic nurses provide the full range of services for SV patients at several Thuthuzela Care Centers (TCCs) in South Africa, including medical examination and PEP. This is only the case, however, in those provinces which recognize forensic nurses as medical experts qualified to testify in court (i.e., Gauteng, Western Cape). The Child Sexual Abuse (CSA) centers in Lusaka and Livingstone, Zambia, rely primarily on nurses and clinical officers to provide SV services, under the supervision of a dedicated medical officer. While the clinical officer may conduct the examination, Zambian law requires that a doctor sign the official police medical examination form. Therefore, local health clinics without doctors currently must refer patients to the hospital for an official SV examination.
} 
numerous points of contact across the hospital, some of which operate only during business hours. One study in South Africa documented that 80 percent of survivors saw six or more providers during a single visit, and that HIV-related services were not available after hours. An intervention to rationalize services within the hospital reduced that proportion to 54 percent, ensured 24-hour access to VCT and PEP and demonstrated significant increases in care provided and survivor satisfaction. ${ }^{6}$ Thus, it is advisable to limit referrals within the hospital as much as possible to ensure access and quality of care.

\section{"One-stop Centers"}

The idea of the "one-stop center" model is to bring multi-sectoral stakeholders together to provide all essential SV services in one place. In addition to offering clinical services, the one-stop typically has a police officer on-site or on call to open dockets and/or take statements at the center, a social worker, nurse, and/or lay counselors to provide crisis counseling and psychosocial support, and ideally, someone to liaise with the prosecutor and monitor the cases going to trial. The one-stop may also provide or refer clients for additional services such as psychological counseling, emergency shelter, or ART. One-stop SV centers tend to be co-located inside or on the grounds of existing hospitals or health centers, although there are some examples of "stand-alone" clinics or community-based sites.

The advantage of the one-stop model is that it can potentially provide more efficient and coordinated services, and a full range of services necessary for comprehensive care. However, they also tend to require significantly more space and resources to operate, including dedicated staff and a site manager or services coordinator. In addition, questions about cost-effectiveness and sustainability remain unanswered, particularly in rural areas where the client load is generally small. 


\title{
STEP I: COLLECTING THE INFORMATION NEEDED TO INFORM SERVICE STRENGTHENING AND INTEGRATION
}

\begin{abstract}
When should this be done? At the outset of program planning.
What staff are needed? At minimum, I-2 program managers can conduct the facility assessments and identify legal requirements. Program staff must carry out the stakeholder consultations. External facilitators are recommended for provider focus group discussions, and providers can conduct community-based assessments.

Other resources required: Limited costs include refreshments for participants in consultations and focus groups, transportation to sites.
\end{abstract}

Once you have established a need for SV services, the first step in the planning process is to learn as much as possible about how SV services are currently provided in the health facility where you plan to work, the attitudes, awareness and concerns of the community, other SV services and resources in the community and the linkages that exist between the health facility and other service providers.

This can be accomplished through preliminary consultations with key stakeholders, structured or semistructured interviews with health providers, staff, and other service providers/stakeholders; observations; focus group discussions (FGDs) with community members and health providers; and the use of a facility inventory (FI) (see FGD guides and FI assessment tools below). If possible, it is best to use standardized assessment tools to allow comparison pre- and post-intervention (and for ongoing monitoring and evaluation for management purposes). If more than one facility is involved in the program, standardized tools will allow more accurate comparison between sites.

\section{A. LAYING THE GROUNDWORK FOR SUCCESSFUL PROGRAM IMPLEMENTATION: STAKEHOLDER CONSULTATIONS}

Active and early engagement with key stakeholders, including facility management, government ministries, community leaders, NGOs and advocacy groups, is essential to lay the groundwork for a successful SV intervention. This consultation process should begin at the planning stages and continue throughout the assessment, implementation and monitoring stages of the project. 
Early engagement is critical for several reasons: First, it is important to build a sense of ownership and commitment to the program at the facility and district level. Regardless of who is providing funding and/ or technical assistance, the program must be "owned," "championed," and eventually managed by the health facility itself, ideally with support from the Ministry or Department of Health. If the program is perceived as an "outside" project, it is less likely to be sustained in the long term. Second, it is important to bring the management of the facility and the key medical supervisors (i.e., head doctor/medical superintendent, head nurse/nursing superintendent, head pharmacist, laboratory manager, human resources department) on board to discuss what role they and their staff will be expected to play in the implementation of the program. This includes what resources (financial, facilities, staff, and institutional support) will be provided from which sources (internal and external) as well as staffing requirements and the procedures and protocols to be followed for consultation and decision-making.

As part of this process, partners should consider whether it will be important in a particular setting to formalize the arrangement in an MOU or service level agreement. Service-level agreements can be helpful for long-term management and accountability, but can also slow down the initial start-up process as they may require negotiation and buy-in at higher levels.

In consultation with facility management, partners should also identify other key stakeholders who should be consulted prior to implementation, e.g., government departments of ministries at various levels, or particular units or departments in the facility such as outpatient, gynecology and pediatric departments, pharmacy, or laboratory. Partners should consider, in particular, which levels of government (national or local) control questions regarding policy, budget, staffing and supplies. This may depend on whether the health facility provides primary, secondary or tertiary care. It is also important to identify and consult stakeholders outside the health sector (police, social services, local leaders) to strengthen inter-sectoral coordination and build a sense of joint ownership over all aspects of the program.

TIP: Many projects find it helpful to establish an SV services advisory/management committee to provide oversight, technical input, and assist with problem solving. The committee should include representatives from senior management, medical supervisors, and key SV health providers, if possible. To promote multi-sectoral coordination and service delivery, it is also recommended to include senior representatives from police and/or social services, as well as key community or NGO partner(s). In South Africa's Refentse program, a key intervention was the establishment of a Sexual Violence Advisory Committee (SVAC), comprised of the hospital CEO, health care providers, medical management, social workers, and representatives from the local police station. The SVAC helped encourage buy-in and support from the relevant stakeholders, and also contributed to the design, implementation and monitoring of the intervention. ${ }^{6}$ In the Zambia EC Program, a multi-sectoral steering committee formed to guide the intervention, also created collaboration among the key stakeholders by creating a high-level forum for the identification and resolution of challenges in providing comprehensive care. ${ }^{12}$ Following broader consultations with stakeholders, providers should agree on terms of reference for the advisory/implementation committee, and invite key stakeholder representatives to participate. Partners should consider, in conjunction with facility management, who will be responsible for coordinating/chairing management meetings with internal and external stakeholders, and who will provide overall supervision for SV services at the facility. 


\section{B. CONDUCT A FACILITY INVENTORYTO ASSESS THE FACILITY'S PHYSICAL CAPACITYTO PROVIDE SERVICES}

Partners in the SGBV Initiative used a standardized facility inventory to assess the readiness of the identified intervention sites to provide services for SV clients at baseline. It was also used as an evaluation tool to measure changes in service quality over the intervention period (comparing endline to baseline.) This tool is included in Appendix 2.

The facility inventory is used to identify the staff, equipment, supplies, and services that are currently available to provide SV care and to identify areas for improvement in the facility infrastructure. The tool covers such issues as: hours of operation, staffing available, location and privacy of examination room, available equipment and supplies, HIV services, availability/use of protocols, patient information materials, referrals and record-keeping. It helps to answer questions such as:

- Does this facility currently provide services to survivors of SV? If so, approximately how many survivors are seen per month?

- What SV services are currently provided in the health facility?

- Where are SV services currently provided?

- How long do survivors generally wait to receive services? Must they wait in the queue or are SV cases given priority?

- How many service providers/departments must the patient interact with during the initial visit?

- Do standardized care and referral guidelines exist?

- Is there a standardized protocol, checklist or kit used for collection of forensic evidence?

- Are the necessary HIV drugs and supplies available in the facility?

- Are other essential equipment and supplies available where SV services are provided?

- Do adequate record-keeping procedures exist for SV cases?

The tool provides a quantitative basis for pre- and post-intervention comparison, as well as comparison across multiple sites, and provides an excellent baseline of information from which to plan the intervention.

TIP: When conducting the facility inventory, take care to assess whether the location, procedures, and availability of staff, supplies and services vary depending on whether the SV patient presents during regular hours, after hours, weekends and public holidays. Partners may find, for example, that SV survivors are only counseled and tested for HIV if they present during regular "office hours," or that patients are examined by specialist doctors during the day but see general casualty doctors at night and on weekends. Understanding these differences and the constraints experienced by the facility at various times is essential for success in the planning process. 
TIP: In addition to completing the facility inventory, it can be very instructive to physically walk through each of the steps that a SV patient would typically follow at the facility from start to finish. As you walk through the process, try to put yourself in the shoes of a SV survivor. At each stage, ask yourself: what aspects of this process are helpful? What aspects might be stressful or frustrating? How does the environment look/feel? Is there adequate privacy? How might this process/setting feel to a woman, a child, a male survivor? How could this experience be improved?

\section{HOLD FOCUS GROUP DISCUSSIONS WITH HEALTH CARE PROVIDERS}

FGDs provide insight into providers' knowledge and attitudes toward SV survivors and the challenges they face in ensuring comprehensive care. In Rwanda and Uganda, FGDs were conducted with doctors and nurses in each intervention site to assess key variables that could impact services. Appendix 3 contains the question guide used in these countries. Other programs have successfully included staff from across the facility (including administrative staff and cleaners) in initial assessments of attitudes.

This qualitative data obtained in a FGD can be very helpful in understanding the context in which services will be provided and the particular challenges faced by providers at a given intervention site. FGDs with providers can help answer questions such as:

- What is the capacity of staff to provide comprehensive SV services, including referrals? Do providers feel they need more training to confidently deliver SV services?

- What are provider attitudes toward SV survivors? Do they affect the type of care that is provided?

- Do providers know where to refer cases for ongoing medical, legal or psychosocial care?

- What are the major perceived challenges to providing SV care at the facility?

- What services and resources are available within and outside the health facility that could support or build on the services provided to SV survivors now?

- What level of support is there at this facility for strengthening SV care?

- How do health providers feel about testifying in court?

The attitudes that providers have about SV survivors are likely to impact the quality of care that survivors receive and are, therefore, especially important to gauge at the outset. Studies from South Africa have found, for example, that some providers do not view SV as a serious health matter or that providers tend to blame the survivors for what has happened to them. ${ }^{13,14}$ These attitudes, and the attitudes of other staff in the facility, can result in lower quality of care and secondary victimization of the survivor. Research has also found that some health care providers are extremely uncomfortable working with traumatized SV patients ${ }^{4}$ or reluctant to conduct exams because they do not want to testify in court. ${ }^{1}$ These attitudes must also be taken into consideration when assessing available staffing and training needs. The focus group process can also be used to obtain more detailed information about services/ treatment provided in the facility and the challenges experienced by those providing care. 
TIP: When conducting FGDs, it is critical to remain aware of power differences among respondents. Doctors and nurses should not be included in the same group due to the common expectation that nurses defer to doctors on clinical issues. This can skew the data by limiting the nurses' ability to respond openly and accurately. If a facility has too few doctors to constitute a FGD (between 5-10 participants), they can be interviewed individually using the same set of questions. Data quality can also be affected by the choice of FGD facilitators, who should be neutral parties. Program staff or supervisors are not recommended data collectors as they may bias participants' responses.

TIP: Any research conducted with human subjects requires their prior consent. The most common method for obtaining consent is through a written informed consent form that is reviewed by the participant and signed prior to conducting the FGD or interview. Once signed, this form should be stored in a secure location and precautions should be taken to ensure that it cannot be used to link individuals to their responses. A sample informed consent form for FGDs is provided in Appendix 4.

TIP: Providers also have private lives, and may have experienced SV themselves or know someone who has. The FGD may trigger negative or traumatic experiences for these providers, and the facilitator must be prepared to address these issues. If possible, it is recommended to have a counselor on site during the administration of the FGD. At the very least, facilitators must be prepared to refer any providers who need further care.

TIP: FGDs are exceptionally useful for conducting formative and baseline research. Using the same tool at endline, however, requires more sophisticated analysis and is often difficult to interpret. A better approach to qualitative endline analysis includes developing a new question guide that explicitly assesses the impact of the program.

\section{IDENTIFY LEGAL REQUIREMENTS FOR PROPER FORENSIC EVIDENCE COLLECTION AND DOCUMENTATION}

The collection and documentation of medico-legal evidence is an important element of a comprehensive response because it is key to the success of prosecution efforts. Proper management of evidence helps to prove SV has occurred and links the suspect to the crime. Forensic management is also essential to allow survivors to access the criminal justice process. In some countries, particularly those with "corroboration"

\footnotetext{
iiii"Corroboration" in the criminal justice context is independent, credible evidence that confirms or corroborates the testimony of the complainant. Statutory corroboration requirements and common law "cautionary rules" are rooted in the historical assumption that the testimony of women and girls in sexual offences cases is inherently suspect and lacks credibility. Although some countries in Africa, such as Kenya and Tanzania, have eliminated corroboration rules and cautions for sexual offences, they remain in force in other countries such as Malawi and Zambia, among others.
} 
requirements, police will not make an arrest for SV unless they have received documentation of injuries or other physical evidence that SV has occurred.

Because of the high reliance by the criminal justice system on medico-legal evidence ${ }^{15}$, partners and providers working in the area of SV must understand the legal requirements and proper procedures for forensic evidence collection and documentation. At the outset of the program, it is important to establish:

\section{THE CHAIN OF EVIDENCE}

"Chain of evidence" refers to the process of obtaining, processing and conveying evidence through accountable tracking mechanisms from the community to the health facility to police. It includes a paper trail where movement of evidence is traceable through the different persons in the chain of sample collection, analysis, investigation, and litigation.

Source: Kenya, Ministry of Health, Division of Reproductive Health. 2009 ( $2^{\text {nd }}$ ed.). National Guidelines on Medical Management of Sexual Violence in Kenya.
- Which medical practitioners (nurses, doctors, clinical officers) are legally empowered to conduct the medical examination/sign police forms/give medical evidence in court?

- What evidence is allowed/used in court for adult and child rape cases that can be collected by medical staff (blood, urine, clothing, hair, fibers, DNA)?

- Are forensic tests possible in country (e.g., DNA, acid phosphatase)? Where are the tests conducted?

- What procedures/protocols/documentation already exists for collecting, storing, and transporting evidence samples? Are these procedures adequate?

- What forms/documentation are required and are admissible in court?

- What are the security/screening requirements, if any, for persons collecting or handling forensic evidence?

- Does the facility have capacity to store forensic evidence for a period of time if the survivor is unsure whether or not to proceed with a criminal case?

- Are survivors required to report to police first to obtain the necessary police form?

- Can/Does the health facility keep spare police forms on hand?

- Are necessary forms being completed properly?

- Who is responsible for collecting the signed form and delivering it to police? (In Kenya, it is the survivor).

- What fees (if any) are survivors being charged for forensic services, laboratory tests or necessary forms?

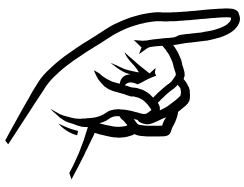

TIP: Some of this information will be available from the health facility or from patient records. Other information may be sought from relevant ministries, such as the Ministry of Justice and national forensic laboratory. Thus, the input of key stakeholders, such as police and SV prosecutors, is essential for gathering reliable and complete information. 
TIP: Program staff should be responsible for compiling this information, as it is central to the design and management of the medico-legal aspects of a SV program. More junior staff from specialized stakeholders, such as police and paralegals, can be effectively engaged to assist in data collection.

\section{E. CONDUCT COMMUNITY-BASED ASSESSMENTS}

Community engagement is critical to the accessibility, use, and sustainability of comprehensive SV services. ${ }^{16}$ Outreach and participatory processes can change the way communities view SV and increase reporting and use of services. Community input can also help to create and streamline referral processes to help survivors access the services they may need in addition to health services: especially legal aid, psychosocial support, economic assistance, or shelter.

Community-based assessments examine the community's understanding of SV and identify barriers and opportunities for accessing SV services. Because attitudes about SV impact the accessibility and use of services, it is important to understand with some specificity how the community views SV and whether and why survivors are stigmatized. It is also critical to understand social barriers to services (lack of information, stigma and blame, lack of confidence in police and other service providers, traditional law practices) as well as economic or physical barriers (distance to services, lack of funds for transport or medical care, economic dependence on the abuser, unavailability of safe shelter). Key questions addressed by community assessments include:

- What are the main concerns and needs of the community with respect to SV?

- How aware are community members of the services offered in the health facility? Do they know when, where and how to seek assistance?

- What are the barriers to seeking care? Do communities report being assessed unofficial charges for services, medico-legal forms or completed documentation?

- How are incidents of SV normally handled by survivors/ communities?

- What community-level resources can be employed to strengthen referrals or care-seeking?

\section{MANAGING THE COMMUNITY ASSESSMENTS}

In the SGBV Initiative, PEPFAR partner organizations generally led the project's community engagement activities, rather than the clinics and hospitals themselves. Those partners who were able to hire a dedicated manager to lead the process tended to have the best results. The Initiative supported the local partners by providing short-term technical assistance in the form of tools, training workshops, and site visits to build the capacity of the partners to engage communities, identify resources and needs, and plan activities.

Under the SGBV Initiative, community assessments took the form of FGDs that were undertaken in some communities. Unlike the provider FGDs, which were conducted by external facilitators, health care providers were trained to conduct these FGDs within their own communities. This allowed them to both collect necessary data for program planning and increase their personal knowledge of community needs. ${ }^{17}$ Appendix 6 includes the community FGD guide used under the Initiative. 


\title{
STEP 2: ESTABLISHING THE REGULATORY AND INFRASTRUCTURAL FRAMEWORK FOR SERVICES
}

\author{
When should this be done? Prior to launching program. \\ What staff are needed? \\ Many of the activities in this step require policy decisions that \\ must be made with the involvement of national officials, facility \\ and program managers. \\ Other resources required: Some basic infrastructural investment may be required to ensure \\ that services are co-located to the extent possible, and visual and \\ auditory privacy is offered. Additional supplies and equipment \\ may need to be procured for the service delivery point, and \\ adequate stationery is required for record-keeping.
}

Using the findings of the initial assessments outlined in Step 1, programs can begin to ensure that the regulatory and physical framework is in place for delivering quality SV services.

\section{A. ENSURE THAT FACILITY-LEVEL GUIDELINES FOR SV SERVICE DELIVERY ARE IN PLACE}

Every health facility should have a detailed clinical protocol in place which describes the way all SV cases should be managed and which contains specific information and guidance for health providers at each step in the process, from the initial intake to referrals and follow-up care. The purpose of these protocols is to help facilities provide consistent, high quality treatment and support to SV survivors based on recognized standards. Protocols help guide health providers step by step through the complex and often lengthy procedures required for comprehensive care and medico-legal examination, and provide a valuable tool for training, monitoring and evaluation, and quality control.

Basic elements of a SV care protocol include procedures for:

- Survivor intake and crisis counseling

- Documenting medical history 
- Obtaining consent

- Performing a thorough medico-legal examination

- Collecting forensic evidence

- Performing diagnostic tests and prescribing treatments

- Administering VCT and PEP

- Recording findings for use in treatment and court (including the proper completion of police and other national reporting forms)

- Providing follow-up care and referrals

The management protocol also may contain additional sub-protocols, such as for emergency contraception, PEP, and treatment/prevention of STIs, as well as special protocols for child survivors. It can provide additional instructions for providers on collection of patient data for monitoring and evaluation purposes, and/or coordination with other stakeholders such as police or social services.

A number of countries have taken steps to develop national health protocols or guidelines for management of sexual assault, including Ethiopia, Kenya, Malawi, South Africa, Rwanda, Uganda and Zambia. In some cases, national guidelines have not been widely disseminated and may not be well known or used by practitioners at the facility level. Thus it is important to first establish whether national guidelines for clinical management or multi-sectoral management of sexual violence have been developed or are in place in the country where you plan to work. If national guidelines are available, these should provide the basis for services at the site and serve as the starting point for training and local protocol development. ${ }^{\text {iv }}$ If national guidelines do not exist, partners should engage government at all levels to develop national guidelines and interim protocols for care at the intervention site. Protocols should follow WHO guidelines as much as possible, with appropriate adaptations for the particular country or facility. ${ }^{10}$ National guidelines from other African countries can also be instructive and useful (see the "helpful resources" section).

When developing clinical protocols, it may be helpful to obtain input from members of the community as well as health providers. Community input can help to ensure that services are culturally appropriate and meet the needs of survivors as defined by the community. This process may follow somewhat naturally from community-based assessments if the assessment methodologies encourage community members to be critical in identifying barriers and opportunities, and to identify and advocate for priorities for improving clinical response to SV. ${ }^{18}$ For example, information from communities may help the health facility determine whether to provide weekly supplies of PEP or the full 28 days of PEP at the outset, depending on the ability of survivors to return to the site for follow-up care. Assessments may also tell you whether survivors prefer to access services outside their immediate community (due to fears of stigmatization) or whether services would be better utilized if closer to home.

\footnotetext{
ivIn South Africa's Refentse program, the hospital established an official Hospital Rape Management Policy, based on the national guidelines, which laid out treatment protocols addressing specific problems identified during the assessment stage. These included 24-hour access to VCT, introduction of an early STAT dose of PEP, and dispensing a full 28-day course of PEP on the initial visit. See Kim, J., et al. 2009. "The Refentse Model for post-rape care: strengthening sexual assault care and HIV post-exposure prophylaxis in a district hospital in rural South Africa." Washington, DC: Population Council.
} 


\section{B. MINIMIZE MULTIPLE REFERRAL POINTS}

One of the most important changes partners can make in their intervention is to help facilities streamline services and reduce points of contact for SV survivors in the hospital or health center. ${ }^{4,6}$ Careful attention should be given to the location of these services. Factors such as privacy, accessibility (especially during nights and weekends), staffing, comfort and security (including separation from suspects) must be taken into account.

Various models exist for streamlining services, and many require minimal investment apart from procedural changes. In some facilities in Malawi, Uganda, Kenya and South Africa, this has been done by locating all medico-legal or SV services in one unit of the hospital and actively referring patients to other outside services (such as counseling and criminal justice services). ${ }^{3}$ In South Africa's Refentse model, the hospital established a dedicated, unmarked room for SV counseling and examinations in the outpatient department (OPD). All tests and treatments were centralized and available in the designated room, eliminating the need for patients to queue up at the pharmacy or obtain VCT and PEP from the ART clinic. Integration of services in the round-the-clock OPD also ensured that all tests and treatments, including VCT, EC, and PEP, were available to survivors 7 days a week, 24 hours per day. An expanded role for nurses in the delivery of SV care, and clear management protocols, also contributed to a reduction in the number of service points. Similar approaches were implemented by SGBV initiative partners, such as Mulago Hospital in Uganda. There, 24-hour SV services were located in the gynecology department, with referrals made to in-hospital police services and outside psychosocial care providers. ${ }^{3}$ Four other facilities in Uganda also established dedicated rooms where none previously existed. ${ }^{5}$

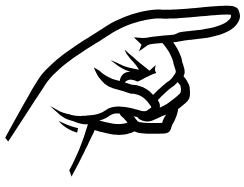

TIP: Community assessments may be particularly useful in informing the identification of a location for services. For example, partners may find that survivors prefer to receive services in or near the general outpatient department rather than in the ART clinic, because of stigma associated with HIVIAIDS. Communities may also be willing to assist with services, e.g., by providing donations of spare clothes, toys or food for SV survivors.

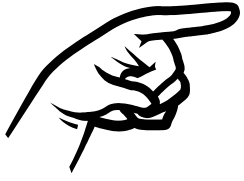

TIP: Since the majority of rape cases present after-hours and on weekends, it is important to locate SV services, whenever possible, in or near a department that is open 24 hours per day. In many cases, facilities may choose to locate SV services in a unit that does not provide 24-hour care. To ensure that survivors can receive care during off-hours, alternate SV service provision points can be established in areas that do not close, such as the OPD or maternity wards.

Reducing anxiety and ensuring survivor privacy and confidentiality is also a critical component of quality SV care. To this end, it is important to create a quiet, dedicated space in the facility where survivors can be counseled and examined in privacy, without the risk of others overhearing. ${ }^{9}$ If possible, a separate waiting area should also be provided so that traumatized patients and young children do not have to wait in a crowded, stressful environment such as casualty or OPD, or risk coming into contact with a perpetrator. In the Refentse project, an existing room in the OPD was made more private with a few minor changes. 
TIP: Once a room is identified, consider whether simple renovations can be made to make the room more pleasant and child-friendly. Paint, posters, blankets, pillows, and toys are relatively inexpensive, but can assist children as well as adults to feel more relaxed and comfortable in the examination environment. If possible, keep simple food on hand (tea, biscuits), as the process may be long and the survivor may not have eaten in several hours. Having food may also reduce nausea from EC and PEP medications.

TIP: Once a location for SV services is established, both internal and external stakeholders (facility staff, police, other health providers) should be informed about the SV services available and where SV services will be provided. To minimize waiting time, SV cases should be given priority in the queue. This procedure should be established as an official policy of the health facility and front desk officers/information desk personnel and police informed of the new procedures. Develop and place posters or signs in the reception area that explain what SV services are available in the facility, the cost of obtaining the services and what patients/ caregivers should do to obtain expedited assistance.

\section{ENSURE THAT SV EXAMINATION POINTS ARE STOCKED WITH NECESSARY SUPPLIES, EQUIPMENT AND GUIDELINES}

Appendix 7 provides a list of basic equipment, medications, and supplies needed for SV cases, and facility-specific protocols should contain a more detailed list of the supplies required for SV services. The accessibility of these supplies can be verified by the facility inventory. All supplies necessary for comprehensive care should be available in or near the designated examination room(s), 24 hours per day. Special care should be taken to ensure that sufficient quantities of pediatric medications (i.e., PEP syrups) and special examination tools for child survivors are available if required.

TIP: Before setting up the SV service, partners should work with the facility managers to identify and, if necessary, re-allocate the supplies and equipment available in the health facility. In the SGBV Initiative, partners often found that equipment (such as speculums or examination lamps), supplies (swabs, tubes, HIV test kits) or medications (such as sedatives, EC and PEP) were available in the facility, though not in the departments where SV services were normally provided. Sometimes equipment or supplies were found in storerooms, not being used. Where essential items are unavailable for SV care, partners should encourage (and if necessary, assist) providers to motivate management and/or the Ministry of Health to purchase necessary equipment/supplies/medications for use in the dedicated SV room, and include recurring items in the regular budget for the facility. Including stakeholders from management and/or relevant ministries in the project advisory committee can facilitate this process. Purchase of equipment and supplies with outside funding should be limited to unique items if possible. 
TIP: Rather than purchasing expensive, pre-packaged rape kits, facilities in low-resource settings can assemble their own materials or "kits" for evidence collection from supplies routinely available at district hospitals. These may include, for example: gloves, swabs, large sheets of paper, envelopes, paper bags, tape, and specimen containers or tubes. ${ }^{19}$

All relevant protocols, forms (including consent forms), and tools such as flow charts, check-lists, patient information materials and referral lists should also be readily available in the SV examination room. Some providers find it very useful to post algorithms/flow charts and other information on the walls, or to integrate the protocols into their clinical forms for ease of reference and use. In one South African site where providers were having difficulty prescribing correct PEP dosages for children, the hospital pharmacist developed a simple pediatric PEP dosage chart, which was posted in the examination room. ${ }^{4}$ In the Refentse model, nurses developed a medications counseling form which included pictures of the various medications typically prescribed to SV survivors (EC, antibiotics, anti-emetics, PEP), instructions for taking the medication, and information about side effects to aid the providers in adherence counseling. The form was later used by researchers to assess patients' understanding and adherence to the medication's regimen. ${ }^{6}$

TIP: Patients who have recently been traumatized by SV frequently have difficulty absorbing or remembering the large amount of complex information provided to them during the initial visit. It is therefore helpful to provide patients and/or family members with written information to take home, to explain and reinforce the information provided at the health center. These should include, at minimum, information about the health consequences of SV (including emotional and psychological effects), treatment prescribed (including medication and side effects), and follow-up. Information materials should be simple, inexpensive to reproduce, and available in local languages whenever possible (see Appendix 8 for a brochure used in Kenya). If the patient is prescribed PEP, it is helpful to provide detailed written instructions explaining the purpose of PEP, the regimen to follow, the importance of completing the full course, and information on coping with side-effects.

\section{ENSURE ADEQUATE RECORD-KEEPING PROCEDURES ARE IN PLACE FOR SV CASES}

Establishing adequate record-keeping systems is essential for monitoring the quality of SV services, improving follow-up care and ensuring that medico-legal documentation is available for prosecution. Most health information systems in Africa, however, do not specifically capture information on SV clients and medico-legal documentation is undermined by forms that lack necessary detail.

To address this, partners in the SGBV Initiative introduced a comprehensive SV client form to document the care and treatment provided to survivors across all intervention sites (see Appendix 5). The form was adapted for the project from the Kenyan Ministry of Health Post-Rape Care (PRC) Form, the South African J88 form, and the "SV Examination Record" template contained in the WHO Guidelines for 
Medico-legal Care for Victims of Sexual Violence, 2003. The purposes of the form were to: a) compile data for routine program monitoring; b) guide the examination process to ensure all services offered; $c$ ) provide detailed reference documentation for doctors who may be called to court; $d$ ) track referrals and follow-up care. Many providers, however, found the forms too long and time-consuming to fully complete for each SV client. Missing data most frequently involved information on services that were provided in other hospital departments, such as HIV testing and PEP provision, and follow-up care. Thus, when developing a record-keeping system it is important to balance the need for information with realistic expectations on the provider's time.

TIP: Adequate record-keeping is a core challenge of many health systems which is compounded by the introduction of detailed medico-legal forms. Because of the importance of medicolegal documentation in prosecution, reducing the information required on the forms is not recommended. Instead, efforts should be made to ensure that all cadres of providers (including nurses) are trained to complete the forms. In the SGBV Initiative, nurses were more likely than doctors to provide complete documentation.

TIP: It is especially important in SV cases to establish security procedures and systems to protect patient confidentiality and control access to survivor files. (Medical files containing information relevant to a criminal case may be particularly vulnerable to theft or tampering). Some guidelines recommend maintaining an independent filing system for SV patients. If using a general filing system, at minimum consider keeping copies of the key SV documents (medical examination records, consent forms, and the police form) in a separate locked file for added protection against loss.

\section{E. REDUCE OR ELIMINATE FEES FOR MEDICAL AND MEDICO-LEGAL SERVICES}

Fees for medical services and/or medico-legal examination can serve as a barrier to SV care in low-income settings. Determine whether there is a national law or policy to provide free medical services to victims of rape and sexual assault and, if so, whether this law/policy is implemented consistently at the facility. In the absence of a national policy, try to negotiate free services with the hospital/clinic management. Managers often have discretion to waive fees in certain circumstances, including at referral hospitals. In Zambia, for example, hospitals acted to remove "grey fees" for victims of SV (e.g., fees to complete and sign medical forms) by posting signs at the reception area identifying that all SV services are free of charge. This change helped ensure that the national policy of not charging fees to SV survivors was consistently implemented in the hospitals associated with the study. ${ }^{12}$ Also in Zambia, the A Safer Zambia (ASAZA) program worked with the management of Livingstone Hospital to waive fees for treatment of domestic violence victims. ${ }^{20}$ 


\section{STEP 3:TRAINING HEALTH CARE PROVIDERS}

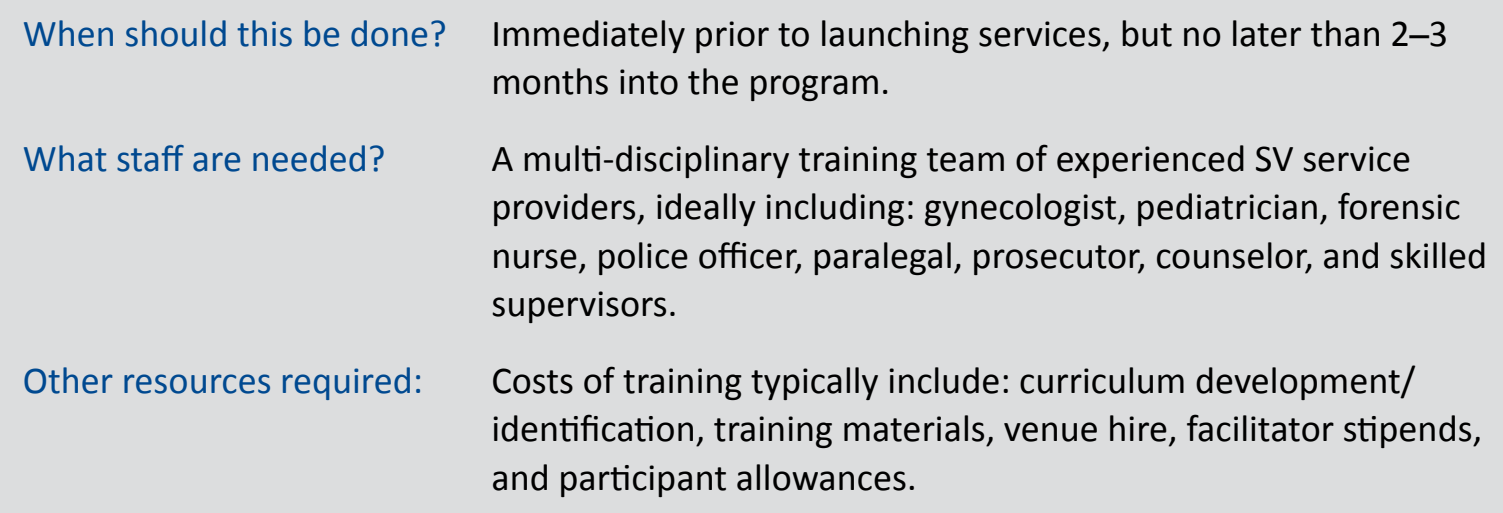

What staff are needed? A multi-disciplinary training team of experienced SV service providers, ideally including: gynecologist, pediatrician, forensic nurse, police officer, paralegal, prosecutor, counselor, and skilled supervisors.

Other resources required: Costs of training typically include: curriculum development/ identification, training materials, venue hire, facilitator stipends, and participant allowances.

Training of health care providers is an essential step in ensuring access to quality SV services. In most African countries, doctors, nurses and clinical officers do not routinely receive pre-service training on SV services and do not feel adequately prepared to offer care to survivors. The majority of providers participating in the SGBV Initiative indicated at baseline that they had not received sufficient training on SV care, and demonstrated limited knowledge of the essentials of case management. ${ }^{5}$ To improve quality of care and ensure adequate implementation of the protocols and procedures established in Step 2, it is necessary to train SV service providers. As a multi-sectoral effort, this training should include counselors, police, paralegals and other partners assisting in the delivery of SV services.

\section{A. CORE ELEMENTS TO BE INCLUDED IN SVTRAINING PROGRAMS}

To the extent possible, training of health care providers and other stakeholders should utilize adult education methods such as participatory learning, critical reflection/discussion, small group work, case studies, demonstrations, and role plays, in addition to presentations by the instructors. This methodology helps to build skills and empower participants to think through issues and challenges, rather than just impart information. Trainers should also seek to create a safe environment in which participants can reflect on their own attitudes and experiences and participate actively in the learning process.

The initial training of providers can range from a one to two-day workshop to a two-week comprehensive training, depending on the needs identified at the intervention site and the availability of resources.

Refresher trainings can then be scheduled based on provider needs and turnover. Provider training should cover the following issues: ${ }^{\mathrm{v}}$

vAdapted from Jina, R. et al. 2008. "Caring for survivors of sexual assault and rape: A training programme for health care providers in South Africa." Pretoria: South African Department of Health. 


\section{Social context of GBV, and sensitization to the issues}

- Examination of personal attitudes and values relating to gender, violence, and SV

- SV as a public health concern; the importance of comprehensive care

- Definitions, circumstances, context of rape and sexual assault; social norms and attitudes/links between gender inequality and vulnerability to sexual and gender-based violence

- The experience of the survivor; barriers to reporting; health and social consequences of sexual violence; secondary victimization

\section{Legal framework}

- Sexual offences under national law (rape and defilement)

- Legal obligations of health care providers (including mandatory reporting of child abuse, when applicable)

- Required legal documentation

\section{Initial approach to rape survivors (preparing the survivor to receive services)}

- Ethical considerations in delivering SV care

- Basic counseling and trauma debriefing/containment skills

- Communication skills for initial consultation and history taking

- Taking a history and obtaining consent

- Special considerations for children, male survivors, and people with mental disability

\section{Managing clinical conditions and conducting examinations}

- Prevention and management of pregnancy (including the provision of emergency contraceptives)

- Prevention and management of sexually transmitted infections

- Prevention and management of HIV (including providing VCT, PEP and referrals to ART, if required)

- Providing PEP to children

\section{Conducting medico-legal examinations and documenting injuries}

- Types and use of forensic evidence

- Genital and non-genital injuries

- Examination of children and interpretation of findings

- Special considerations for examination of elderly, physically or mentally challenged, and pregnant survivors, male survivors and perpetrators 
- Collection of forensic evidence in SV cases

- Documentation of medical examination, findings and conclusions; including use of the standard police form(s)

- Protection of evidence/chain of custody

- Introduction to the legal system and giving of expert testimony

\section{Referrals and follow up to the initial consultation}

- The importance of providing referrals to ensure comprehensive care

- Developing and utilizing referral directories and tools

- Recommended follow-up schedule, services provided at each visit

- Strategies for promoting PEP adherence

\section{Monitoring and evaluation of post-rape care}

- Data collection and quality assurance methods

\section{Mentoring and supervision (caring for the carers)}

- Vicarious trauma and counter transference

- Mentoring, debriefing and supportive supervision for health care providers.

Examination of children, PEP administration for children, and documentation of evidence are frequently cited as areas of concern by providers, and should be specifically emphasized in the training curriculum. If possible, the training should also include a practical component, with mentoring by experienced health practitioners.

TIP: Training on comprehensive SV services is best provided by a multi-disciplinary training team. Police and legal sector representatives are best-positioned to speak about the law and the uses of evidence in prosecution. Psychologists or professional counselors can provide an additional dimension to discussions on psychosocial care and vicarious trauma. Medical specialists, such as gynecologists, pediatricians and forensic nurses can provide more detailed instruction on clinical care issues.

\section{B. IDENTIFYTHE SERVICE PROVIDERS TO BE TRAINED, DEPARTMENTS TO BE INCLUDED IN SV SERVICE PROVISION}

Partners should work with facility management to identify the health providers and other personnel to be trained to provide SV services. This will depend in large part on which departments (e.g., OPD, casualty, 
OB-GYN, pediatrics, medico-legal, etc.) will be primarily responsible for SV management and care, and whether care will be provided by designated staff/specialists or any staff member on duty at a given site. Selection for training should take staffing patterns into account so that there is at least one trained SV service provider on duty or on-call at the relevant department 24 hours per day. As the vast majority of survivors are female, facilities should also ensure that female doctors, nurses, and/or clinical officers are included in the training.

To the extent possible, it is recommended that the health facility identify and train dedicated practitioners (doctors, forensic nurses, and/or clinical officers) to conduct the medico-legal examinations in SV cases, particularly those involving young children. It is also helpful to include hospital managers, lab technicians, pharmacy staff and communications specialists in relevant sections of the training.

TIP: If it is not feasible to provide dedicated staff for SV services, the facility should maintain a roster of trained SV providers to examine and treat survivors in the dedicated area on an oncall basis. At least one designated nurse, counselor or volunteer should be trained and available 24 hours per day to provide crisis and HIV counseling to survivors in the designated area.

TIP: Nurses and/or clinical officers can play a much more expansive role in the provision of SV services than is currently provided at most sites. Nurse-driven services reduce the amount of time that survivors must wait before being attended to, and are necessary, particularly in rural areas, where few doctors are available to provide SV care. ${ }^{3}$

Supervisors of SV providers should also be included in the training to familiarize themselves with the elements of comprehensive care, clinical protocols, and the how to provide supportive supervision and regular debriefing for SV providers. Each facility should have a supervisor responsible for monitoring the SV services and providers at that site.

TIP: Supervisors should be trained to provide supportive supervision and regular debriefing support to SV providers in their facility or department. Supervision may be individual or in groups. Working with rape survivors is complex and emotionally taxing. Ongoing mentoring and support is critical to monitor quality of service, build skills, and provide emotional and professional support to practitioners. ${ }^{21}$

At minimum, all health facility staff working with sexual violence patients (including doctors, nurses, pharmacists, HIV counselors, intake clerks, orderlies, etc.) should undergo basic sensitization training and orientation to the SV clinical guidelines and referral protocols developed under the program. All should have a good understanding of the elements of comprehensive SV management and how to approach and communicate with survivors to minimize secondary trauma. They should also become familiar with the facility's intake, follow-up, and referral procedures/resources for SV patients. 


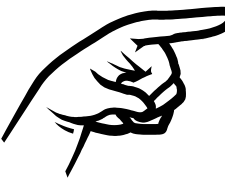

TIP: If the facility has high turnover or frequent rotations of staff, the basic training/orientation should be repeated at regular intervals. To maximize the value of training, it is also advisable to agree with facility management on how long a provider should work in the area of SV after training, not only to gain experience but also to bear in mind that frequent changes of staff do disrupt provision of services adversely. Management should arrange for smooth handover of service provision and mentoring of replacements by the trained provider in situations where staff movement is inevitable.

TIP: A lesson learned from the SGBV Initiative is that partners and facilities should try to identify health providers who have a particular interest or "passion" for working with SV survivors, and who have good communication and counseling skills, to receive specialized training in SV management. These providers should be supported and mentored to develop their skills, and given opportunities and incentives to specialize in this area of work. ${ }^{5}$

\section{CONDUCT CROSS-TRAINING WITH REFERRAL PROVIDERS, INCLUDING THE POLICE AND LEGAL SECTORS}

In addition to (or as part of) providing specialized training for health care providers, it is very helpful to organize a shorter one to two-day multi-disciplinary training for all key stakeholders involved in the management of rape and sexual assault cases. This may include, for example, police and prosecutors responsible for the investigation and prosecution of rape cases; judicial officers presiding on SV cases and their court personnel; social workers and other providers responsible for addressing the patient's social and mental health needs; HIV-AIDS counselors; and non-governmental or community-based organizations (CBOs) involved in providing crisis counseling and/or follow on support to rape survivors.

In addition to sensitizing SV partners across sectors, cross-training is helpful for exploring the roles and responsibilities of each of the relevant stakeholders and how the response of each role player affects that of others. For example, it is important for police officers to understand that long delays at the police station can contribute to the patient's trauma and undermine the effectiveness of EC and PEP. Likewise, health care providers must understand how delays in completing the medical reports can compromise arrests. This approach has been used successfully in Zambia to increase collaboration between the sectors and improve referrals. ${ }^{12}$ 


\title{
STEP 4: INCREASING PEP ADHERENCE AND FOLLOW-UP CARE
}

\begin{abstract}
When should this be done? Providers must be trained to deliver counseling on adherence and follow-up care during the initial visit. Barriers to seeking and providing follow-up care should be identified during the initial assessments (Step I) and strategies to overcome them can be developed and refined throughout implementation.

What staff are needed? Most follow-up care is provided by the same health care providers who deliver SV services. Some specialized strategies, such as providing telephone reminders or conducting home visits, require additional non-clinical staff.

Other resources required: A full course of PEP, anti-emetics and client informational material are basic requirements for each survivor that presents. Other follow-up services can be provided based on available resources.
\end{abstract}

Follow-up appointments are recommended to monitor the survivor's condition and treatment, assess her/his mental and emotional state, provide follow-up counseling and support, and conduct follow-up tests for HIV. Further referrals may also be advised during follow-up visits to the site. The schedule for follow-up will depend on national guidelines, facility protocols and the needs of the individual survivor. WHO guidelines recommend follow-ups at two weeks and six months for survivors not on PEP. ${ }^{8}$ In South Africa, follow-up appointments are recommended at three days or one week, one month, six weeks and three months. ${ }^{9}$ If PEP is prescribed, some facilities may require more frequent follow-ups to obtain additional medication and monitor adherence and side effects. The South African Department of Health also recommends weekly follow-ups during the first six weeks to provide regular psychological support. ${ }^{9,22}$

TIP: Many survivors do not access follow-up care. This is often attributed to limited resources, stigma within the community or the effects of post-traumatic stress disorder.To ensure the highest possible follow-up rate, programs must consider these barriers at inception and identify community-specific strategies for overcoming them. These approaches are likely to require refinement throughout implementation. 


\section{A.ADDRESS PEP ADHERENCE ISSUES THROUGHOUT PROJECT PLANNING AND IMPLEMENTATION}

PEP adherence was consistently identified as a major challenge by service providers participating in the SGBV Initiative. ${ }^{4,5,20}$ Although adherence rates are difficult to estimate because of the high loss to followup rates, available data from South Africa finds that PEP completion ranges from 20 to 58 percent of all clients who initiate PEP. ${ }^{3}$ A study conducted for the Gauteng Department of Health in South Africa identified the following factors as contributing to low PEP adherence: ${ }^{14}$

- Patients did not understand how to take the medication, either because it was not explained properly or the patients were too traumatized to take in and remember the information

- Patients were not treated for side effects (nausea and vomiting)

- Patients could not afford transport for return visits to test and/or obtain additional PEP

- Patients were not provided support to complete the regimen

- Attitude of health workers (judging, questioning whether patient is lying about rape to get PEP, etc.)

The following strategies have been identified to improve PEP adherence in low-resource settings and should be considered from the project planning stage.

\section{Minimize the number of return visits required to obtain a complete course of PEP}

Clinical programs should avoid instituting systems in which the patient must return multiple times for additional PEP, as transport and economic barriers often prevent patients from returning to the site. 4,6 Rather, partners should take steps at the initial visit to ensure that patients understand how to take the medications correctly and receive support and treatment for side effects.

If possible, health providers working with SV patients should be trained to provide VCT, so that counseling is not limited to when regular HIV counselors are available. Rapid tests are readily available and should be used at SV sites so that patients are not required to return for test results before receiving their medication. In the SGBV Initiative, nurses from several units at Mulago hospital were trained to provide VCT, PEP and EC, so that these services could be made available for SV survivors on a 24-hour basis. ${ }^{5}$

"Starter packs," which include the initial 3-5 days of PEP, usually provided because VCT is unavailable, are not recommended for provision in health settings because of low follow-up rates. In the Refentse project in South Africa, only 14 percent of those provided with a starter pack returned to the site for additional medication. ${ }^{6}$ Rather, efforts should be made to improve the availability of VCT and HIV services at all times in health facilities. Where VCT is not available at the initial visit (or the patient refuses testing), patients should be given a starter-pack, followed by the remaining PEP if the patient tests negative for HIV at the next visit. 


\section{Prescribe anti-emetics to control nausea and vomiting, and provide adherence counseling and written information}

Anti-emetics should be provided at the initial visit concurrent with the PEP with instructions to take if needed for nausea. ${ }^{6,89}$ This reduces the likelihood that the patient will stop taking the medication due to side effects, and eliminates the need to return to the facility to obtain a second prescription.

Effective counseling has also been shown to increase PEP adherence. ${ }^{23}$ The counselor should take time during the initial visit to explain the purpose of PEP and the treatment regimen, and counsel the survivor about side effects and the need to complete the full course of treatment. Roles and responsibilities for counseling on PEP should be clearly defined in the site protocol.

Many traumatized patients and caregivers experience information "overload" during their initial visit and have difficulty remembering the PEP regimen and why it is important to complete the full course and return for follow-up tests. Information booklets-ideally in the local language of the survivorcan assist in reinforcing the information obtained at the initial visit and can also be used by caregivers, families and friends to support the survivor.

\section{Use the simplest regimen available}

A 2007 study in South Africa found a wide range of practices in the administration of PEP, depending how the medications were packaged, from 2 pills per day $/ 4$ per week (Combivir) to 8 per day $/ 56$ per week. ${ }^{4}$ Fifty-six sites supplying larger numbers of pills reported more problems with patients skipping pills (particularly azidothymidine [AZT]) than those providing simpler regimens. National Guidelines in Kenya specifically recommend the use of "fixed dose combinations" for SV, if available as "this reduces the number of pills taken and so increases compliance to treatment."23

\section{B. IMPLEMENT STRATEGIESTO IMPROVE ACCESS TO FOLLOW-UP CARE}

Follow-up is an important step in SV care, but also one of the most challenging. In low-resource settings it is often difficult - for a variety of reasons-for survivors to return to the health facility for follow-up care. It is therefore important to:

- Ensure that follow-up arrangements and referrals are in place before the survivor leaves the facility;

- Provide maximum input during the first visit, as it may be the only visit to the site. ${ }^{10}$

Specific strategies for increasing SV survivors' utilization of follow-up care include the following.

\section{Issue appointment cards}

Many health facilities routinely provide the patient with an appointment card noting the dates of follow-up appointments. ${ }^{4}$ This may help survivors and caregivers remember appointments, but is unlikely to be sufficient on its own without additional support, where other barriers to follow-up exist. Care must also be taken to ensure that providing a card does not compromise the survivor's privacy or safety, particularly where an abuser is in the same home. 


\section{Provide telephone reminders}

To implement this kind of system, a staff member will need to track patients and contact those with an upcoming appointment to remind and encourage them to return for follow-up. Alternate phone numbers (friend, family member, work number) for the survivor should be obtained at intake in case the patient's phone number changes, and facilities must establish and follow confidentiality protocols for these calls to avoid compromising the survivor's privacy or safety.

\section{Combine follow-up appointments with other services}

A review of health facilities in South Africa found that those with the highest rate of return for follow up are those that "make visits relevant to the needs of survivors, and particularly those that more adequately address their mental health needs." ${ }^{22}$ Some

\section{TELEPHONE REMINDERS}

Nurses at the Pediatric Center of Excellence at Livingstone Hospital in Zambia call caregivers a few days before each scheduled appointment to remind them of follow-up appointments and to explain the importance of returning for follow-up care. Providers have reported that this contributes to higher return rates.

Source: USAID/Zambia. 2010. Evaluation of gender-based violence programming. programs in Africa have reportedly found it effective to coordinate clinical follow-ups at the health facility with other services, such as psychosocial counseling or support groups offered on-site or in the same general area, or a meeting with the victim assistant officer or paralegal to discuss the status of the patient's case. Combining services helps to control transport costs and may also act as an incentive for the patient to return for clinical follow-up. ${ }^{4}$ Other service providers (such as counselors and support groups) can also play a role, where appropriate, in reinforcing messages regarding the importance of PEP adherence and follow-up testing, for example.

\section{Provide transportation assistance}

In low-resource settings, transportation is probably the most significant practical obstacle to receiving follow-up care. ${ }^{1,12}$ Patients using public facilities tend to come from lower-income groups, and many lack transport or funds to return to the facility after the initial visit. Thus, transport barriers are commonly cited by clients as a primary reason for not seeking follow-up care. A variety of innovative approaches have been undertaken by partners to minimize this barrier.

- In Uganda, SGBV Initiative partners learned of a local NGO that would provide transportation for survivors to the health facility, including follow-up appointments. The NGO established a telephone "helpline" and provided emergency transport as one of their services. Where resources are available, a few SV services have opted to provide transport vouchers or reimbursement for transport costs to patients returning for follow-up.

- In South Africa, the Thohoyandou Victim Empowerment Programme (TVEP) initiated a transport assistance scheme in cooperation with a local bus company to address transport challenges in a large rural area. Under this scheme, the bus company donates bus tickets to the TVEP center, which the center then distributes (two at a time) to survivors to allow them to return home and then return to the center for their next follow-up. The tickets can also be used by TVEP staff to conduct home visits to the patients in their communities. 
- A few isolated programs have also accessed hospital "petty cash" (or funds from the staffs own pockets) to provide small amounts to individual patients for transport.

- Some programs have relied on program or police vehicles to provide transport in some cases, although the sustainability and effectiveness of this strategy has not been established. ${ }^{20}$

\section{Refer to local clinics or ART sites for follow-up HIV testing}

Survivors who live a long distance from the health facility and are unable to return to the site can be referred for follow-up HIV tests closer to their homes. Follow-up testing is recommended for survivors who take PEP as well as those who present after the 72 hour cutoff. If possible, the referring facility should have a system in place to follow-up these results, either with the survivor or directly with the clinic.

\section{Offer follow-up services through mobile clinics}

Mobile clinics can bring a variety of services to the communities where survivors live, including VCT, STI treatment, family planning, psychosocial and paralegal counseling. They can also be used to raise awareness about SV and services at the health facility. Some SV programs such as ASAZA, Greater Nelspruit Rape Intervention Programme (GRIP) and Liverpool VCT care and Treatment (LVCT) periodically go out to communities to offer free counseling and advice to SV survivors. GRIP also partners with other PEPFAR partners to provide VCT and information about PEP in the context of SV. To protect confidentiality, SV services are best provided in conjunction with general health and/or HIV counseling and information.

\section{Conduct home visits where possible}

Two South African NGOs, TVEP and GRIP, effectively utilize a "case manager" approach with SV clients. Under this system, the same counselor who conducts the intake of the survivor when he/she first presents follows that survivor's case from beginning to end. This includes visiting the survivor's home within the first week after the initial visit to the site to check on the survivor's status, and providing emotional support, information, and food packets where needed. Additional home visits may be conducted, particularly if the survivor is taking PEP or requests supportive intervention with her family. 


\section{STEP 5: DEVELOPING REFERRAL LINKAGESTO OTHER SERVICE PROVIDERS}

When should this be done? Referral linkages should be identified during program planning or in the initial phases of implementation, and regularly updated as new resources are available in the community.

What staff are needed? Ideally, health care providers would conduct the community mapping and develop referral strategies. Realistically, it may be necessary to recruit partners or community groups to carry out the work under the guidance of the program manger.

Other resources required: Limited resources are required for these activities, including transportation to map referral points, mobile phone credit, and per diems and refreshments for participants. Some costs may be associated with implementing new referral systems, such as stationery and printing.

In most cases — even in "one-stop" settings ${ }^{\mathrm{vi}}$ — providing comprehensive care to SV survivors will require one or more referrals to other service providers located outside of the health facility. These providers can be any government office, NGO (including faith-based organizations [FBOs] and CBOs), or individuals who provide direct services to SV survivors and their families, including police, prosecutors, social services, community shelters and safe havens, legal advice centers, local clinics, women's organizations, and organizations providing psychosocial care. Ensuring strong referral linkages to and from these providers is a critical, yet often underdeveloped element of clinical SV services. ${ }^{18}$

TIP: Several factors influence the potential strength of referral linkages in a given community. These can include proximity of services to one another, attitudes of service providers, levels of awareness of services in the community, use of standardized referral algorithms, and ongoing meetings between stakeholders.

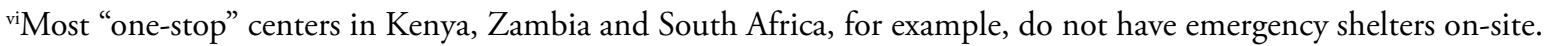
All provide some counseling at the initial visit, but many must refer to outside organizations for specialized services for children and/or longer-term counseling and social support.
} 


\section{A. CONDUCT PARTICIPATORY COMMUNITY MAPPING OF ALL REFERRAL POINTS}

The first step is to identify service providers in the community to whom and from whom SV survivors can be referred for services. This can be conducted in conjunction with the community FGDs discussed in Step 1. Under the SGBV Initiative, health care providers were coached to conduct participatory community mapping exercises with communities. Community mapping can also be conducted by project partners or community-based organizations to reduce the demands placed on health care providers.

"Community mapping" is a process wherein community members are invited to construct a visual representation of a community, including the physical, economic and social infrastructure. The community map makes it possible to generate discussions on diverse resources in a given community and can sometimes bring to light key community and political structures, economic resources, and gaps within this infrastructure for which solutions can be offered. The mapping exercise also helps assess the accessibility of existing referral resources, highlights areas of potential collaboration among partners, and identifies other complementary projects that may be funded by PEPFAR that can be leveraged.

\section{B. DEVELOP A FORMAL REFERRAL DIRECTORY AND PROTOCOL FOR OUT- REFERRALS AND POST INTHE SV EXAMINATION ROOM}

Based on the information gathered from the community mapping process, the health facility can then develop a formal referral directory for SV services provided elsewhere. The key steps for compiling such a list include: $:^{\text {vii }}$

1. Call or visit each institution identified in the community mapping to gather up-to-date information about services, location, populations served, hours of operation, fees, etc., and determine whether and how the institution can be used for referrals. It is generally best to do this in person to meet the service providers and view the services firsthand.

2. Organize the information in a directory (by location, by type of service) or a list, if the number of referrals is small.

3. Distribute the directory to health providers who work with SV survivors and keep a copy readily available in the room used for SV counseling and/or examination. If the list of referrals is small, post it on the wall of the counseling or examination room.

4. Formalize relationships with referral institutions. This may include setting up formal referral and counter-referral systems (as well as systems to track referrals), agreeing on standards for confidentiality/ service delivery/data collection, and/or collaborating more closely on projects and activities. Referral organizations can also be invited to provide services (such as counseling or taking of police statements) to survivors on site at the health facility.

viiAdapted from Bott S. et al. 2004. Improving the Health Sector Response to Gender-based Violence: A Resource Manual for Health Care Professionals in Developing Countries. London: International Planned Parenthood Federation. 


\section{COORDINATING REFERRALS}

In Zambia's ASAZA program, each Coordinated Response Center (CRC) has established an advisory council and service provider network to improve coordination, planning and problem-solving with community stakeholders and other service providers. At Thuthuzela Care Centres (TCCs), representatives from the police, prosecutor's office and NGO partners participate in monthly implementation meetings organized by the site coordinator.
5. Organizations in the referral network should be in contact with each other on a regular basis to give feedback, and provide follow-up on cases and other issues related to the work. If possible, include key referral organizations in the program's quality assurance and M\&E plan. Key referral organizations can also be asked to participate in regular stakeholder/project implementation meetings and multi-sectoral training.

6. Update the information in the directory on a regular basis and gather feedback from providers (and survivors) on how well the referrals are working.

\section{DEVELOP AN ALGORITHM OF CARE (FLOW DIAGRAM) THAT INCLUDES EXTERNAL REFERRALS}

Each facility should develop a simple flow diagram which illustrates the key steps in the SV treatment protocol, and the order in which the steps take place. The steps should be similar across sites (intake, containment, history taking, examination, etc.), but the order may vary somewhat from site to site. The flow diagram should include the scheduling of follow-up appointments and referrals to external service providers. If multi-sectoral services are available on-site at the facility (i.e., a social worker or police officer to open the case) these services should also be included in the algorithm. Flow diagrams are useful tools for service providers, as they help envision how services are organized, and how the client progresses from step to step. Appendix 9 provides an example of an algorithm of care developed by Mulago Hospital in Kampala Uganda, which includes internal and external referrals.

\section{CONSIDER DEVELOPING REFERRAL SYSTEMS TO FACILITATE AND TRACK USE OF REFERRAL SERVICES}

Consistent tracking of out-referrals is a challenge for many SV programs. Without some kind of system in place, however, it is difficult to know whether survivors are receiving follow-up services such as ART, counseling or other psychosocial support. Some health facilities and NGOs provide survivors with a letter or referral card to keep and take with them to the referred service provider(s). In addition to contact details, the card includes a space for the service provider to indicate the dates on which the survivor presented and received services. This card is reviewed each time the survivor returns to the facility or NGO for follow-up appointments and the information captured in the survivor register or file. These systems are not always successful, however, as they depend on the survivors to bring the cards back. In certain situations, having a SV referral card in his or her possession may also jeopardize the survivor's confidentiality or put the survivor at greater risk. These issues should be considered before instituting a card-based referral system. 


\section{STEP 6: STRENGTHENING LINKAGES BETWEENTHE HEALTH FACILITY AND COMMUNITIESTO INCREASE TIMELY UTILIZATION OF SERVICES}

\begin{abstract}
When should this be done? Programs should engage with the community from the outset, although community-wide awareness strategies are best conducted after SV services are functional.

What staff are needed? Health care providers or other non-clinical program staff can conduct community outreach activities. Technical assistance from communications partners may be required to develop and implement effective messaging.

Other resources required: The development of new communications materials and purchasing radio time can be costly. Adapting existing materials is less expensive, and some community media will donate time to NGOs or CBOs.
\end{abstract}

To ensure the utilization of the services available within the health facility, it is critical that communities are aware that the services exist and how to access them. Essential messaging includes the need for survivors to seek SV health services as soon as possible, no later than 72 hours of the assault.

TIP: Programs should allow sufficient time to implement and evaluate outreach activities, as awareness and attitude change is a long-term process requiring commitment, planning, and ongoing intervention.

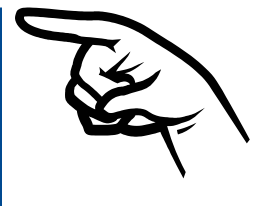




\section{A. IDENTIFY COMMUNITY LEADERS AND PARTNERS}

Before developing a community engagement strategy (and during the project planning and budgeting process) partners and stakeholders should consider who will be responsible for leading community information and outreach activities and whether outside technical support or training is needed. In the SGBV Initiative, PEPFAR partner organizations generally led the project's community engagement activities, rather than the clinics and hospitals themselves. Those partners who were able to hire a "champion", or individual from the community who actively led the process, tended to have the best results.

\section{B. DEVELOP COMMUNICATION MATERIALS FOR COMMUNITY AND CLINIC-BASED AWARENESS CREATION}

Posters, pamphlets, and other written materials on SV and SV services can be useful for conveying information and reinforcing messages, if culturally relevant and written in a language understood by members of the community. Under the SGBV Initiative, PEPFAR partners collaborated with the Ugandan NGO Raising Voices to develop and validate a set of posters to raise awareness about what SV is and how to access services if someone experiences it.

The posters used the principle of positive or benefits-based messaging, and were reviewed and adapted for use by partners in Uganda and Rwanda. One poster was designed to be displayed in the community: it identifies what $\mathrm{SV}$ is and states that $\mathrm{SV}$ is not tolerated in that community (see Appendix 10). Another two posters were intended for use in a clinical setting: they emphasize the availability of services and encourage survivors to get treatment within 72 hours of abuse (see Appendix 11).

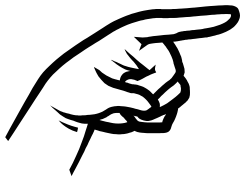

TIP: In general, IEC materials should be simple and written in a language understood by most people in the area. In limited-resource areas, materials that can be copied easily and at low cost are generally preferable to glossy pamphlets requiring color or specialized printing services. When developing IEC materials, it is important to carefully consider the target audiences and adapt the materials and mediums accordingly. In general, IEC messages should be simple and targeted, i.e., "Emergency contraception can prevent pregnancy if used within 5 days of rape or other unwanted sex.Visit your local clinic for assistance. Don't delay!"

TIP: In all outreach communications, it is important to employ positive role modeling to foster a sense of community identity and nonviolence, emphasizing community support for nonviolence and empathy for survivors rather than sensationalizing violence against women or portraying men as evil and dangerous. In the PEPFAR SGBV Initiative, partners requested and participated in workshops that taught them how to raise awareness about SV in the community, and how to design communications materials using this positive messaging approach. 


\section{INTEGRATE SV MESSAGES INTO EXISTING HEALTH SERVICE OUTREACH ACTIVITIES}

Many health facilities have existing outreach programs that disseminate information about health and health services. Such programs pose excellent opportunities to raise awareness about SV and its health consequences, as well as available services for survivors. As part of their clinical services, partners should look for opportunities to integrate SV messages into existing HIV, maternal and child health, family planning and other reproductive health programs. ${ }^{24}$

Partners can also design campaigns specifically focused on SV issues, such as sexual coercion among young people, child sexual abuse, and rape. Messages should focus on the health consequences of sexual violence (including, but not limited to, HIV infection), the availability of services to prevent HIV infection and pregnancy from sexual violence, how and where to access services, and the urgency of seeking medical care within 72 hours after rape.

In the PEPFAR SGBV Initiative, several partners incorporated SV into their health education programs. In Rwanda, the International Center for AIDS Care and Treatment Programs (ICAP) created sessions on SV services in its "morning sessions," wherein nurses deliver health-related talks to patients in the clinic waiting room. The Northern Uganda Malaria, Tuberculosis and HIV AIDS Program (NUMAT) in Uganda added information about SV and its root causes - as well as information about health services for survivors - in its peer education curriculum. Other programs have also successfully integrated SV messages into existing outreach, peer education, and community care programs. In Zambia, for example, ASAZA partners trained existing community health workers on SV, integrated SV into the training and resource materials, and added a module on SV to youth peer education programs. ${ }^{20}$ In many countries, Peace Corps volunteers are involved at grassroots level in conducting health and HIV-related education and outreach, and could also be trained and supported to integrate SV messages into their health-related activities.

\section{CREATE CLINIC-COMMUNITY LINKAGES TO SENSITIZE COMMUNITIES AND INCREASE SERVICE UTILIZATION}

If possible, partners are encouraged to adopt a multi-pronged approach to community awareness, combining information about services with messages to sensitize the community about the issue of SV. Several partners in both Rwanda and Uganda advertised their services on radio. The Rwandan partners are in the process of developing a common radio message that all partners can use to advertise services. Peer education and small group discussions were also used by Initiative partners to raise awareness about SV services and how to access them. Discussions included information about where to go for help, services offered and time limitations for receiving PEP and emergency contraception.

Initiative partners also created links between clinical services and other SV services by identifying other services in the community, building relationships with these institutions, and creating referral networks to increase survivors' access to the full range of available services. In Rwanda, Drew Cares and AIDS Relief hosted stakeholder meetings in their communities; and ICAP held more informal meetings with the police to garner their support for referring survivors to clinical services. In Uganda, the Mulago-Mbarara Teaching Hospitals' Joint AIDS Program (MJAP) brought together community-based service providers based in the Kampala area for the first time: this meeting resulted in a collaborative effort to draft referral 
protocols. NUMAT (Uganda) hosted monthly coordination meetings in most of its project communities with stakeholders, maintaining an ongoing commitment to multi-sectoral SV response. Such meetings created and strengthened critical links between clinical services and the community that will likely increase survivors' access to the range of services they need.

During the endline evaluation of the SGBV Initiative, community members, stakeholders, service providers and program managers in Uganda identified the community sensitization activities as one of the most successful aspects of the SGBV initiative. Participants reported that community outreach had raised awareness, changed attitudes in the communities, increased the numbers reporting within 72 hours, and was even believed to have reduced the number of SV cases within the community. ${ }^{5}$ 


\section{STEP 7: MONITORING AND EVALUATING THE PROGRAM}

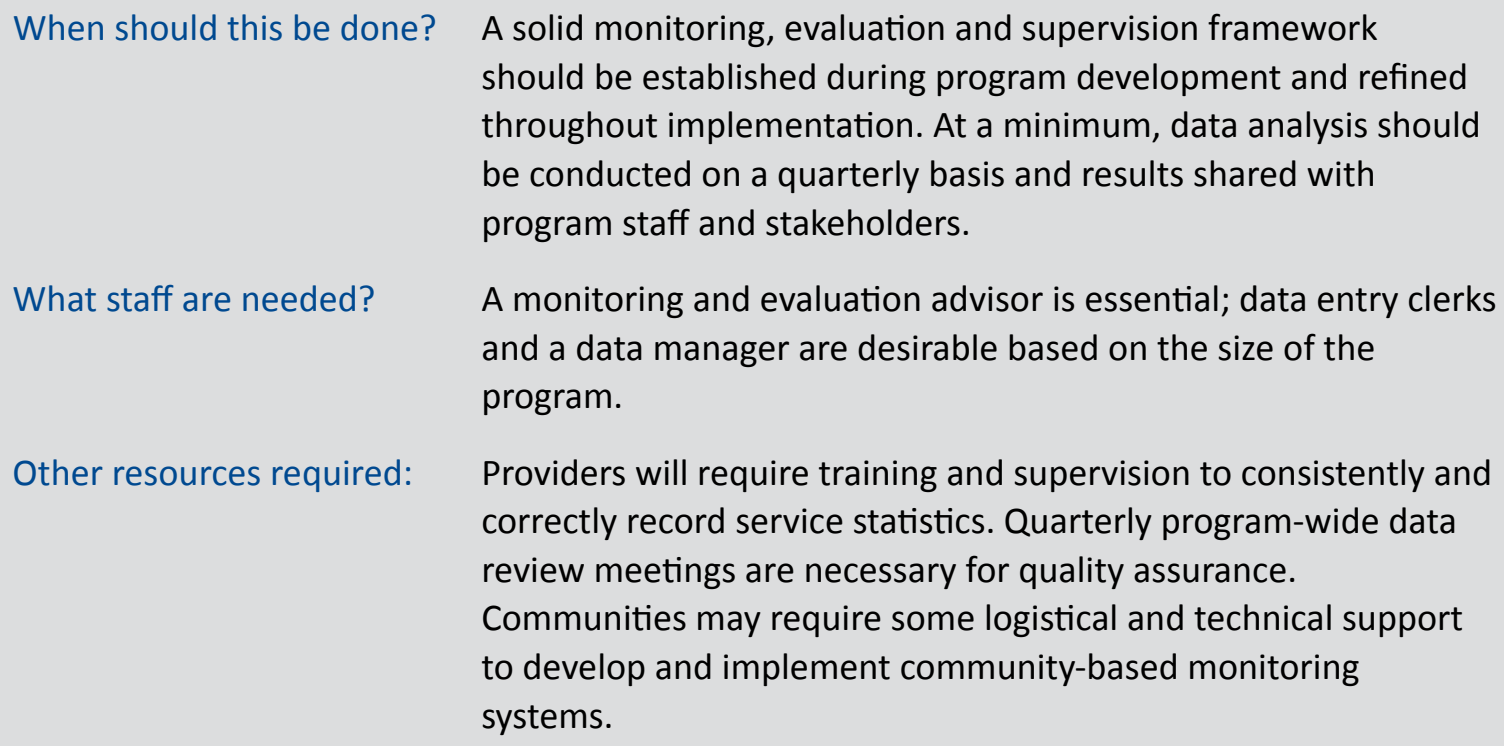

When should this be done? A solid monitoring, evaluation and supervision framework should be established during program development and refined throughout implementation. At a minimum, data analysis should be conducted on a quarterly basis and results shared with program staff and stakeholders.

What staff are needed? A monitoring and evaluation advisor is essential; data entry clerks and a data manager are desirable based on the size of the program.

Other resources required: Providers will require training and supervision to consistently and correctly record service statistics. Quarterly program-wide data review meetings are necessary for quality assurance.

Communities may require some logistical and technical support to develop and implement community-based monitoring systems.

Routine monitoring and evaluation $(\mathrm{M} \& \mathrm{E})$ of $S V$ services is essential for ensuring that the program is meeting its objectives of providing quality, accessible care to survivors of SV. It is also essential for identifying good practices and lessons learned in providing comprehensive SV care that other programs can learn from. Limited evidence exists on the most effective strategies for providing SV services in low-resource settings, and reliable program data is important for building the evidence base for future programming.

\section{A. IDENTIFY APPROPRIATE INDICATORS}

The first step in developing an effective M\&E strategy is identifying appropriate and meaningful indicators. Two of the PEFPAR "Next Generation" indicators are specifically intended to measure the effectiveness of SV interventions. ${ }^{25}$

- P6.1.D: Number of persons provided with PEP, by exposure type (occupational, rape/sexual assault victims, or other non-occupational).

- P6.2.N: Percentage of health facilities with HIV PEP available, by exposure type (occupational, nonoccupational). 
- P12.2.D: Number of people reached by an individual, small group or community-level intervention or service that explicitly addressed gender-based violence and coercion related to HIV/AIDS.

In addition, a broader set of SV-related indicators has been developed and tested by partners working in the area. This measurement framework, developed by the Population Council, includes a set of validated indicators for measuring the outputs, outcomes and quality of comprehensive SV services (Table 2). ${ }^{3}$

Table 2:Validated indicators for measuring comprehensive SV service programs

\begin{tabular}{|c|c|c|}
\hline Sector & Indicator & Data Source \\
\hline \multirow{5}{*}{$\begin{array}{l}\text { Client } \\
\text { characteristics }\end{array}$} & Number and percent of survivors presenting to site by month & Health facility records \\
\hline & Number and percent of survivors by age, sex, gender and month & Health facility records \\
\hline & $\begin{array}{l}\text { Number and percentage of: } \\
\text { - Adult rape cases (male and female) } \\
\text { - Child rape cases } \\
\text { - Intimate partner violence cases (IPV) }\end{array}$ & Health facility records \\
\hline & $\begin{array}{l}\text { Percent of survivors reporting to: } \\
\text { - Police station first } \\
\text { - Health center/ hospital first }\end{array}$ & Health facility records \\
\hline & $\begin{array}{l}\text { Mean and median time elapsed (in hours) from assault to: } \\
\text { - Care seeking at health center } \\
\text { - Reporting to police station }\end{array}$ & Health facility records \\
\hline \multirow[t]{6}{*}{ Health services } & $\begin{array}{l}\text { Percent of facilities that have a dedicated room available for examining SV } \\
\text { survivors }\end{array}$ & Facility inventory \\
\hline & $\begin{array}{l}\text { Mean and median number of health providers seen by clients during the first } \\
\text { visit }\end{array}$ & Health facility records \\
\hline & Percent of survivors given trauma counseling & Health facility records \\
\hline & $\begin{array}{l}\text { Percent of eligible survivors receiving core services at health facility (eligibility } \\
\text { defined by national guidelines), including: } \\
\text { - Percent of eligible clients given emergency contraception (at health facility) } \\
\text { - Percent of eligible clients given STI prophylaxis } \\
\text { - Percent of eligible clients given tetanus prophylaxis }\end{array}$ & Health facility records \\
\hline & $\begin{array}{l}\text { Percent of survivors receiving core HIV services } \\
\text { - Percentage of eligible clients who receive HIV test results during first visit } \\
\text { - Percent of eligible clients given first or "stat" dose of HIV PEP } \\
\text { - Number of ARV/PEP pills given to survivor during first visit } \\
\text { - Percent of PEP recipients referred for adherence counseling } \\
\text { - Percent of PEP recipients presenting for follow up care at I week; } 6 \text { weeks }\end{array}$ & Health facility records \\
\hline & $\begin{array}{l}\text { Mean and median number of weeks survivors presented for their first follow-up } \\
\text { visit }\end{array}$ & Health facility records \\
\hline
\end{tabular}




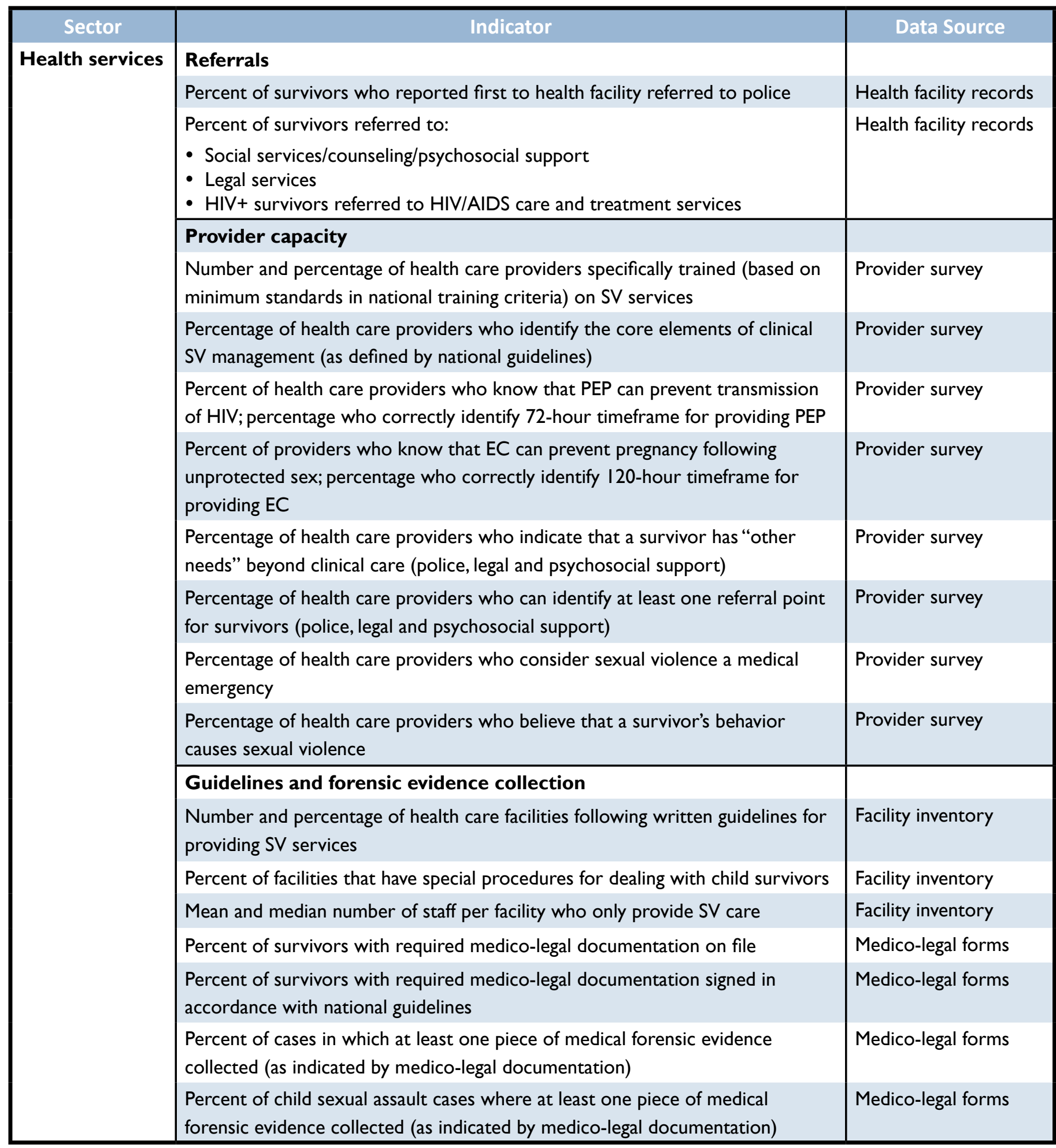




\begin{tabular}{|c|c|c|}
\hline Sector & Indicator & Data Source \\
\hline \multirow{16}{*}{$\begin{array}{l}\text { Police/legal } \\
\text { services }\end{array}$} & Percent of survivors who report to police station within 120 hours after assault & Police records \\
\hline & $\begin{array}{l}\text { Percent of survivors eligible for emergency contraception } \\
\text { - denominator }=\text { total number of survivors presenting at facility of } \\
\text { reproductive age } \\
\text { - numerator }=\text { total number of survivors presenting within } 120 \text { hours }\end{array}$ & Police records \\
\hline & $\begin{array}{l}\text { Percent of eligible survivors receiving emergency contraception from police } \\
\text { station }\end{array}$ & EC program records \\
\hline & $\begin{array}{l}\text { Percent of survivors referred to: } \\
\text { - Health facility } \\
\text { - Social services/ counseling/ psychosocial support } \\
\text { - Legal services }\end{array}$ & Police records \\
\hline & Provider capacity & \\
\hline & $\begin{array}{l}\text { Number and percentage of police facilities following written guidelines for } \\
\text { responding to SV services }\end{array}$ & Provider survey \\
\hline & $\begin{array}{l}\text { Number and percentage of police officers specifically trained (based on } \\
\text { minimum standards in national training criteria) on SV services }\end{array}$ & Provider survey \\
\hline & $\begin{array}{l}\text { Percent of police officers who know that PEP can prevent transmission of HIV; } \\
\text { percentage who correctly identify } 72 \text {-hour timeframe for providing PEP }\end{array}$ & Provider survey \\
\hline & $\begin{array}{l}\text { Percent of providers who know that EC can prevent pregnancy following } \\
\text { unprotected sex; percentage who correctly identify } 120 \text {-hour timeframe for } \\
\text { providing EC }\end{array}$ & Provider survey \\
\hline & Percentage of police who consider sexual violence a medical emergency & Provider survey \\
\hline & $\begin{array}{l}\text { Percentage of police who believe that a survivor's behavior causes sexual } \\
\text { violence }\end{array}$ & Provider survey \\
\hline & Legal outcomes & \\
\hline & Percent of cases where a suspect was identified & Police records \\
\hline & Percent of cases with charges filed & Court records \\
\hline & Percent of cases with a conviction & Court records \\
\hline & Mean and median number of years sentenced & Court records \\
\hline \multirow{3}{*}{$\begin{array}{l}\text { Psychosocial } \\
\text { support } \\
\text { services }\end{array}$} & Number of sites offering psychosocial care in catchment area & Community mapping \\
\hline & Number of survivors seeking psychosocial support, disaggregated by type & Service records \\
\hline & $\begin{array}{l}\text { Mean and median number of psychosocial support sessions/meetings attended } \\
\text { by each survivor }\end{array}$ & Service records \\
\hline
\end{tabular}

\section{B. IDENTIFY APPROPRIATE DATA COLLECTION/CAPTURE TOOLS}

Table 2 indicates a variety of data sources for each of the indicators. This list includes many of the tools discussed in the previous sections which can be both for ongoing program monitoring and final program evaluations. These include: 
- Facility Inventory (Step 1B and Appendix 2): In addition to setting a baseline for improving services, this inventory can be conducted at regular intervals to assess the program's progress. In the SGBV Initiative, it was conducted at baseline (pre-intervention) and endline (post-intervention), and results were compared between the two points in time. For longer programs, the inventory can also be conducted at mid-point to identify potential program improvements.

- SV Client Form (Step 2D and Appendix 5): Client records can provide highly useful data on client characteristics, the types of services provided, referral practices and follow-up care. However, these data present inherent challenges associated with the completeness and accuracy of records and require frequent data quality checks. (See Step 2D for a discussion of the difficulties associated with collecting client data.) Nonetheless, these data are exceptionally valuable for monitoring the program's effectiveness and can be analyzed over time and by facility to identify performance trends. Data on client characteristics can also comment on the effectiveness of community outreach activities.

TIP: The medico-legal form should be completed during the history taking and examination process to record the patient history, injuries, specimens collected, tests, treatment, and referrals. The form helps structure the examination and ensure that all necessary steps are completed. Health providers should be discouraged from completing the form after the fact from unstructured clinical notes - and particularly the notes of other providers-as this is a time-consuming process which may also result in incomplete or incorrect documentation.

TIP: In addition to review by M\&E staff, medical supervisors should also periodically review a sample of completed medico-legal forms to assess the quality and consistency of documentation. This process helps supervisors identify gaps or weaknesses in services as well as the completion of forms, and take necessary steps to address them.

- FGDs with providers and program managers (Step 1C and Appendix 3): Qualitative data can provide additional insights into the program's effectiveness and perceived impact. While baselineendline comparisons of qualitative data are often challenging, FGDs or key informant interviews (KIIs) can be conducted at various points throughout the intervention to identify strengths and weaknesses in the intervention and to identify strategies for overcoming them. At endline, FGDs are useful for documenting the perceptions of those involved in implementing the program, especially in terms of the program content.

\section{CREATE COMMUNITY MONITORING SYSTEMS}

Providers are also encouraged to institute systems for ongoing feedback from the community about how well the services are meeting the community's needs. In the PEPFAR SGBV Initiative, partners were trained to use participatory approaches to encourage community input into the process of monitoring the provision of SV services. HPI also developed a questionnaire for focus groups to solicit feedback on how well service providers were meeting the challenge of responding effectively to SV and what community members perceived to be the most pressing actions yet to be taken. ${ }^{18}$ 
TIP: Direct observation of client-provider interaction, the use of "mystery clients," and FGDs are NOT recommended for evaluating SV services, due to their sensitive nature. Some programs solicit feedback on service satisfaction from survivors who participate in 'survivors' groups or other psychosocial support services. Informed consent is required for all such interviews with survivors, and interviewers must be prepared to refer survivors for support if the interview induces further trauma.

\section{ONGOING QUALITY ASSURANCE AND SUPERVISION}

Ongoing assessment, monitoring and supervision is essential to ensure that services provided to $\mathrm{SV}$ survivors meet the standards established by national guidelines and/or local protocols. Supportive supervision and facilitated assessment and action planning also creates an environment conducive to problem-solving and professional development.

LVCT Care and Treatment in Kenya employs various methods to monitor and improve quality of SV services at its sites: These include: ${ }^{\text {viii }}$

- Indirect observation (review of records, supportive supervision, regular debriefing sessions for staff)

- Data collected from program staff and partners (self assessment check-lists from service providers, feedback from partners and other service providers)

- Routine analysis of service data

- Ongoing needs-assessment and training

In the Refentse program, the research nurse reviewed patient files and interviewed consenting SV survivors during follow-up appointments to assess the nature and quality of services received, adherence to medical regimens (including PEP), and client perception of services provided. Site visits and structured interviews with service providers were also used by the Thuthuzela Care Centre program to assess compliance with national Department of Health standards for sexual assault care.

TIP: Providers who work with SV survivors need an outlet to talk about the cases they see, in order to reduce emotional stress and potential for burnout. Starting support groups for providers is a low-cost way to create this outlet, while giving providers a chance to discuss problematic professional issues that they encounter in working with victims of gender-based violence. Case conferences and facilitated debriefings are more structured approaches to supervision and support, recommended where resources permit. ${ }^{26}$

\footnotetext{
viiiSee http://www.liverpoolvct.org.
} 


\title{
STEP 8: ENSURING ADEQUATE RESOURCES
}

\author{
When should this be done? Careful budgeting of both start-up and recurrent costs should \\ take place before the program is implemented. \\ What staff are needed? Program mangers, governments and donors must be involved in \\ budgetary planning and negotiation.
}

Planning for SV programs also entails budgeting appropriately for start-up costs, developing a sustainability strategy for recurrent costs, and planning for scale-up from inception.

\section{A. MOBILIZE RESOURCES FOR ONE-TIME PROGRAM START-UP COSTS}

The cost of establishing or strengthening SV services at an existing health facility will vary according to the setting, extent of training required, and level of equipment and services already available. It will also depend on whether the service can be provided within the existing facility as is, or whether additions and/ or refurbishment are needed to meet minimum standards

(e.g., a private examination room).

Costs to be considered include start-up one-time costs and recurrent costs. For sustainability, recurrent costs (particularly those associated with the operational costs of the facility and provision of medical care) should be assumed by the facility and built into the budget from the outset. Start-up costs are most likely to require external support.

The start-up period for implementation may extend over a long period of time before the full range of services is available at a given site. Start-up for SGBV Initiative partners in Rwanda and Uganda ranged from two months to two years, and included needs assessment, training, engaging with local authorities, establishing stakeholder groups, and community sensitization.

Typical start-up costs include:

- Any remodeling of facilities needed to provide necessary privacy, such as a private counseling room and/or examination room/waiting area dedicated to the provision

COSTING THE REFENTSE PROGRAM In the Refentse program, the total cost of strengthening the existing post-rape service at Tintswalo Hospital in rural South Africa was $\$ 84,612$ over three years, including facility-level costs-capital investment, training and materials development, and salaries of a SV coordinator and research nurse- and patient level costs associated with medication use, tests and referrals. The estimated cost for delivering the intervention was approximately $\$ 200$ per case.

Once the one-off materials development costs and initial costs of the nurse and coordinator were excluded, the incremental intervention costs decreased to $\$ 58$ per case.

Source: Kim, J. et al. 2009. “The Refentse Model for postrape care: Strengthening sexual assault care and HIV post-exposure prophylaxis in a district hospital in rural South Africa. Washington, DC: Population Council. 
of post-rape services; refurbishments (furniture, painting, addition of bathing facility or kitchen, etc.) to create a more survivor-friendly/child-friendly space

- Assessments and focus groups (health provider and community)

- Adaptation of tools and clinical protocols

- Training of health providers/multi-sectoral training workshops (venue, materials, trainers)

- Technical assistance (planning, adaptation of protocols and tools, quality assurance)

- Equipment and supplies for basic sexual and reproductive health services (if not already available), lockable cabinets for files and evidence

- Staff time to set up appropriate linkages and referral systems with police, judiciary, psychosocial services, and other support services in community

- Possible creation or upgrading of laboratory facilities for analysis of biological and forensic evidence in SV cases

- Initial community awareness and outreach activities (i.e., awareness campaign, launch event), development and production of IEC materials in appropriate languages

- Development/adaptation of M\&E system and instruments, equipment needed for data collection (i.e., computer), training on use of tools

\section{B. ENSURE AVAILABILITY OF RESOURCES FOR ONGOING RECURRENT COSTS}

It is recommended that partners work with the facility and other key stakeholders from the outset of the project to develop a sustainability plan to support recurrent costs after the project ends. It may also be possible to share costs with other organizations in the area working on SV issues, or for partner organizations to take over responsibility for stakeholder coordination, outreach, and monitoring/ evaluation over time.

Recurrent costs typically include:

- Fixed operational costs of the facility/SV service

- Supplies, medications, laboratory tests, additional staff (these costs may increase over time with increased uptake of services)

- Ongoing training and mentoring of health personnel

- Debriefing support (care for carers)

- Production and dissemination of IEC materials

- Ongoing awareness-raising and behavior-change activities

- Periodic self-assessment and action planning

- Stakeholder meetings and coordination

- SV service or project coordinator 


\section{START-UP AND RECURRENT COSTS UNDERTHE SGBV INITIATIVE}

According to SGBV Initiative partners, main costs at start-up included the cost of providing a full or parttime program manager for the project, training of service providers and community leaders, and community sensitization.

- In Uganda, start-up activities at the largest site included multi-sectoral training for 60 health providers (four 3-day trainings), police and social workers, refurbishment of facility infrastructure (to add space for an office and laboratory), purchase or requisition of supplies and equipment, and establishment of a data management system. The partner also hired a full-time SV services supervisor to oversee program implementation.

- One partner in Rwanda reported that it spent approximately $\$ 10,000$ to conduct three 5-day training programs, two for health care providers and one for police and local leaders.

- Another partner also provided two training sessions for health providers, as well as one training of trainers at central level with the $\mathrm{MOH}$.

- A Ugandan partner identified high costs for training of supervisors in SV management as well as specialized training in forensic evidence collection for doctors and nursing officers in the SV unit.

Most PEPFAR partners in Rwanda and Uganda found it necessary to support the health facilities with equipment, medications and other supplies in order to start the program.

- In Rwanda, one partner reported that it spent approximately $\$ 2,000$ on various equipment and supplies, but this amount was not sufficient to provide all the materials and equipment needed. The partner noted in particular the need for basic medications, such as EC, as well as materials for collecting forensic evidence which were not available at the intervention site.

- Another partner agreed to provide materials for evidence collection, but reported that other supplies were also needed at the facility - in particular, EC pills and drugs for STI prevention, cameras for documenting injuries, and extra clothing for survivors. These items were not included in the project budget and have not been provided to the facility by the $\mathrm{MOH}$.

- In Uganda, one partner spent approximately $\$ 6,700$ at start for supplies and equipment for seven intervention sites. This included such items as speculums, gloves, examination lamps and lockable cabinets. Another $\$ 4,466$ in medications and supplies were obtained from other departments, such as the ART clinic and family planning unit. As in Rwanda, this partner reported that this amount was insufficient to start-up quality SV services, and that additional resources were needed at its SV sites for basic equipment such as examination beds, photocopying of police forms, and transport for survivors.

Among the SGBV Initiative partners, recurrent resource requirements most frequently included:

- The cost of a full or part-time program manager, as well as staff or volunteers to plan and implement community awareness activities.

- Each facility required a permanent staff of health care providers, available 24 hours per day to provide SV services.

- Several partners also reported recurring costs for drugs and other supplies. This ranged from approximately $\$ 1,500$ per month total for seven sites in Uganda to approximately $\$ 5,000$ per month (including about $\$ 3,000$ per month for laboratory reagents) for one partner in Rwanda. 


\section{CONCLUSION: PROMOTING COUNTRY OWNERSHIP, SUSTAINABILITY AND SCALE-UP}

All of the strategies outlined in this guide are intended to promote the establishment of sustainable and cost-effective SV services through collaboration with key stakeholders from across the sectors. Most often, this collaboration takes place at the facility-level. However, programs must also actively engage with national-level stakeholders to create the policy environment needed to sustain and scale-up SV services.

\section{A. ENSURING COUNTRY OWNERSHIP AND SUSTAINABILITY}

Country ownership is essential for success at all stages of the program. At the outset, governments must recognize the need for improving SV services and see it as an appropriate area for investment. During the program planning phase, they must provide the leadership and vision for the national SV program through the development of necessary guidelines and policy frameworks. This may include, for example, establishing national clinical or multi-sectoral guidelines and authorizing an expanded role for nurses and clinical officers in providing medico-legal services. Throughout implementation, government buy-in is important for ensuring that programs have the necessary technical and financial support to succeed.

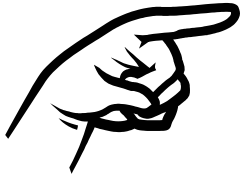

TIP: High-level buy-in can help overcome many of the challenges associated with human and material resource constraints that are often beyond the authority of facility managers.

The multi-sectoral nature of comprehensive SV services requires that a range of Ministries are included in program planning and implementation. In many countries this includes the Ministries of Health, Justice, Home Affairs (Police), Gender, and Community Services. While these stakeholders may work together at the community-level, they are less likely to actively collaborate centrally. To promote such coordination, many countries have formed national-level multi-sectoral working groups to guide the implementation of SV programs. 
TIP: While multi-sectoral working groups are useful, they may be difficult to coordinate because of the competing demands and priorities of the Ministries involved. To address this, in some countries the leadership of the multi-sectoral group rotates on an annual basis; in others, the Ministry most involved in providing SV services assumes permanent leadership. The strength and authority of the Ministry appointed to head the multi-sectoral group should be considered at time of establishment, as a weak Ministry is likely to experience difficulty in coordinating more powerful partners.

Within each participating Ministry, it is important to have designated persons or "focal points" to coordinate SV services at both national and district level. Particularly when scaling up services, a focal person in the Ministry can help provide leadership and assist partners in overcoming institutional problems and challenges. In Kenya, for example, a coordinator for post-rape care services was appointed from the Ministry of Health's (MOH) District Management Team in each district where integrated services were established. National leadership and oversight responsibility rested with the Reproductive Health Division of the Ministry of Health. ${ }^{27}$

In some programs, sustainability can also be encouraged through partnership with a strong, local NGO. Throughout implementation, the capacity of these NGOs can be built to manage the program, coordinate stakeholders, and provide technical assistance and support to the service providers. In South Africa, NGOs such as GRIP, TVEP, Tshwaranang and Mosaic are working effectively in partnership with public health facilities and other stakeholders to provide comprehensive SV services in their communities.

\section{B. CONSIDERATIONS FOR TAKING PILOT SV PROGRAMS TO SCALE}

Planning for scale-up should be included in initial program development activities. At the conclusion of the SGBV Initiative, health providers and community stakeholders felt strongly that the SV programs started under the Initiative should continue, and that efforts should be made to scale-up the program across the two countries. ${ }^{5}$ To do this, programs must have the explicit support of the government and key stakeholders to guide and manage the process.

TIP: Particular attention should be given to expanding SV services at lower-level facilities such as clinics and health care centers, to increase access to services and reduce obstacles caused by lack of transport.

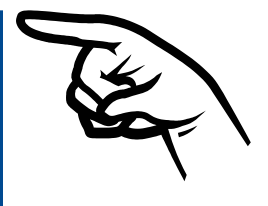

All stakeholders, especially government, should be involved in planning for scale-up. Some questions to guide the process include ${ }^{\mathrm{ix}}$ :

ixAdapted from "Worksheets for Developing a Scaling-up Strategy" June 2010 (draft). ExpandNet and World Health Organization. http://www.expandnet.net/tools.htm 
- Does sufficient evidence exist to demonstrate that the program works and is cost-effective?

- Is SV considered to be a problem in the areas where the services are to be scaled up? If not, do awareness-raising activities need to be conducted prior to introducing services?

- What additional resources will be required at the national, provincial and facility level to provide comprehensive SV services? Are those resources available?

- Do national-level guidelines on comprehensive service provision exist to guide the scale-up process? Is the policy framework strong enough to support effective prosecution efforts?

- Is external technical assistance required? If so, who will provide that assistance and how will it be funded?

- At what type and level of facility will comprehensive SV services be provided? Will they be located within hospitals, health facilities, or at other sites?

- Will the program be scaled-up across the country at one time, or will introduction be phased?

- What individuals and Ministries will be responsible for ensuring that quality, comprehensive services are provided? What oversight will be provided at the national, provincial and facility levels? 


\section{HELPFUL RESOURCES}

\section{STEP I: COLLECTING INFORMATION NEEDEDTO INFORM PROGRAM}

Centers for Disease Control (CDC). 1997. Principles of Community Engagement. Atlanta: CDC. http://www.cdc. gov/phppo/pce/part1.htm

Christofides, $\mathrm{N}$ et al. 2006, "How to conduct a situational analysis of health services for survivors of sexual assault." Johannesburg: Sexual Violence Research Initiative, Medical Research Council. http://www.svri.org//analysis.htm

Du Mont, J. \& White, D. 2007. The uses and impacts of medico-legal evidence in sexual assault cases: a global review. Sexual Violence Research Initiative, World Health Organization. http://www.svri.org//medico.pdf

Ellsberg M and Heise L. 2005. Researching Violence Against Women: A Practical Guide for Researchers and Activists. Washington DC, United States: World Health

Organization, PATH. http://www.path.org/files/GBV_rvaw_complete.pdf

Garcia-Moreno C. et al. 2005. WHO Multi-country Study on Women's Health and Domestic Violence. Geneva: World Health Organization. http://www.who.int/gender/violence/who_multicountry_study/en/

Raising Voices, Uganda. http://www.raisingvoices.org/

\section{STEP 2: ESTABLISHING THE REGULATORY AND PHYSICAL FRAMEWORK FOR SERVICES}

Ajema, C et al. 2009. "Standards required in maintaining the chain of evidence in the context of post-rape care services: Findings of a study conducted in Kenya." Nairobi: Liverpool VCT Care \& Treatment, the Kenyan Ministry of Health Division of Reproductive Health and the Population Council. http://www.popcouncil.org/pdfs/2009RH_ KenyaPostRapeCareServices.pdf

Center for the Study of Violence and Reconciliation and Aids Law Project. Preventing HIV after Rape: Steps you can take to protect your health. www.doh.gov.za/aids/docs/after-rape.htm

Emergency Contraception: Health Care Providers' Quick Reference Guide. Nairobi: the Kenyan Ministry of Health and the Population Council. http://www.popcouncil.org/pdfs/RH_ECQuickRefGuide.pdf

Ethiopia: National Guidelines for the Management of Survivors of Sexual Assault in Ethiopia, Ethiopian Federal Ministry of Health; Addis Ababa, 2009.

Greater Nelspruit Intervention Programme. 2008. GRIP: A guide for survivors of rape and sexual assault. http:// www.grip.org.za

Kenya, Ministry of Health, Division of Reproductive Health. 2009 (2nd ed.). National Guidelines on Medical Management of Sexual Violence in Kenya. http://www.svri.org/nationalguidelines.pdf

World Health Organization, United Nations Population Fund and United Nations High Commissioner for Refugees. 2004 (revised edition). Clinical management of rape survivors: Developing protocols for use with refugees and internally displaced persons. Geneva: World Health Organization. http://www.who.int/reproductivehealth/ publications/emergencies/924159263X/en/ 
World Health Organization. 2003. Guidelines for medico-legal care for victims of sexual violence. Geneva: World Health Organization. Geneva: World Health Organization. http://www.who.int/violence_injury_prevention/ publications/violence/med_leg_guidelines/en/index.html

\section{STEP 3:TRAINING HEALTH CARE PROVIDERS}

International Law Enforcement Academy (ILEA), Gabarone: "Policing Sexual and Gender-Based Violence," Course Materials, 2010.

Institute for Child Witness Research and Training, "Women and Children as Witnesses in cases of Gender Based Violence," Training course. www.childwitness.net

International Rescue Committee and University of California Los Angeles. 2008. Clinical Care for Sexual Assault Survivors: A multi-media training tool. http://clinicalcare.rhrc.org.

International Rescue Committee and FHI. 2000. Communication Skills in Working with Survivors of Genderbased Violence: a five day training manual. http://www.rhrc.org/resources/gbv/comm_manual/comm_manual.toc. html

Jina et al. 2008. Caring for Survivors of Sexual Assault and Rape: A Training Programme for Health Care Providers in South Africa. Pretoria: South African Department of Health. For information contact the Medical Research Council at (+27) (0) 123398527 or svri@mrc.ac.za

LVCT trauma counseling protocol. Available on www.liverpoolvct.org.

Ministry of Health, Kenya. 2007. Trainer's Manual on clinical care for survivors of sexual violence. Nairobi: Ministry of Health. Available on www.liverpoolvct.org.

Ministry of Health , Kenya. Trainer's Manual for Rape Trauma Counselors in Kenya. 2006. Nairobi: Ministry of Health. http://www.svconference2008.org/publications/Rape\%20trauma\%20counsellors\%20training\%20manual. pdf

World Health Organization, United Nations High Commissioner for Refugees and United Nations Fund for Population Activities. Clinical Management of Rape E-learning Programme. (Available in English and French.) http://iawg.net/cmor/data_en/CMoR_en.html

\section{STEP 4: INCREASING PEP ADHERENCE AND FOLLOW-UP CARE}

World Health Organization. 2003. Guidelines for medico-legal care for victims of sexual violence. Geneva: World Health Organization. Geneva: World Health Organization. http://www.who.int/violence_injury_prevention/ publications/violence/med_leg_guidelines/en/index.html

World Health Organization and International Labour Organization. 2007. Post-Exposure Prophylaxis to Prevent HIV Infection: Joint WHO/ILO Guidelines on Post-Exposure Prophylaxis to Prevent HIV Infection. Geneva: World Health Organization. http://www.who.int/hiv/pub/guidelines/PEP/en/

\section{STEP 5: DEVELOPING REFERRAL LINKAGESTO OTHER SERVICE PROVIDERS}

Bott S. et al. 2004. Improving the Health Sector Response to Gender-based Violence: A Resource Manual for Health Care Professionals in Developing Countries. London: International Planned Parenthood Federation. http:// www.hrhresourcecenter.org/node/1385 


\section{STEP 6: STRENGTHENING LINKAGES BETWEEN THE HEALTH FACILITY AND COMMUNITIES TO INCREASE TIMELY UTILIZATION OF SERVICES}

Centers for Disease Control (CDC). 1997. Principles of Community Engagement. Atlanta: CDC. http://www.cdc. gov/phppo/pce/part1.htm

IGWG of USAID. 2008, 2nd ed. Addressing Gender-based Violence through USAID's Health Programs: A Guide for Health Sector Program Officers. Washington, D.C.

Lisa VeneKlasen with Valerie Miller. 2007. A New Weave of Power, People \& Politics: The Action Guide for Advocacy and Citizen Participation. Washington: Just Associates. http://www.justassociates.org/ActionGuide.htm Raising Voices (Ugandan NGO with expertise on community-based prevention). http://www.raisingvoices.org/

World Health Organization, 2004. Preventing Violence: A Guide to Implementing the Recommendations of the World Report on Violence and Health. Geneva: WHO. http://www.who.int/violence_injury_prevention/media/ news/08_09_2004/en/index.html

World Health Organization/ London School of Hygiene and Tropical Medicine. 2010. Preventing intimate partner and sexual violence against women: taking action and generating evidence. Geneva, World Health Organization.

\section{STEP 7: MONITORING AND EVALUATING THE PROGRAM}

Bloom S. 2008. Violence Against Women and Girls: A Compendium of Monitoring and Evaluation Indicators. USAID/East Africa, IGWG and Measure Evaluation. http://www.cpc.unc.edu/measure/publications/pdf/ms-08-30. pdf

Clarumunt, M. "Helping ourselves to help others: Self-care wuide for those who work in the field of family violence” PAHO/WHO (1999). http://www.paho.org/spanish/hdp/hdw/gph7english.pdf

Ellsberg M and Heise L. 2005. Researching Violence against Women: A Practical Guide for Researchers and Activists. Washington DC: World Health Organization, PATH. http://www.path.org/files/GBV_rvaw_complete.pdf

PEPFAR Next Generation Indicators Reference Guide. August 2009. http://www.pepfar.gov/documents/ organization/81097.pdf

Reproductive Health Response in Crises Consortium. 2003. Gender-Based Violence Tools Manual: For Assessment and Program Design, Monitoring and Evaluation in Conflict-Affected Settings. New York: RHRC Consortium. http://www.rhrc.org/resources/gbv/gbv_tools/manual_toc.html

\section{CONCLUSION: PROMOTING COUNTRY OWNERSHIP, SUSTAINABILITY} AND SCALE-UP

Kilonzo N. et al. 2008. Delivery of post-rape care services: Kenya's experience in developing integrated services. http://www.who.int/bulletin/volumes/87/7/08-052340.pdf.

Expandnet/ World Health Organization. 2009. Nine-step Guide for Developing a Scale-up Strategy. http://www. expandnet.net/tools.htm 


\section{REFERENCES}

${ }^{1}$ Elson, L. and J. Keesbury. 2010. "PEPFAR special initiative on sexual and gender-based violence: Baseline report." Lusaka: Population Council.

${ }^{2}$ Christofides, C. et al. 2003. "The state of sexual assault services: findings from a situational analysis of services in South Africa." Pretoria: The South African Gender-based Violence and Health Initiative, Medical Research Council.

${ }^{3}$ Keesbury, J. and I. Askew. 2010. "Comprehensive responses to gender based violence in low-resource settings: Lessons learned from implementation.” Lusaka: Population Council.

${ }^{4}$ USAID South Africa Program in Support of PEPFAR. 2007. "Thuthuzela Care Centers: Final report on the compliance assessment of the Thuthuzela Care Centers with National Department of Health Guidelines for managing HIV in the context of sexual assault." http://sa.usaid.gov/southafrica/sites/south_africa/files/FINAL\%20 REPORT\%20ON\%20GAP\%20ANALYSIS-0.pdf.

${ }^{5}$ Keesbury, J. and L. Elson. 2010. "PEPFAR special initiative on sexual and gender-based violence: Final evaluation." Lusaka: Population Council

${ }^{6} \mathrm{Kim}$, J. et al. 2009. "The Refentse Model for post-rape care: Strengthening sexual assault care and HIV postexposure prophylaxis in a district hospital in Rural South Africa." Washington, DC: Population Council.

${ }^{7}$ Vetten, L. et al. 2009. "Policy brief \#2: From paper to practice: Lessons in the implementation of health and victim empowerment policy applicable to rape survivors." South Africa: Tswaranang Legal Advocacy Centre.

${ }^{8}$ World Health Organization. 2003. "Guidelines for medico-legal care for victims of sexual violence." Geneva: World Health Organization

${ }^{9}$ South African National Department of Health. 2004. National Management Guidelines for Sexual Assault Care. Pretoria: South African National Department of Health.

${ }^{10}$ World Health Organization, United Nations Population Fund and United Nations High Commissioner for Refugees. 2004 (revised edition). Clinical Management of Rape Survivors: Developing Protocols for Use With Refugees and Internally Displaced Persons. Geneva: World Health Organization.

${ }^{11}$ World Health Organization. 2005. "Researching violence against women: a practical guide for researchers and activists.” Geneva: World Health Organization.

${ }^{12}$ Keesbury, J. et al. 2009. "The Copperbelt Model of integrated care for survivors of rape and defilement: Testing the feasibility of police provision of emergency contraceptive pills." Lusaka: Population Council.

${ }^{13}$ Christofides, C. et al. 2005. "Other patients are really in need of medical attention: the quality of health services for rape survivors in South Africa.” Bull World Health Org 83(7): 495-502.

${ }^{14}$ Vetten, L. and S. Haffejee. 2005. "Factors affecting adherence to post-exposure prophylaxis in the aftermath of sexual assault: Key findings from seven sites in Gauteng Province."

${ }^{15} \mathrm{Du}$ Mont, J. and D. White. 2007. "The uses and impacts of medico-legal evidence in sexual assault cases: a global review.” Sexual Violence Research Initiative. Geneva: World Health Organization. 
${ }^{16}$ Ajema, C. B. Bwire, and S. Nduta. 2008. "LVCT community practices post sexual violence: Implications on the uptake of services and delivery of care: Lessons learnt from Kenya." Nairobi: Liverpool VCT, Care \& Treatment, the Kenyan Ministry of Health Division of Reproductive Health and the Population Council.

${ }^{17}$ Doggett, E. and M. Betron. 2008. "Involving communities to shape and take part in effective SGBV services and responses: A learning exchange and action planning workshop, Kampala, Uganda, June 30-July 4." Washington, DC: Health Policy Initiative, Task Order 1, Futures Group International.

${ }^{18}$ Doggett, E. 2010. "Community engagement in the PEPFAR special initiative on sexual and gender-based violence.” Washington, D.C.: Futures Group, Health Policy Initiative, Task Order 1.

${ }^{19}$ Ajema, C. et al. 2009. "Standards required in maintaining the chain of evidence in the context of post-rape care services: Findings of a study conducted in Kenya." Nairobi: Liverpool VCT Care \& Treatment, the Kenyan Ministry of Health Division of Reproductive Health and the Population Council.

${ }^{20}$ USAID/Zambia. 2010. “Evaluation of gender-based violence programming.” http://www.usaid.gov/our_work/ cross-cutting_programs/wid/pubs/Zambia_GBV_Programming_Evaluation_Aug-2010.pdf

${ }^{21}$ Campbell, R. 2001. "Mental health services for rape survivors: Current issues in therapeutic practice.” Violence Against Women Online Resources. http://www.mincava.umn.edu/documents/commissioned/campbell/campbell. pdf

${ }^{22}$ Jina, R. et al. 2008. "Caring for survivors of sexual assault and rape: A training programme for health care providers in South Africa." Pretoria: South African Department of Health.

${ }^{23}$ Kenya, Ministry of Health, Division of Reproductive Health. 2009. National Guidelines on Medical Management of Sexual Violence in Kenya, 2nd ed. Nairobi: Ministry of Health.

${ }^{24}$ IGWG of USAID. 2008. "Addressing gender-based violence through USAID's health programs: A guide for health sector program officers," Second Edition. Washington D.C.: USAID.

${ }^{25}$ PEPFAR Next Generation Indicators Reference Guide. August 2009.

${ }^{26}$ Bott, S. et al. 2004. Improving the Health Sector Response to Gender-based Violence: A Resource Manual for Health Care Professionals in Developing Countries. London: International Planned Parenthood Federation. http://www. hrhresourcecenter.org/node/1385

${ }^{27}$ Kilonzo, N. et al. 2008. "Delivery of post-rape care services: Kenya's experience in developing integrated services.” http://www.who.int/bulletin/volumes/87/7/08-052340.pdf. 


\section{APPENDIX I}

\section{SAMPLE CONSENT FORM FOR \\ MEDICO-LEGAL EXAMINATION*}

\section{SAMPLE CONSENT FORM}

\section{Name of facility}

Note to the health worker: After providing the relevant information to the patient as explained on page 40 (notes on completing the consent form), read the entire form to the patient (or his/her parent/guardian), explaining that s/he can choose to refuse any (or none) of the items listed. Obtain a signature, or a thumb print with signature of a witness.

I, (print name of survivor)

authorize the above-named health facility to perform the following (tick the appropriate boxes):

\section{Conduct a medical examination}

\section{Conduct a pelvic examination}

Collect evidence, such as body fluid samples, collection of clothing, hair combings, scrapings or cuttings of fingernails, blood sample, and photographs

Provide evidence and medical information to the police and/or courts concerning my case; this information will be limited to the results of this examination and any relevant follow-up care provided.

I understand that I can refuse any aspect of the examination I don't wish to undergo.

Signature:

Date:

Witness:

Source:World Health Organization (WHO), United Nations Population Fund and United Nations High Commissioner for Refugees. 2004 (revised edition). "Clinical management of rape survivors: Developing protocols for use with refugees and internally displaced persons.” Geneva: WHO. 


\section{APPENDIX 2}

SAMPLE FACILITY INVENTORY TOOL*

Instructions to the data collector: Please request that the in-charge of each facility assist you in completing this form. The objectives of this process are to:

- Assess the facility's physical capacity to provide comprehensive SV services; and

- Assist the in-charge in identifying areas for improvement in the facility infrastructure.

Remember that the objective of the inventory is to identify equipment and facilities that currently exist and are in working order-and not to evaluate the performance of the staff or clinic.

You are required to directly observe the conditions indicated on this form. In all cases you should verify that the items exist by actually observing them yourself-if you are not able to observe them, then code accordingly. For each item, circle the code most suitable response or describe as appropriate. Some questions will require additional information to be written in the indicated blank.

\begin{tabular}{|c|c|c|c|}
\hline \multicolumn{3}{|c|}{ Section I: Facility identification } & \multirow{2}{*}{$\begin{array}{l}\text { Write number } \\
\text { here }\end{array}$} \\
\hline 1.1 & Name of data collector & Write name & \\
\hline 1.2 & $\begin{array}{l}\text { Was in-charge or } \mathrm{HC} \text { manager present for } \\
\text { entire observation? }\end{array}$ & $\begin{array}{l}\text { No.................................... } \\
\text { Yes.............................. }\end{array}$ & \\
\hline 1.3 & Date of observation & $\frac{l}{\mathrm{DD}} \frac{1}{\mathrm{MM} Y Y Y}$ & \\
\hline 1.4 & Start time & $\frac{l}{\mathrm{HH}} \frac{}{M M}$ & \\
\hline 1.5 & End time & $\frac{\mathrm{l}}{\mathrm{HH}} \frac{}{\mathrm{MM}}$ & \\
\hline 1.6 & Type of observation & $\begin{array}{l}\text { Baseline } \ldots \ldots \ldots \ldots \ldots \ldots \ldots \ldots \ldots \ldots \ldots \ldots \ldots \ldots \ldots \ldots \ldots \ldots \ldots \ldots \ldots \ldots \ldots \ldots \ldots \\
\text { Endline } \ldots \ldots \ldots \ldots \ldots \ldots \ldots\end{array}$ & \\
\hline 1.7 & Name of facility & Write name & \\
\hline 1.8 & PEPFAR partner name & Write name & \\
\hline
\end{tabular}




\section{Section 2: Service availability}

This section should be completed with the assistance of the facility-in charge or knowledgeable staff who can provide the following information. No direct observations are required for this section.

\begin{tabular}{|c|c|c|}
\hline 2.1 & $\begin{array}{l}\text { In what departments/locations are SV services PRIMARILY } \\
\text { provided in this facility? }\end{array}$ & $\begin{array}{l}\text { List up to three. } \\
\text { I. } \\
2 . \\
3 .\end{array}$ \\
\hline 2.2 & $\begin{array}{l}\text { Is there one specific area or room dedicated for SV } \\
\text { services? }\end{array}$ & $\begin{array}{l}\text { No.................... } \\
\text { Yes.................. }\end{array}$ \\
\hline
\end{tabular}

\begin{tabular}{|c|c|}
\hline $\begin{array}{l}\text { a. In what department/location* is this area or room } \\
\text { located? }\end{array}$ & $\begin{array}{l}\text { Write name } \\
\text { *this should be one of the } \\
\text { departments/locations listed in } \\
\text { 3.I }\end{array}$ \\
\hline b. What services are offered in this area or room? & $\begin{array}{l}\text { Medical care.................... } \\
\text { HIV and PEP services.........2 } \\
\text { Emergency contraception...3 } \\
\text { Forensic examination.........4 } \\
\text { Counseling.......................5 } \\
\text { Police services..................6 }\end{array}$ \\
\hline $\begin{array}{l}\text { c. On WEEKDAYS, during what hours are SV services } \\
\text { available in this area or room? }\end{array}$ & $\begin{array}{l}\text { Opening time } \frac{1}{\mathrm{HH} / \mathrm{MM}} \\
\text { Closing time }\end{array}$ \\
\hline $\begin{array}{l}\text { d. On WEEKENDS/PUBLIC HOLIDAYS during what hours } \\
\text { are SV services available in this area or room? }\end{array}$ & $\begin{array}{l}\text { Opening time } \frac{\mathrm{I}}{\mathrm{HH} / \mathrm{MM}} \\
\text { Closing time }\end{array}$ \\
\hline $\begin{array}{l}\text { e. Is this area or room available at all times SV services are } \\
\text { offered? }\end{array}$ & $\begin{array}{l}\text { No..................... } \\
\text { Yes.................. }\end{array}$ \\
\hline $\begin{array}{l}\text { f. Is there a doctor available in the facility at all times that } \\
\text { SV services are available in this area or room? }\end{array}$ & $\begin{array}{l}\text { No.................... } \\
\text { Yes................... }\end{array}$ \\
\hline $\begin{array}{l}\text { g. Is there a nurse available in the facility at all times that } \\
\text { SV services are offered in this area or room? }\end{array}$ & $\begin{array}{l}\text { No.................... } \\
\text { Yes................... }\end{array}$ \\
\hline $\begin{array}{l}\text { h. Is there a counselor available in the facility at all times } \\
\text { that SV services are offered in this area or room? }\end{array}$ & $\begin{array}{l}\text { No...................... } \\
\text { Yes.................... }\end{array}$ \\
\hline
\end{tabular}




\begin{tabular}{|c|c|c|}
\hline & $\begin{array}{l}\text { i. Is there a police officer available in the facility at all } \\
\text { times that SV services are offered in this area or room? }\end{array}$ & $\begin{array}{l}\text { No....................... } \\
\text { Yes.................... }\end{array}$ \\
\hline \multirow[t]{3}{*}{2.3} & $\begin{array}{l}\text { Are SV services offered elsewhere in the facility if the } \\
\text { dedicated SV area or room is not available? }\end{array}$ & $\begin{array}{l}\text { No....................... } \\
\text { Yes..................... }\end{array}$ \\
\hline & $\begin{array}{l}\text { Where are SV services offered when the dedicated SV area } \\
\text { or room is not available? }\end{array}$ & $\begin{array}{l}\text { Write name } \\
\text { *this should be one of the } \\
\text { departments/locations listed in } \\
3.1\end{array}$ \\
\hline & $\begin{array}{l}\text { What services are offered when the dedicated SV area or } \\
\text { room is not available? }\end{array}$ & $\begin{array}{l}\text { Medical care................... I } \\
\text { HIV and PEP services.........2 } \\
\text { Emergency contraception..3 } \\
\text { Forensic examination.........4 } \\
\text { Counseling.......................5 } \\
\text { Police services..................6 }\end{array}$ \\
\hline \multirow[t]{7}{*}{2.4} & $\begin{array}{l}\text { If there is no dedicated room available for SV services, in } \\
\text { what department/location are SV medical examinations } \\
\text { most frequently conducted? }\end{array}$ & $\begin{array}{l}\text { Write name } \\
\text { *this should be one of the } \\
\text { departments/locations listed in } \\
3.1\end{array}$ \\
\hline & $\begin{array}{l}\text { On WEEKDAYS, during what hours can SV medical } \\
\text { examinations be performed in this department/location? }\end{array}$ & 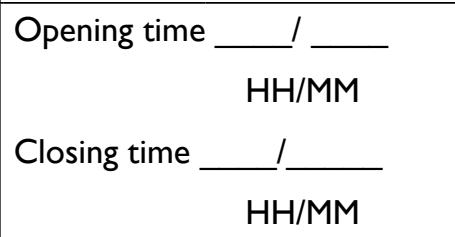 \\
\hline & $\begin{array}{l}\text { On WEEKENDS/PUBLIC HOLIDAYS during what hours can } \\
\text { SV medical examinations be performed in this department/ } \\
\text { location? }\end{array}$ & 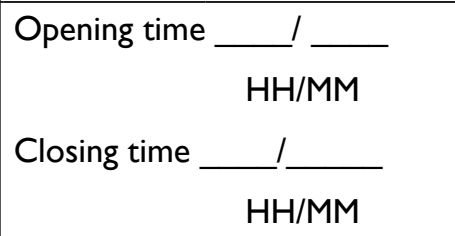 \\
\hline & $\begin{array}{l}\text { Is there a doctor available in the facility at all times when } \\
\text { medical examinations can be performed? }\end{array}$ & $\begin{array}{l}\text { No...................... } \\
\text { Yes.................... }\end{array}$ \\
\hline & $\begin{array}{l}\text { Is there a nurse available in the facility at all times when } \\
\text { medical examinations can be performed? }\end{array}$ & $\begin{array}{l}\text { No...................... } \\
\text { Yes.................... }\end{array}$ \\
\hline & $\begin{array}{l}\text { Is there counselor available in the facility at all times when } \\
\text { medical examinations can be performed? }\end{array}$ & $\begin{array}{l}\text { No..................... } \\
\text { Yes.................... }\end{array}$ \\
\hline & $\begin{array}{l}\text { Is there a police officer available in the facility at all times } \\
\text { when medical examinations can be performed? }\end{array}$ & $\begin{array}{l}\text { No..................... } \\
\text { Yes.................... }\end{array}$ \\
\hline
\end{tabular}




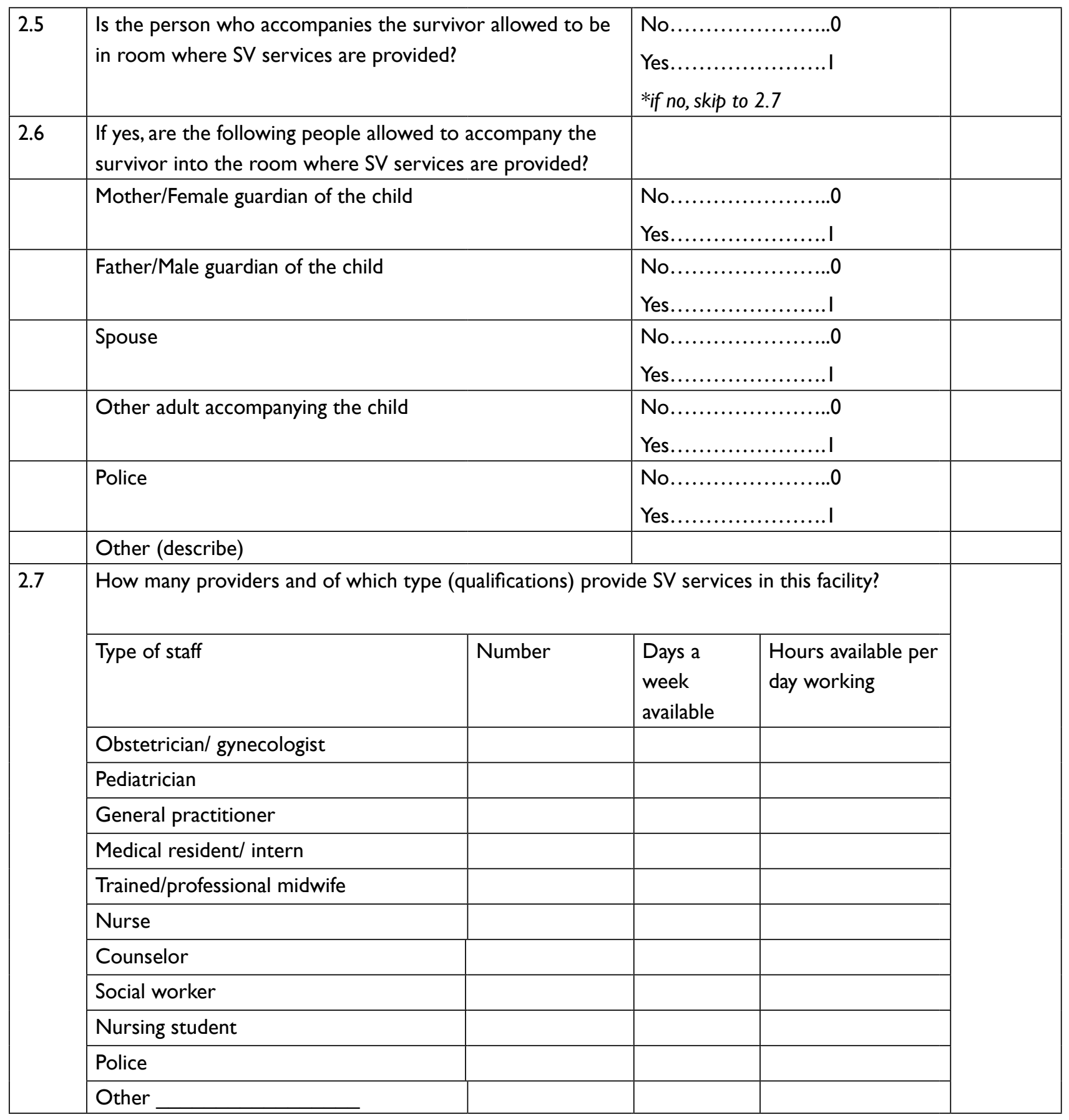




\begin{tabular}{|c|c|c|c|}
\hline \multicolumn{4}{|c|}{$\begin{array}{l}\text { Section 3: General infrastructure } \\
\text { For this section, you will need to physically see these areas to properly answer the questions. }\end{array}$} \\
\hline 3.1 & $\begin{array}{l}\text { Is there a separate area where SV clients wait to receive } \\
\text { services? }\end{array}$ & $\begin{array}{l}\text { No...................... } \\
\text { Yes..................... } \\
\text { Did not observe.......9 }\end{array}$ & \\
\hline \multirow[t]{3}{*}{3.2} & In the area where SV clients receive medical services, can: & & \\
\hline & Conversations be heard from the outside? & $\begin{array}{l}\text { No...................... } \\
\text { Yes..................... } \\
\text { Did not observe.......9 }\end{array}$ & \\
\hline & Clients be seen from the outside? & $\begin{array}{l}\text { No...................... } \\
\text { Yes.....................। } \\
\text { Did not observe.......9 }\end{array}$ & \\
\hline \multirow[t]{5}{*}{3.3} & $\begin{array}{l}\text { Is there one specific area or room dedicated for SV } \\
\text { counseling? }\end{array}$ & $\begin{array}{l}\text { No.................... } \\
\text { Yes..................... } \\
\text { Did not observe.......9 } \\
\text { *If no or did not observe, skip to } \\
\text { section } 4\end{array}$ & \\
\hline & $\begin{array}{l}\text { Is this area or room different from the medical consultation } \\
\text { area? }\end{array}$ & $\begin{array}{l}\text { No..................... } \\
\text { Yes.................... } \\
\text { Did not observe.......9 }\end{array}$ & \\
\hline & $\begin{array}{l}\text { Is this area or room available at all times SV services are } \\
\text { offered? }\end{array}$ & $\begin{array}{l}\text { No..................... } \\
\text { Yes.................... } \\
\text { Did not observe.......9 }\end{array}$ & \\
\hline & Can conversations be heard from the outside? & $\begin{array}{l}\text { No..................... } \\
\text { Yes.................... } \\
\text { Did not observe.......9 }\end{array}$ & \\
\hline & Can clients be seen from the outside? & $\begin{array}{l}\text { No..................... } \\
\text { Yes....................। } \\
\text { Did not observe........9 }\end{array}$ & \\
\hline
\end{tabular}




\section{Section 4: Essential equipment and supplies}

Now go to the area where SV medical examinations routinely take place. This should be the location identified in question 2.2 a (the dedicated area or room for SV services.) If there is not a dedicated room, go to the location identified in question 2.4 (the department department/location where SV medical examinations are most frequently conducted).

\begin{tabular}{|c|c|c|}
\hline 4.1 & Name of location where this inventory is taking place & $\begin{array}{l}\text { Write name } \\
\text { *this should correspond with the location } \\
\text { listed in questions } 2.2 a \text { OR } 2.4 \text { above }\end{array}$ \\
\hline 4.2 & Is there a working angle lamp? & $\begin{array}{l}\text { No..................... } \\
\text { Yes.................... } \\
\text { Did not observe.......9 }\end{array}$ \\
\hline 4.3 & Is there an examination couch? & $\begin{array}{l}\text { No..................... } \\
\text { Yes.................... } \\
\text { Did not observe.......9 }\end{array}$ \\
\hline 4.4 & Is there a speculum? & $\begin{array}{l}\text { No....................... } \\
\text { Yes...................... } \\
\text { Did not observe........9 }\end{array}$ \\
\hline 4.5 & Are there examination gloves? & $\begin{array}{l}\text { No...................... } \\
\text { Yes...................... } \\
\text { Did not observe........9 }\end{array}$ \\
\hline 4.6 & Is there a sharps container? & $\begin{array}{l}\text { No....................... } \\
\text { Yes...................... } \\
\text { Did not observe........9 }\end{array}$ \\
\hline 4.7 & $\begin{array}{l}\text { Is there a lockable cupboard for the storage of forensic/ } \\
\text { medico-legal evidence? }\end{array}$ & $\begin{array}{l}\text { No..................... } \\
\text { Yes.................... } \\
\text { Did not observe.......9 } \\
\end{array}$ \\
\hline 4.8 & Is there a lockable medical supply cabinet? & $\begin{array}{l}\text { No..................... } \\
\text { Yes..................... } \\
\text { Did not observe.......9 } \\
\end{array}$ \\
\hline 4.9 & Are there sanitary towels? & $\begin{array}{l}\text { No..................... } \\
\text { Yes..................... } \\
\text { Did not observe.......9 } \\
\end{array}$ \\
\hline 4.10 & Is there emergency clothing? & $\begin{array}{l}\text { No...................... } \\
\text { Yes..................... } \\
\text { Did not observe........9 }\end{array}$ \\
\hline
\end{tabular}




\begin{tabular}{|c|c|c|}
\hline 4.11 & Is there a consent form for the examination? & $\begin{array}{l}\text { No..................... } \\
\text { Yes................... } \\
\text { Did not observe.......9 }\end{array}$ \\
\hline 4.12 & Are there swabs? & $\begin{array}{l}\text { No } \ldots \ldots \ldots \ldots \ldots \ldots \ldots \\
\text { Yes.....................। } \\
\text { Did not observe........9 }\end{array}$ \\
\hline 4.13 & Are there blood tubes? & $\begin{array}{l}\text { No...................... } \\
\text { Yes...................... } \\
\text { Did not observe.......9 }\end{array}$ \\
\hline 4.14 & $\begin{array}{l}\text { Are there special aids for examining children (dolls, paper } \\
\text { and pens for drawing pictures?) }\end{array}$ & $\begin{array}{l}\text { No..................... } \\
\text { Yes.................... } \\
\text { Did not observe........9 }\end{array}$ \\
\hline 4.15 & Is there a pregnancy test kit? & 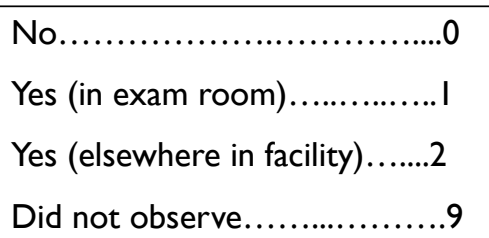 \\
\hline 4.14 & Are there emergency contraceptive pills? & $\begin{array}{l}\text { No } \\
\text { Yes (in exam room) ............... } \\
\text { Yes (elsewhere in facility)........2 } \\
\text { Did not observe...................9. }\end{array}$ \\
\hline & $\begin{array}{l}\text { If yes, what brand of emergency contraceptive pills are } \\
\text { available? }\end{array}$ & (write name) \\
\hline 4.15 & Are there STI prophylaxis/treatment? & 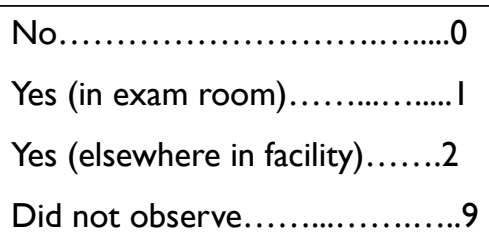 \\
\hline 4.16 & Is there analgesia? & 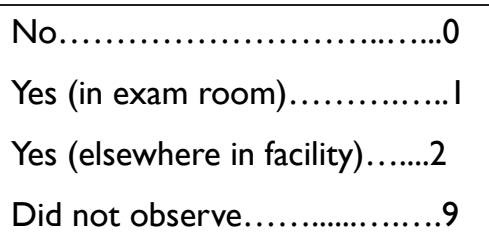 \\
\hline 4.17 & Are there tranquilizers? & 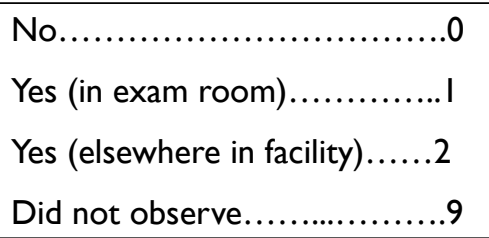 \\
\hline 4.18 & Are there anti-emetics? & 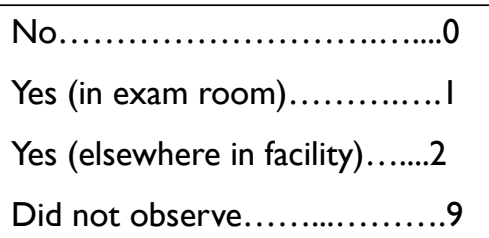 \\
\hline
\end{tabular}




\section{Section 5: Protocols and information for patients}

Remain in the area where SV medical examinations routinely take place. This should be the location identified in question 2.2 a (the dedicated area or room for SV services.) If there is not a dedicated room, go to the location identified in question 2.4 (the department department/location where SV medical examinations are most frequently conducted).

\begin{tabular}{|c|c|c|}
\hline 5.1 & Name of location where this inventory is taking place & $\begin{array}{l}\text { Write name } \\
\text { *this should correspond with the location } \\
\text { listed in } 4.1 \text { above }\end{array}$ \\
\hline 5.2 & $\begin{array}{l}\text { Do facility registers clearly indicate if a client presents for } \\
\text { SV services? }\end{array}$ & $\begin{array}{l}\text { No..................... } \\
\text { Yes..................... } \\
\text { Did not observe........ }\end{array}$ \\
\hline 5.3 & $\begin{array}{l}\text { Do facility records indicate if a client receives follow-up } \\
\text { care? }\end{array}$ & $\begin{array}{l}\text { No..................... } \\
\text { Yes..................... } \\
\text { Did not observe........ }\end{array}$ \\
\hline 5.4 & $\begin{array}{l}\text { Do referral guidelines explicitly address procedures for } \\
\text { removing a child from an unsafe domestic environment? }\end{array}$ & $\begin{array}{l}\text { No............................... } \\
\text { Yes........................... }\end{array}$ \\
\hline 5.5 & $\begin{array}{l}\text { Does the facility have a copy of the national guidelines for } \\
\text { providing SV care located in or nearby the room where } \\
\text { medical examinations routinely take place? }\end{array}$ & $\begin{array}{l}\text { No......................... } \\
\text { Yes....................... } \\
\text { National guidelines do not exist.....2 } \\
\text { National guidelines exist but did not } \\
\text { observe.......9 }\end{array}$ \\
\hline 5.6 & $\begin{array}{l}\text { Does the facility have a copy of the facility-specific } \\
\text { guidelines for providing SV care located in or nearby the } \\
\text { room where medical examinations routinely take place? }\end{array}$ & $\begin{array}{l}\text { No......................... } \\
\text { Yes....................... } \\
\text { Facility guidelines do not exist.....2 } \\
\text { Facility guidelines exist but did not } \\
\text { observe....... }\end{array}$ \\
\hline 5.7 & $\begin{array}{l}\text { Are there special guidelines for examining and treating } \\
\text { infants and children? }\end{array}$ & $\begin{array}{l}\text { No................................. } \\
\text { Yes, included in national guidelines.... } \\
\text { Yes, included in facility guidelines.....2 } \\
\text { Yes, exist on their own.... } 3 \\
\text { Exist but did not observe...9 }\end{array}$ \\
\hline 5.8 & $\begin{array}{l}\text { Are there leaflets or handouts on medication provided for } \\
\text { SV survivors? }\end{array}$ & $\begin{array}{l}\text { No } \ldots \ldots \ldots \ldots \ldots \ldots \ldots \ldots \ldots \ldots \\
\text { Yes................................ } \\
\text { Did not observe............... }\end{array}$ \\
\hline 5.9 & $\begin{array}{l}\text { Are there leaflets and handouts for rape survivors on } \\
\text { support services, such as NGOs? }\end{array}$ & 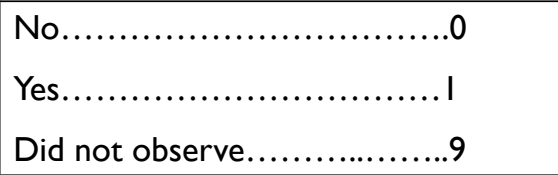 \\
\hline
\end{tabular}




\begin{tabular}{|c|c|c|c|}
\hline 5.10 & $\begin{array}{l}\text { Does the facility have written guidelines for referral of } \\
\text { survivors to other services located in or nearby the room } \\
\text { where medical examinations routinely take place? }\end{array}$ & 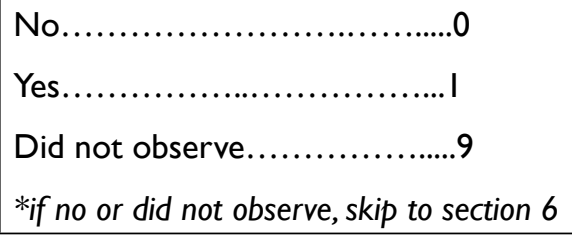 & \\
\hline \multirow[t]{7}{*}{5.11} & \multicolumn{2}{|l|}{ What services are included in the referral guidelines? } & \\
\hline & Police & $\begin{array}{l}\text { No............................... } \\
\text { Yes............................. }\end{array}$ & \\
\hline & Safe house & $\begin{array}{l}\text { No } \ldots \ldots \ldots \ldots \ldots \ldots \ldots \ldots \ldots \ldots \ldots \ldots \\
\text { Yes..................................... }\end{array}$ & \\
\hline & Legal services & 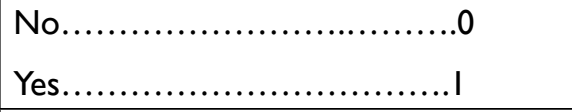 & \\
\hline & Counseling/ psychosocial support & $\begin{array}{l}\text { No.................................. } \\
\text { Yes............................ }\end{array}$ & \\
\hline & HIVIAIDS care and treatment & $\begin{array}{l}\text { No................................ } \\
\text { Yes............................. }\end{array}$ & \\
\hline & Other & $\begin{array}{l}\text { No............................... } \\
\text { Yes........................... }\end{array}$ & \\
\hline 5.12 & $\begin{array}{l}\text { Do the referral guidelines include phone numbers and } \\
\text { contact people at each referral point? }\end{array}$ & $\begin{array}{l}\text { No } \ldots \ldots \ldots \ldots \ldots \ldots \ldots \ldots \ldots \ldots \ldots \ldots \\
\text { Yes..................................... }\end{array}$ & \\
\hline \multicolumn{4}{|c|}{$\begin{array}{l}\text { Section 6: HIV services } \\
\text { Now go to the area where HIV services are routinely offered to SV survivors. This may be the same location identified in questions 2.2, } \\
2.3 \text { or } 2.4 \text { above. It may also be a different location in the facility. }\end{array}$} \\
\hline 6.1 & $\begin{array}{l}\text { Where are HIV services provided to survivors of sexual } \\
\text { violence? }\end{array}$ & Write name & \\
\hline 6.2 & Name of location where this inventory is taking place & $\begin{array}{l}\text { Write name } \\
\text { *should be same location as in } 6.1\end{array}$ & \\
\hline \multirow[t]{3}{*}{6.3} & $\begin{array}{l}\text { Is this the same location where the medical exam for SV is } \\
\text { preformed? }\end{array}$ & $\begin{array}{l}\text { No................................. } \\
\text { Yes............................ }\end{array}$ & \\
\hline & $\begin{array}{l}\text { On WEEKDAYS, during what hours are HIV services } \\
\text { available in this area or room? }\end{array}$ & $\begin{array}{l}\text { Opening time } \frac{1}{\mathrm{HH} / \mathrm{MM}} \\
\text { Closing time } \frac{\mathrm{H}}{\mathrm{H} / \mathrm{MM}}\end{array}$ & \\
\hline & $\begin{array}{l}\text { On WEEKENDS/ PUBLIC HOLIDAYS during what hours } \\
\text { are HIV services available in this area or room? }\end{array}$ & $\begin{array}{l}\text { Opening time } \frac{\mathrm{l}}{\mathrm{HH} / \mathrm{MM}} \\
\text { Closing time } \frac{\mathrm{I}}{\mathrm{H} / \mathrm{MM}}\end{array}$ & \\
\hline
\end{tabular}




\begin{tabular}{|c|c|c|}
\hline & $\begin{array}{l}\text { Where are HIV services provided when this location is } \\
\text { closed? }\end{array}$ & $\begin{array}{l}\text { In another location within this } \\
\text { facility................................. } \\
\text { In another facility................2 } \\
\text { Services not available..........3 }\end{array}$ \\
\hline 6.4 & Is there a HIV rapid test kit? & 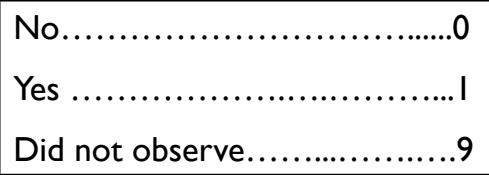 \\
\hline 6.5 & $\begin{array}{l}\text { Are there post-exposure prophylaxis (PEP) drugs to } \\
\text { prevent HIV? }\end{array}$ & 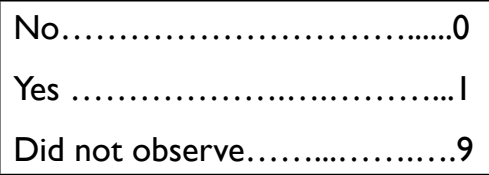 \\
\hline 6.6 & $\begin{array}{l}\text { How many days of PEP drugs are provided during initial } \\
\text { visit? }\end{array}$ & $\begin{array}{l}3 \text { days of pills............... } \\
28 \text { days of pills...............2 } \\
\text { Other............................ }\end{array}$ \\
\hline 6.7 & $\begin{array}{l}\text { Are children referred to another location for pediatric } \\
\text { PEP? }\end{array}$ & $\begin{array}{l}\text { No } \ldots \ldots \ldots \ldots \ldots \ldots \ldots \ldots \ldots \ldots \ldots \ldots \\
\text { Yes.............................. } \\
\text { Did not know................ }\end{array}$ \\
\hline 6.8 & $\begin{array}{l}\text { When are PEP clients advised to return to the facility for a } \\
\text { follow-up HIV test? }\end{array}$ & _ weeks \\
\hline
\end{tabular}

Thank you very for your time and contribution to this survey!

Adapted from Christofides, N. et al. 2006. "How to conduct a situational analysis of health services for survivors of sexual assault.” Johannesburg: Sexual Violence Research Initiative, Medical Research Council. http://www.svri.org//analysis.htm 


\section{APPENDIX 3}

\section{SAMPLE GUIDE FOR FGDS WITH SERVICE PROVIDERS}

NOTE TO THE FACILITATIOR: After completing informed consent procedures, the facilitator will conduct a semi-structured discussion using the following questions as a guide. Prompting questions will be used to elicit an open-ended response, which can then be directed using probing questions as needed.

The note taker should include the following information at the beginning of each session's transcript.

\begin{tabular}{|l|l|}
\hline Country & $\begin{array}{l}\text { Rwanda } \\
\text { Uganda } \\
\text { South Africa }\end{array}$ \\
\hline FGD date & DD/ MM/YY \\
\hline Facility/facilities involved & I. \\
\hline FGD facilitator/note taker & 2. \\
\hline Location of FGD & $\begin{array}{l}\text { Doctors } \\
\text { Cadre of providers }\end{array}$ \\
\hline Courses \\
\hline Cotal number of participants & HH/MM \\
\hline Start time & \begin{tabular}{l} 
HH/MM \\
\hline End time
\end{tabular} \\
\hline $\begin{array}{l}\text { Brief description of the FGD environment and location: if people were enthusiastic to participate or seemed to be forced by } \\
\text { the manager, if there were interruptions, etc. }\end{array}$ \\
\hline
\end{tabular}


General information and warm-up questions

I. When we talk about SV, what do we mean?

Probe: Does this include rape survivors, children, men, victims of intimate partner violence?

2. Do you think SV is on the rise?

Probe:Why or why not?

3. Does your (this) facility receive many SV clients?

Probe: How many is "many"? At what times are they most likely to report? What are their ages?

Informational statement: today, when we are discussing SV we are using the World Health Organization's definition as "any sexual act, attempt to obtain a sexual act, unwanted sexual comments or advances, or acts to traffic women's sexuality, using coercion, threats of harm or physical force, but any person regardless of relationship to the survivor, in any setting, including but not limited to home and work."

In other words, this includes rape and defilement (and attempts to rape and defile) that occurs to women and men, some of whom may be sexually abused by their partner, spouse, or other family members. Under your country's laws, rape is defined as (insert here); and defilement is defined as (insert here).

\section{Capacity of providers}

4. Have providers in this project/facility ever received formal training in treating SV clients?

Probe: How many? What cadres? When was this training provided? What did it cover? Was it sufficient?

5. What are the most common injuries and medical risks associated with the SV clients you see here?

Probe: Which are the most serious? Which are the least important?

6. Describe the procedure for managing a SV client.

Probe for: HIV testing and PEP provision, pregnancy testing and emergency contraception provision, STI prophylaxis, treatment of injuries and collection of forensic evidence.

7. What problems do you face in providing that care?

8. Apart from medical care, what are the other needs of SV clients? How do you assess these needs? Do clients usually receive this other care? How do they know where to go for this care? Do you know where they can go for these services?

Probe for: protocols and practices for screening and referrals to police, legal services, shelter, psychosocial care, other services.

Provider attitudes toward clients

9. Do you think SV should be treated as a medical emergency?

Probe: Should clients get treated immediately when arriving to the health facility? Why or why not? 
10. Thinking about the incident itself, are there times when a client's behavior causes the rape?

Probe: If yes, what are these behaviors (drinking alcohol, wearing revealing clothing, going out alone at night, etc.)? If her/his behavior contributes to the rape, is s/he entitled to the same medical care as someone who might be less to blame?

Linkages with community/stakeholder groups

II.SV victims often face barriers in accessing medical services. Are you aware of any barriers members of this community face in getting medical care if they are raped or defiled? If yes, what are they? How did you learn about them?

12. Do you or staff at this facility conduct outreach activities with the community in order to let them know rape care services are available here and to help get victims to medical services?

Probe: If yes, please describe these efforts.

13. Are you aware of any other groups (such as, government offices, civil society organizations, or social support services) that do community sensitization on SV?

Probe: If yes, please name the groups and what they do. Do their messages include information on your SV services and how to access them?

14. Do you or staff at this facility ever work directly with clients or with other groups to find out how well your services are meeting SV client needs?

Probe: If yes, please describe this work. Have you used this information to improve your services? 


\section{APPENDIX 4}

\section{SAMPLE INFORMED CONSENT FORM FOR PARTICIPATION IN FGDS}

\section{Consent to be in a Study on Health Services for Gender-Based Violence Survivors}

The (insert name of organization) invite you to be in a research study. Please ask questions if there is anything you do not understand.

\section{Study purpose}

The purpose of this study is to improve health care for survivors of sexual violence in a set of pilot sites across the country. It is expected that these sites will serve as a model for improving sexual violence care across the country.

\section{Your involvement}

We request that you participate in a focus group discussion that will last approximately two hours. Each group will include about 8 or 10 people. During this discussion, we will ask questions about your experiences with providing health services to sexual violence survivors. We will not ask any questions about your own personal of family history with sexual violence.

Your participation is entirely voluntary. It does not have any impact on your job or conditions of service. You are not required to participate and you are able to leave the group at any time if you feel uncomfortable with the topics covered. Leaving the focus group will not have any impact on the status of your employment with the health center. Your supervisors will not know if you do not participate in this study.

\section{Audio recording and confidentiality}

This discussion will be tape recorded. The recording will only be used by the interviewers, and will be stored under lock and key.

All information you give will be kept confidential by the interviewers. Your name will not be connected with any of the results of this study. 


\section{Risks and benefits}

It is possible that information disclosed during this discussion may be repeated outside the group by other participants. If you find the discussion upsetting in any way, the data collectors are able to put you in contact with someone in your area who can provide counseling.

You will receive reimbursement for transportation to the location where the discussion is held, and snacks and drinks will be provided.

\section{Questions?}

If you have any questions about this study or how this information will be used, please contact:

(Insert contacts of principal investigator)

You can also contact this person if you need assistance in dealing with any sexual violence that you or a family member may experience.

If you have any questions about your rights as a participant under this study, please contact:

(insert contacts of local ethical board that approved the research)

I have been told about this study. I have been allowed to ask questions. I had all of my questions answered. I would like to be in this study. By signing the form, I agree to be in the study. 


\section{APPENDIX 5}

\section{MEDICO-LEGAL FORM DEVELOPED FOR USE IN THE SGBV INITIATIVE*}

\section{SV Client Assessment Form}

\section{Section I: Survivor Details}

\begin{tabular}{|l|l|l|}
\hline I. First name: & Last name: \\
\hline 2. Physical address: & \multicolumn{2}{l|}{} \\
\hline 3. Mobile telephone number: & \\
\hline 4. Next of Kin: & 6. Date of Birth $(\mathrm{dd} / \mathrm{mm} / \mathrm{yy})$ & 7.Age (years) \\
\hline 5. Sex: $\mathrm{M} / \mathrm{F}$ &
\end{tabular}

\section{Section 2: Examination Details}

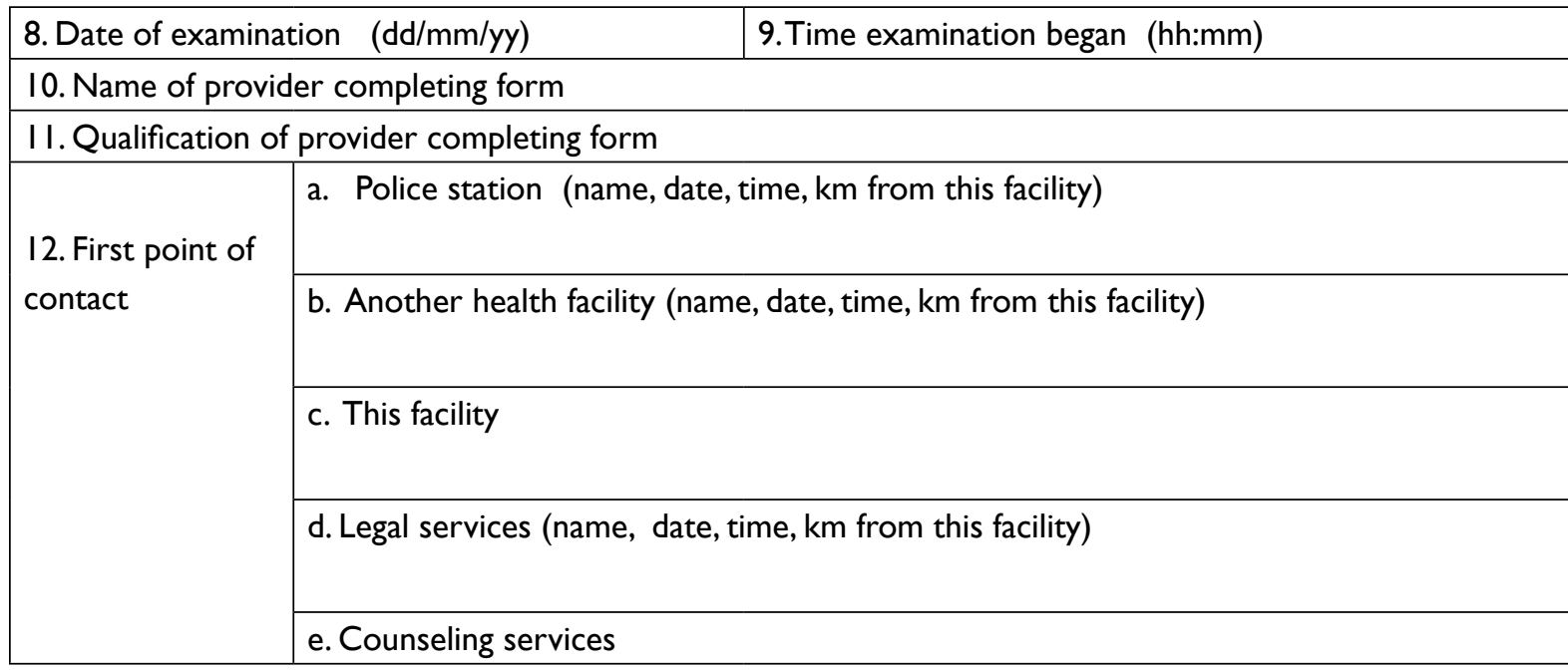




\section{Section 3: Incident History}

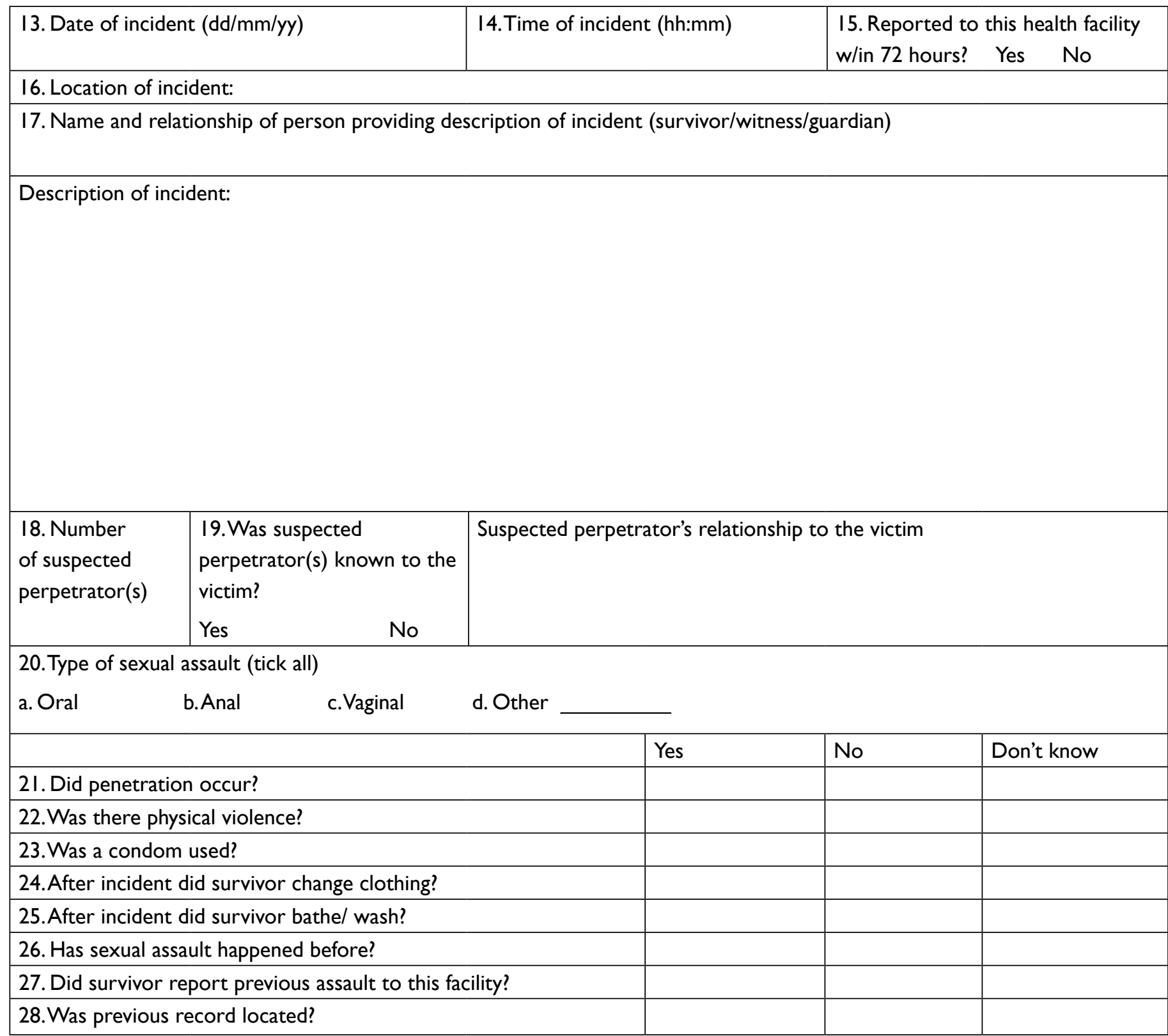




\section{Section 4: Medical History}

\begin{tabular}{|l|l|l|l|l|l|}
\hline \multicolumn{2}{|l|}{ Contraception Type: } & \multicolumn{2}{l|}{ 29. LNMP: } & 30. Date of last consensual sex: \\
\hline Weight: & Height: & BP: & $\begin{array}{r}\text { Temp } \\
\text { C }\end{array}$ & RR & Pulse \\
\hline
\end{tabular}

Relevant medical history and medications:

Any symptoms noted after incident:

(i.e., genital bleeding, genital tear, genital itching, other)

Clinical evidence of drugs or alcohol 


\section{Section 5: Medical Examination}

3I. Number and qualifications of all medical professionals present:

\begin{tabular}{|c|c|c|c|}
\hline \multicolumn{4}{|c|}{ A. Gynecological examination } \\
\hline \multirow{2}{*}{$\begin{array}{l}\text { Breast development: } \\
\text { Tanner stage I-5 }\end{array}$} & \multirow{2}{*}{\multicolumn{2}{|c|}{$\begin{array}{l}\text { Pubic hair: } \\
\text { Tanner stage I-5 }\end{array}$}} & Mons pubis: \\
\hline & & & \\
\hline Clitoris & \multicolumn{3}{|l|}{ Frenulum of clitoris } \\
\hline Urethral orifice & \multicolumn{3}{|l|}{ Para-urethral folds } \\
\hline Labia majora & \multicolumn{2}{|l|}{ Labia minora } & \\
\hline \multirow{2}{*}{$\begin{array}{l}\text { Posterior } \\
\text { fourchette: }\end{array}$} & scaring & & tears \\
\hline & \multicolumn{2}{|l|}{ bleeding } & increased friability \\
\hline \multicolumn{4}{|l|}{ Fossa navicularis } \\
\hline \multirow{3}{*}{$\begin{array}{l}\text { Hymen: } \\
\text { configuration }\end{array}$} & Opening diameter $(\mathrm{mm})$ & Transverse & Vertical \\
\hline & Swelling & Bumps & Clefts \\
\hline & Fresh tears (position) & Synechiae & Bruising \\
\hline \multirow[t]{2}{*}{ Vagina } & \multicolumn{2}{|l|}{ Number of fingers admitted } & Bleeding \\
\hline & \multicolumn{2}{|l|}{ Discharge } & Tears \\
\hline \multirow[t]{2}{*}{ Cervix } & \multicolumn{2}{|l|}{ Erosion } & Discharge \\
\hline & \multicolumn{2}{|l|}{ Bleeding } & Other \\
\hline
\end{tabular}




\begin{tabular}{|c|c|c|c|c|}
\hline \multicolumn{5}{|l|}{ B.Anal examination } \\
\hline \multirow[t]{3}{*}{ Skin surrounding the orifice } & Hygiene & \multicolumn{2}{|l|}{ Abrasions } & Redness/ erythema \\
\hline & Pigmentation & \multicolumn{2}{|l|}{ Scars } & Bruising/ hematoma \\
\hline & Fissures/ cracks & \multicolumn{2}{|l|}{ Swelling/ thickening } & Tags \\
\hline \multirow[t]{3}{*}{ Orifice } & Tears/ fissures & \multicolumn{2}{|l|}{ Reflex dilation } & Twitchiness/ winking \\
\hline & $\begin{array}{l}\text { Swelling/ thickening of rim } \\
\text { (tyre sign) }\end{array}$ & \multicolumn{2}{|c|}{$\begin{array}{l}\text { Shortening/ eversion of anal } \\
\text { canal }\end{array}$} & Discharge \\
\hline & Funneling & \multicolumn{2}{|l|}{ Cupping } & \\
\hline \multirow[t]{2}{*}{ Digital examination } & $\begin{array}{l}\text { Presence of hard feces in } \\
\text { rectum }\end{array}$ & \multicolumn{3}{|c|}{ Thickening of anal verge } \\
\hline & $\begin{array}{l}\text { Laxity (pressure on anal } \\
\text { orifice) }\end{array}$ & \multicolumn{3}{|l|}{ Tone (sphincter grip) } \\
\hline \multicolumn{5}{|c|}{ C. Examination of male genitalia } \\
\hline $\begin{array}{l}\text { Genital development: } \\
\text { Tanner stage I-5 }\end{array}$ & \multicolumn{2}{|l|}{$\begin{array}{l}\text { Pubic hair: } \\
\text { Tanner stage I-5 }\end{array}$} & \multicolumn{2}{|c|}{ Prepuce and frenulum } \\
\hline Glans & \multicolumn{2}{|l|}{ Shaft } & \multicolumn{2}{|c|}{ Scrotum } \\
\hline Testes & \multicolumn{2}{|l|}{ Epididymus } & \multicolumn{2}{|c|}{ Vas deferens } \\
\hline Ulceration & \multicolumn{2}{|l|}{ Penile discharge } & \multicolumn{2}{|c|}{ Smegma } \\
\hline Presence of feces & \multicolumn{2}{|l|}{ Circumcision } & \multicolumn{2}{|c|}{ Urethral orifice } \\
\hline \multicolumn{5}{|l|}{ D. Conclusions } \\
\hline
\end{tabular}


Please use the sketches below to indicate injuries, inflammations or marks on the survivor's body.

\section{Sketch of person}

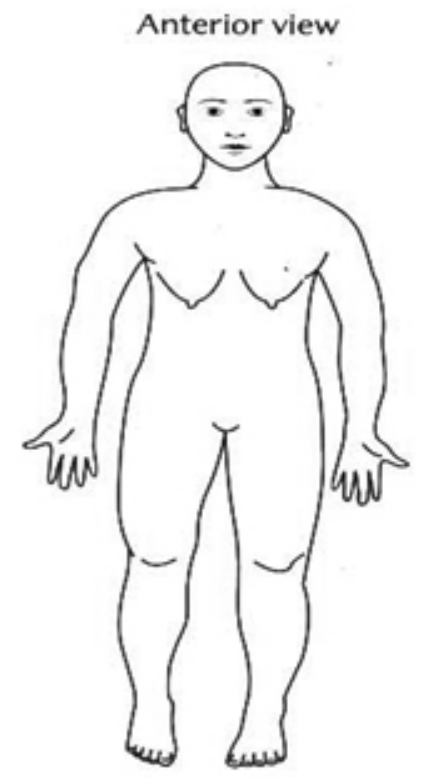

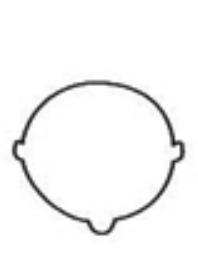

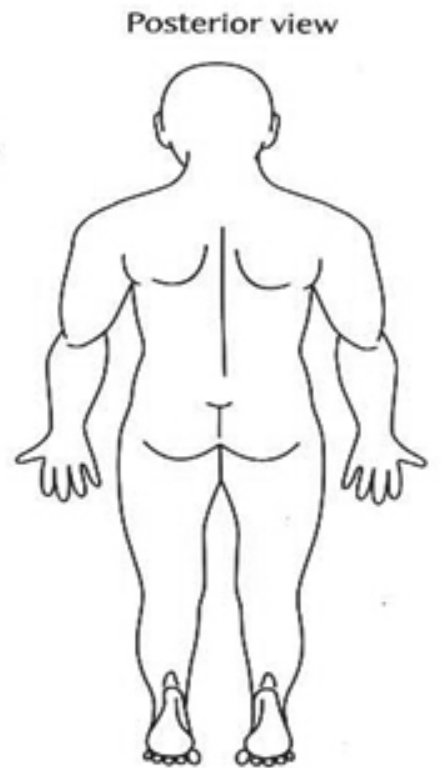

Comments

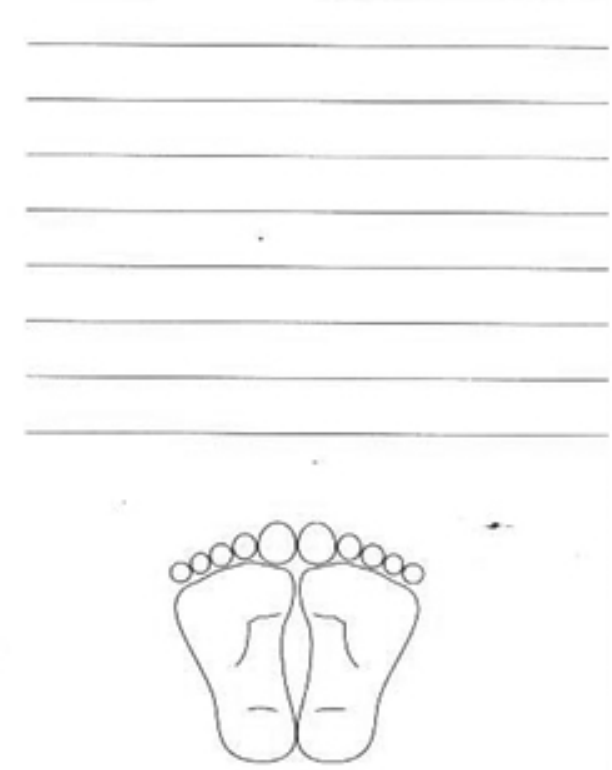

\section{Female Genitalia}
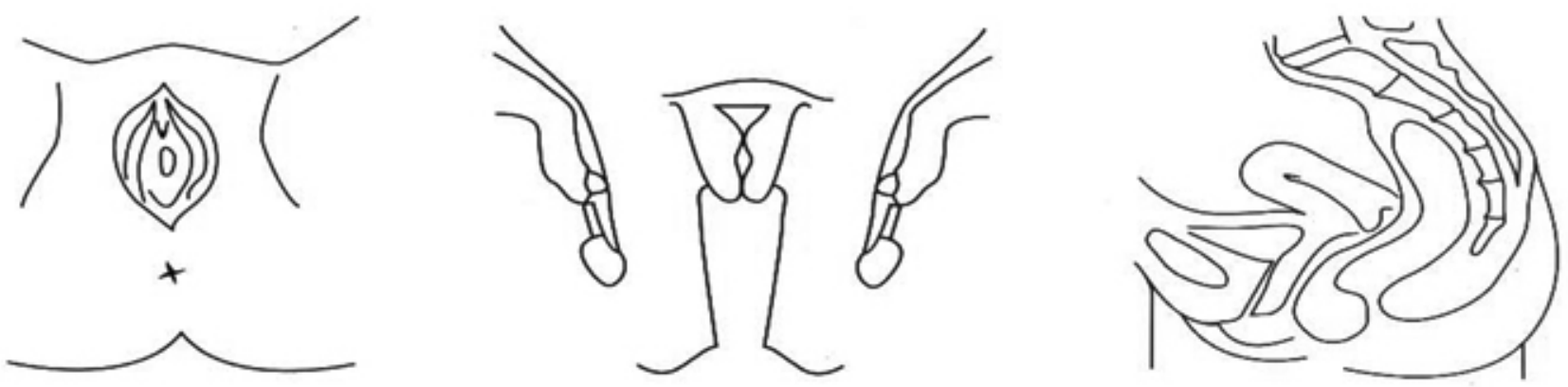

\section{Male Genitalia}
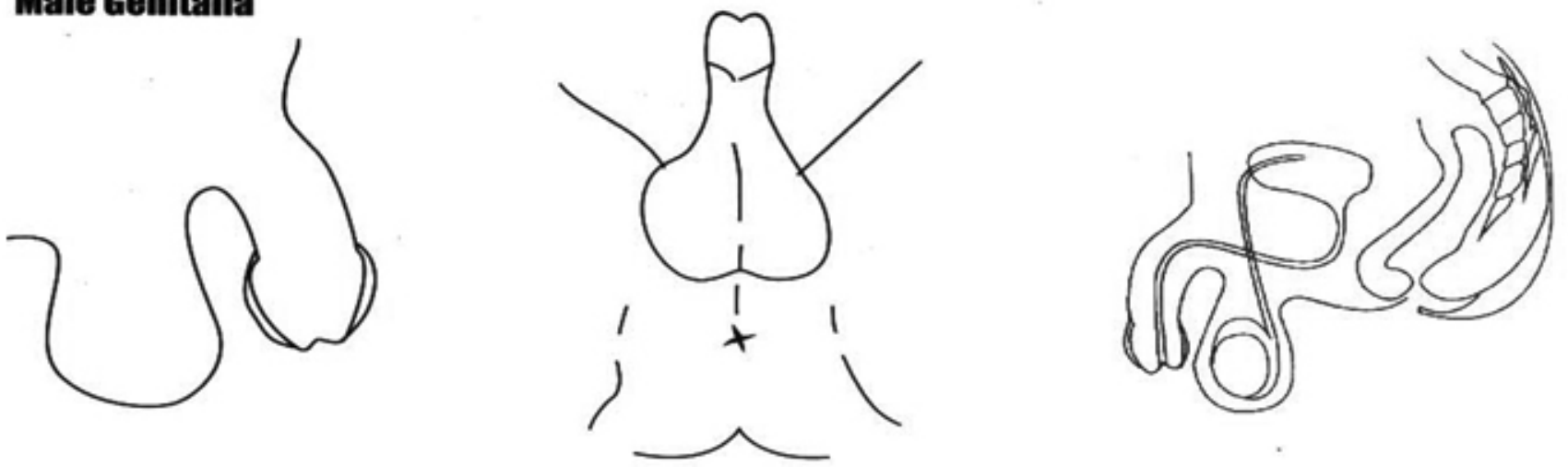
Section 6: Forensic Evidence Collection

\begin{tabular}{|l|l|l|l|}
\hline Type of evidence & Storage & $\begin{array}{l}\text { Person who collected the evidence } \\
\text { (Name, title, signature, date collected) }\end{array}$ & $\begin{array}{l}\text { Person to whom the provider } \\
\text { transferred evidence } \\
\text { (Name, title, date transferred) }\end{array}$ \\
\hline 32. Clothing & $\begin{array}{c}\text { Paper bag? } \\
\text { Y/N }\end{array}$ & & \\
\hline 33. Debris & $\begin{array}{c}\text { Paper bag? } \\
\text { Y/N }\end{array}$ & & \\
\hline 34. High Vaginal Swab & & & \\
\hline 35. Other & & & \\
\hline
\end{tabular}

\section{Section 7: Investigations}

\begin{tabular}{|l|l|l|l|l|l|}
\hline Investigations & A. Eligible? & B. Offered? & C. Received? & D. Department & Immediate Result/Comments \\
\hline 36. Pregnancy test & Y/N & Y/N & Y/N & & \\
\hline 37. Other & Y/N & Y/N & Y/N & & \\
\hline 38. Other & Y/N & Y/N & Y/N & & \\
\hline
\end{tabular}

D7. Total number of departments

\section{Section 8: HIV Services}

\begin{tabular}{|l|l|l|l|l|l|}
\hline & A. Eligible? & B. Offered? & C. Received? & D. Department & Comments \\
\hline $\begin{array}{l}\text { 39. Pre-test } \\
\text { counseling }\end{array}$ & Y/N & Y/N & Y/N & & \\
\hline 40. HIV test & Y/N & Y/N & Y/N & & $\begin{array}{l}\text { E. +/- (circle one) } \\
\text { F. Client given results? Y/N }\end{array}$ \\
\hline 4I. HIV PEP & Y/N & Y/N & Y/N & E. \# pills given: \\
\hline $\begin{array}{l}\text { 42. Referral to } \\
\text { PEP follow-up \& } \\
\text { adherence counseling }\end{array}$ & Y/N & Y/N & Y/N & & Follow-up date \\
\hline $\begin{array}{l}\text { 43. Referral of HIV+ } \\
\text { client to HIV/AIDS } \\
\text { care and treatment } \\
\text { services }\end{array}$ & Y/N & Y/N & Y/N & & \\
\hline
\end{tabular}

D8. Total number of departments 


\section{Section 9:Treatments}

\begin{tabular}{|l|l|l|l|l|l|}
\hline & A. Eligible? & B. Offered? & C. Received? & D. Department & Comments \\
\hline $\begin{array}{l}\text { 44. Medical treatment for } \\
\text { injuries }\end{array}$ & $\mathrm{Y} / \mathrm{N}$ & $\mathrm{Y} / \mathrm{N}$ & $\mathrm{Y} / \mathrm{N}$ & & \\
\hline 45. STI prophylaxis & $\mathrm{Y} / \mathrm{N}$ & $\mathrm{Y} / \mathrm{N}$ & $\mathrm{Y} / \mathrm{N}$ & & \\
\hline 46. HBV vaccine & $\mathrm{Y} / \mathrm{N}$ & $\mathrm{Y} / \mathrm{N}$ & $\mathrm{Y} / \mathrm{N}$ & & \\
\hline 47. Tetanus prophylaxis & $\mathrm{Y} / \mathrm{N}$ & $\mathrm{Y} / \mathrm{N}$ & $\mathrm{Y} / \mathrm{N}$ & & \\
\hline 48. EC & $\mathrm{Y} / \mathrm{N}$ & $\mathrm{Y} / \mathrm{N}$ & $\mathrm{Y} / \mathrm{N}$ & & \\
\hline 49. Trauma counseling & $\mathrm{Y} / \mathrm{N}$ & $\mathrm{Y} / \mathrm{N}$ & $\mathrm{Y} / \mathrm{N}$ & & \\
\hline 50. Other & $\mathrm{Y} / \mathrm{N}$ & $\mathrm{Y} / \mathrm{N}$ & $\mathrm{Y} / \mathrm{N}$ & & \\
\hline
\end{tabular}

D9. Total number of departments

\section{Section 10: Referrals}

\begin{tabular}{|l|l|l|l|l|}
\hline Type of service & $\begin{array}{l}\text { Referral } \\
\text { given? }\end{array}$ & $\begin{array}{l}\text { Referral services } \\
\text { organization \& contact } \\
\text { information }\end{array}$ & $\begin{array}{l}\text { Provider giving } \\
\text { referral (name, title, \& } \\
\text { department) }\end{array}$ & Comments: \\
\hline 5I. Police & Y/N & & & \\
\hline 52. Legal services & Y/N & & & \\
\hline 53. Counseling & Y/N & & & Specify type of service \\
\hline 54. Other & Y/N & & & \\
\hline
\end{tabular}

\section{Section I I: Follow-up Care at This Health Facility}

\begin{tabular}{|l|l|l|l|}
\hline & $\begin{array}{l}\text { Recommended } \\
\text { visit date }\end{array}$ & Date of visit & Services provided and comments: \\
\hline 55. I week follow-up & & & \\
\hline 56. Other follow-up & & & \\
\hline 57. 3-month HIV test & & & \\
\hline 58. 6-month HIV test & & & \\
\hline
\end{tabular}

\footnotetext{
*Adapted from: the Kenyan Ministry of Health PRCI Form, the South African J88 form, and the "SV Examination Record"template (contained in World Health Organization. 2003. Guidelines for medico-legal care for victims of sexual violence. Geneva:World Health Organization. Geneva:World Health Organization.).
} 


\section{APPENDIX 6}

\section{SAMPLE COMMUNITY FGDTOOL}

Date: Start Time: End Time:

Community/Pilot Site:

Type of Group Interviewed (local leaders, men, women, etc.)

\# of Women: \# of Men: \# of Children: Total \#:

Name of Facilitator/s:

\section{Introduction}

\section{INTRODUCE MODERATORS, TRANSLATORS, RECORD KEEPERS}

\section{INTRODUCE TOPIC OF RESEARCH}

I am interested in learning about some of the concerns and needs of people related to violence against women in this community. I'm especially interested in trying to understand your community's attitudes related to sexual violence that women and girls and how those attitudes. I am also interested in the availability and quality of services for victims of sexual violence. I hope that your answers to my questions will help improve services for women, girls, and families in this community. I expect our discussion to last about one to one-anda-half hours.

\section{SECURE INFORMED CONSENT}

Assure participants that any information they disclose will be confidential. That is, any outputs and learning from the discussion sessions will be shared in ways that do not identify any particular individual. Note that in later parts of the process individuals may want to share their own experiences, but that would be their own decision. If facilitators share information from the sessions, they will do so in a general way, where individual stories or identities could not be connected to any individual person in the community.

If the session is being recorded, note that the tapes will be used only to aid the recorder in capturing the discussion.

Finally, tell participants that if they don't wish/no longer wish to participate in the study for any reason, they may withdraw at any time. Encourage them to ask any questions they have.

Do you agree to be interviewed? 
NOTE WHETHER RESPONDENTS AGREE TO INTERVIEW.

[ ] AGREES TO BE INTERVIEWED.

[ ] DOES NOT AGREE TO BE INTERVIEWED

Please record the number of people who do not agree.

TO BE COMPLETED BY INTERVIEWER

I CERTIFY THAT I HAVE READ THE ABOVE CONSENT PROCEDURE TO THE GROUP.

SIGNED:

4. ESTABLISH GROUND RULES

- Everybody's ideas have merit

- No judgment or discussion of other's ideas

- One person to speak at a time

- Ideas (contributions) are anonymous. Please do not name any names.

\section{FIRST, I WOULD LIKE TO ASK YOU ASK YOU SOME QUESTIONS ABOUT THE SAFETY AND SECURITY OF WOMEN AND GIRLS:}

I. Are you aware of problems with safety of individuals, particularly, women and girls in this community? (Ask for examples. If no one speaks specifically about GBV, evaluate the group to decide whether you want to bring up the issue now or wait until the group has developed more comfort talking about these issues.)

2. What are the circumstances that cause problems of safety and security for individuals in this community? (Ask for examples.)

3. What are the specific forms of violence against women and girls? What practices are considered sexually inappropriate, abusive, or violent in the community? Can you give examples of sexual abuse in your community? (Examine definitions of forced sex/rape, sexual harassment, sexual manipulation, etc.)

4. When a husband insists on sex from his wife, does she have the right to refuse sex? If she does refuse and he forces her to have sex, is that rape? 


\section{ESTABLISH CONSENSUS ON DEFINITION OF SEXUAL VIOLENCE BY SUMMARIZING WHAT PEOPLE HAVE IDENTIFIED AS DIFFERENT TYPES OF SEXUAL VIOLENCE. NAME TYPES AND ASK IF EVERYONE AGREES.}

5. When and where does sexual violence occur? Without mentioning names or indicating anyone specific, who are the perpetrators?

6. Without mentioning names or indicating anyone specific, which groups (of women) do you think feel the least safe, or feel at most risk for sexual violence?

PROBE: If participants do not mention them, ask:What about young girls and boys? Are they at risk for violence by strangers and acquaintances? What about sex workers? Can they experience violence by potential or existing clients? What about women in relationships? Can they experience sexual violence/rape by their partners?

\section{NOW I WOULD LIKE TO ASK YOU ABOUT SERVICES AND SOURCES OF SUPPORT FOR WOMEN AND GIRLS IN YOUR COMMUNITIES.}

I. Who are survivors most likely to tell about their experience of sexual violence? PROBE: Among these people, who are they most likely to tell? Why? Why not? For any of the following that haven't been named, from whom would women seek help? (family members, other women, health worker, community leader, police/security people/authorities, someone else) Why? Why not?

PROBE:Are there any differences in whom survivors look to for help when: rural vs. urban; child vs. adult; boy vs. girl; woman raped by husband vs. raped by stranger.

Note to facilitator: include here specific characteristics of women that may result in different help-seeking experiences.

2. For women who have experienced sexual violence/assault, what help do women look for, if any? If so, what type of assistance do they seek? Without naming names, do you know any women who has experienced violence and sought help? What type of help did she seek? Do you know if she got tested for HIV?

3. What services were available for this kind of thing? What people or groups in this community are involved in helping those most in need? What social and legal services exist to help address problems associated with violence (e.g., health, police, legal counseling, social counseling)? Who provides these services? Who do you think will be the right person(s) to help women who are in abusive relationships? Are these services linked together, i.e., are there support networks for survivors of sexual violence?

4. When a woman goes to those services, how do they respond to her? How does the community respond when they hear about a case of sexual violence? What challenges does she have while receiving services? PROBE: Are there any differences in barrier for help when: rural vs. urban; child vs. adult; boy vs. girl; woman raped by husband vs. raped by stranger. 
5. What are challenges to accessing these services to deal with a problem related to sexual violence? How might these barriers be overcome? What are the current supports? How could these efforts be improved?

PROBE:Are there any differences in barrier for help when: rural vs. urban; child vs. adult; boy vs. girl; woman raped by husband vs. raped by stranger?

\section{CLOSING QUESTIONS:}

Before we finish, I would like to hear what you think should be done to support victims of sexual violence in $[\ldots]$ ?

Do you have any other thoughts or comments that you would like to share?

\section{CLOSE THE INTERVIEW:}

Thank you all for your time and ideas. This has been extremely helpful. As I said in the beginning, the purpose of this discussion was to help me learn about the needs of women in your community in relation to gender-based violence. As more services are developed here, we want to be sure they help you address the problems you are facing.

Please remember that you agreed to keep this discussion confidential. Please do not share with others the details of what was said here. People will be curious and you may have to say something-I suggest you tell them that I was asking questions about women and men and health issues, just gathering information-like l'm sure has happened before. Please do not give details of what was said here, so that we can try to preserve confidentiality and the safety of people who are exposed to violence.

How does that sound to you? Do you have questions for me? If anyone would like to speak with me in private, I will stay here after we end.

Thank you for your help.

Adapted from RHRC Consortium. 2003. “Gender-based violence tools manual: For assessment \& program design, monitoring \& evaluation in conflict-affected settings.” New York: RHRC Consortium.Available at:http://www.rhrc.org/resources/gbv/gbv_toolsh manual_toc.htm 


\section{APPENDIX 7}

\section{MINIMUM SUPPLIES NEEDED TO PROVIDE SV SERVICES}

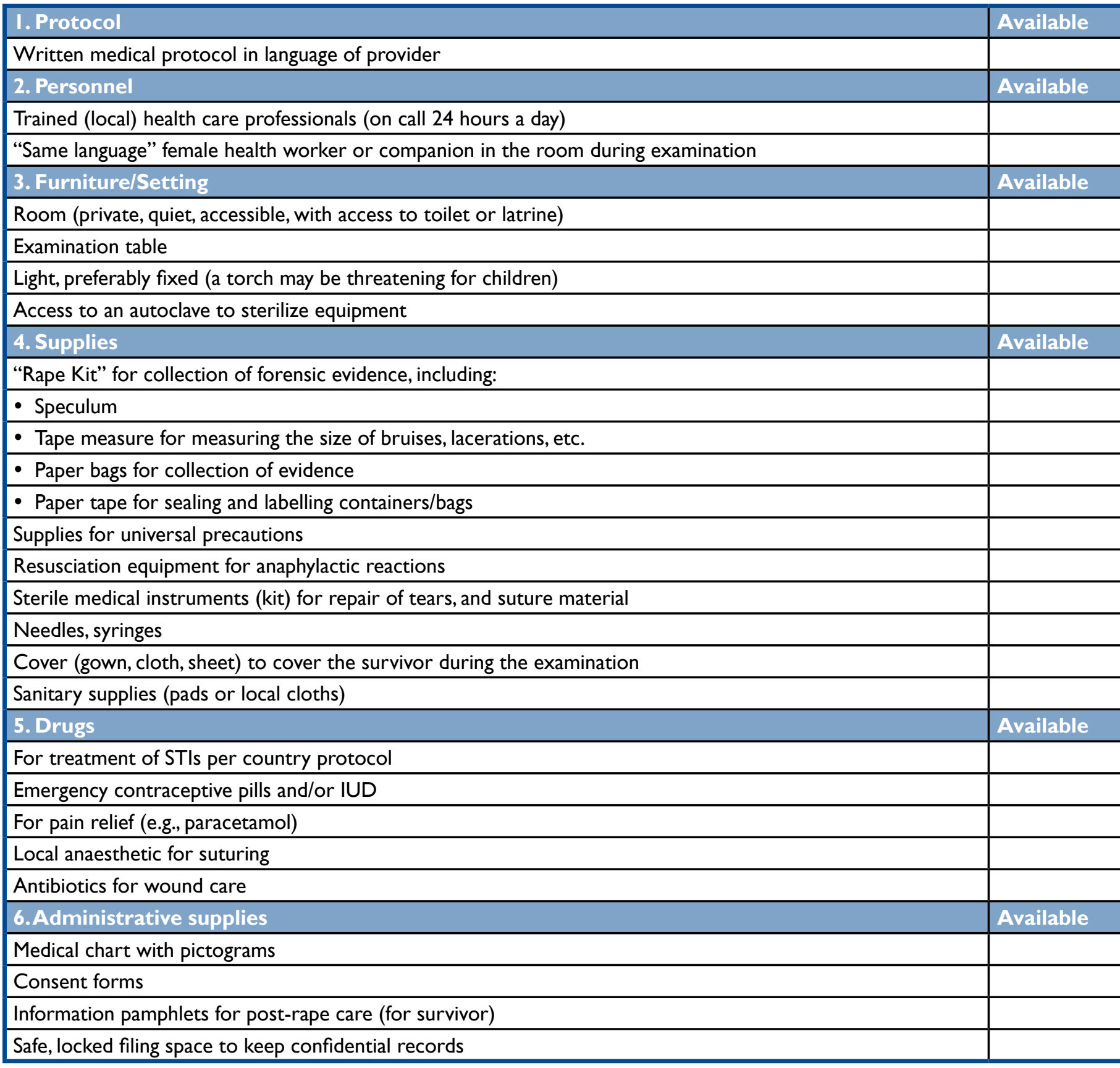

Source:WHO, United Nations Population Fund and United Nations High Commissioner for Refugees. 2004 (revised edition). "Clinical management of rape survivors: Developing protocols for use with refugees and internally displaced persons." Geneva: WHO. 


\section{APPENDIX 8}

\section{PATIENT INFORMATION BROCHURE, KENYA}

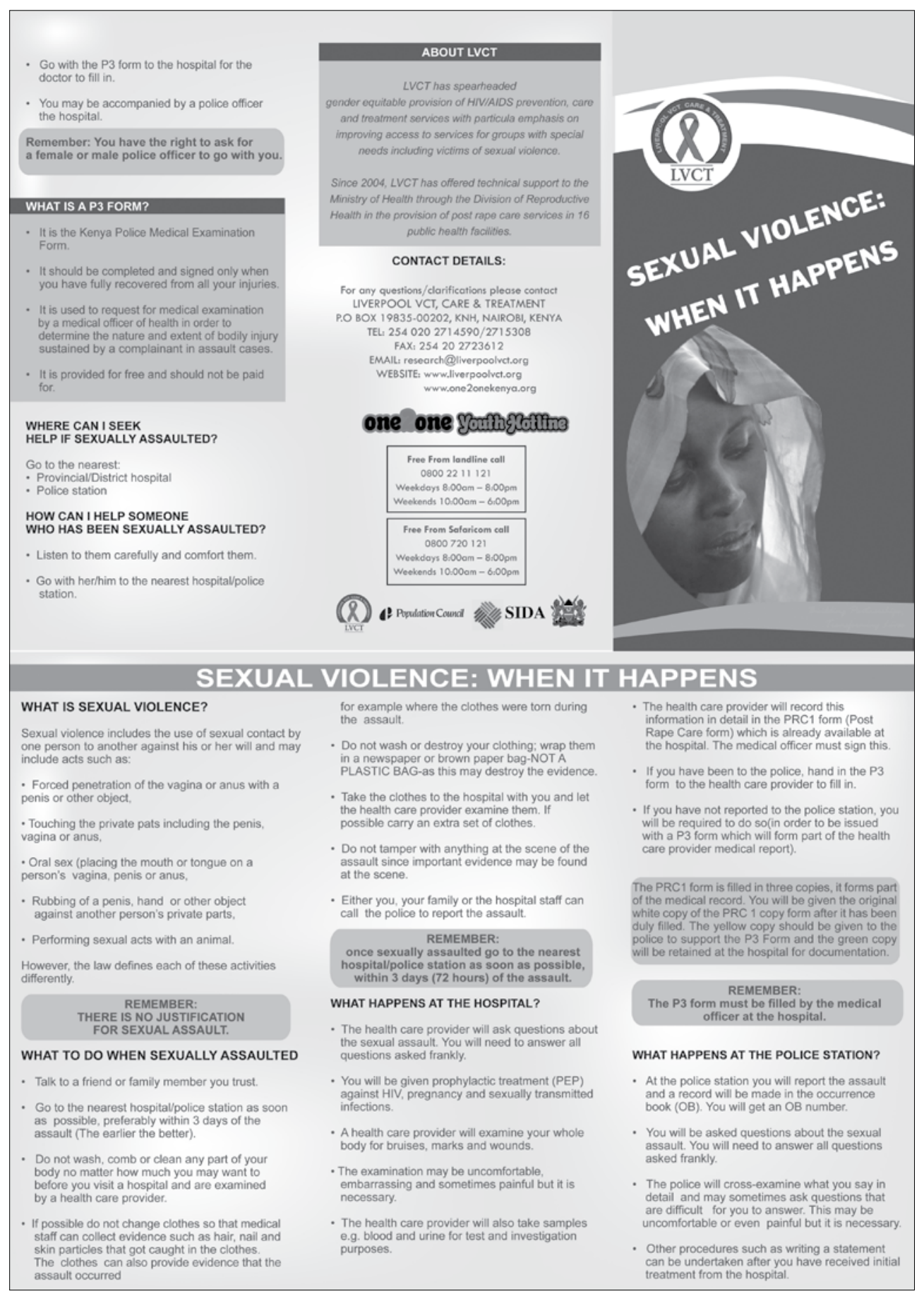




\section{APPENDIX 9}

\section{SAMPLE CLIENT FLOW CHART, MULAGO HOSPITAL, KAMPALA, UGANDA}

\section{CLIENT FLOW FOR SGBV SURVIVORS IN MULAGO HOSPITAL}

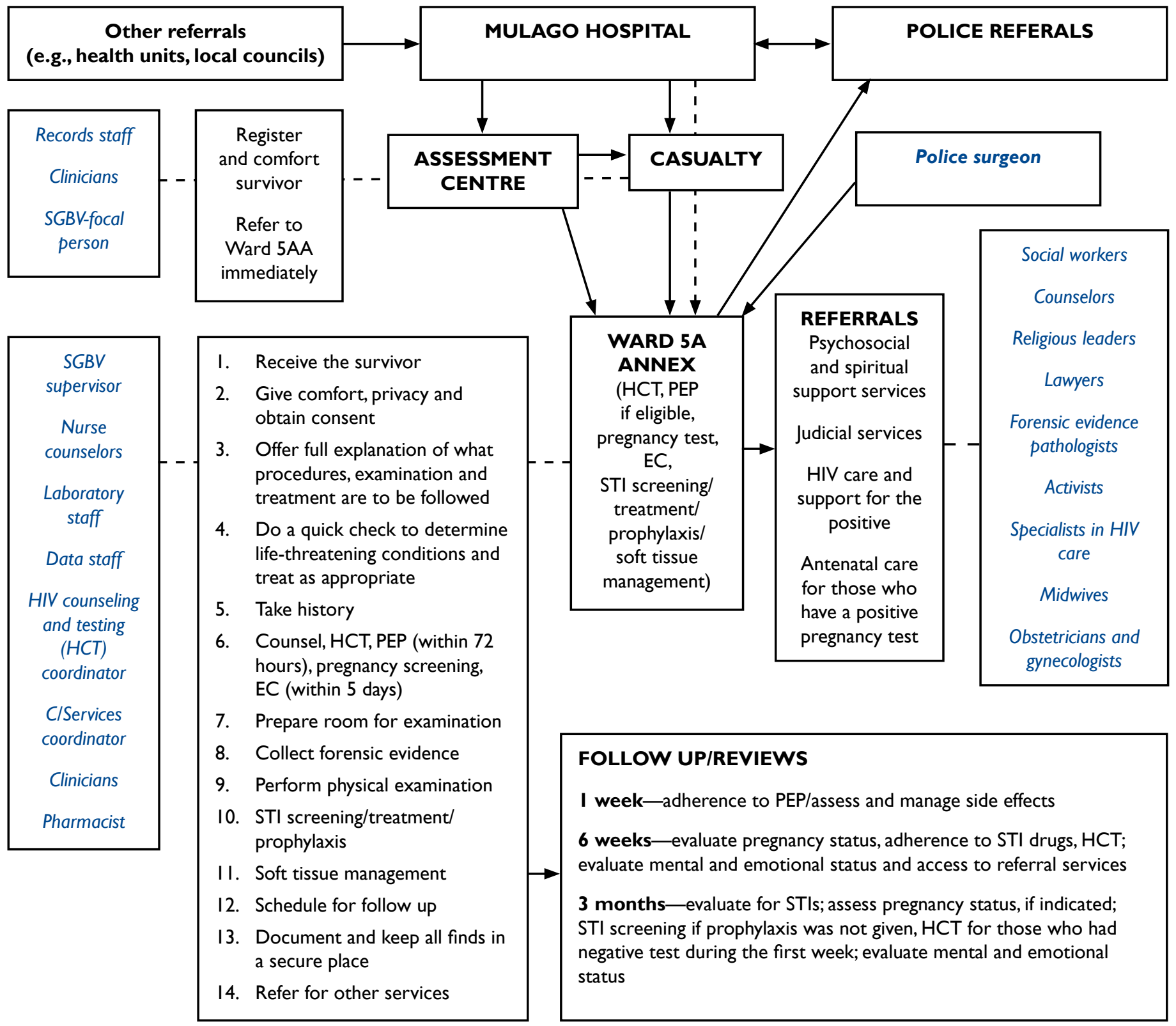




\section{APPENDIX 10}

\section{AWARENESS-RAISING POSTER DEVELOPED FOR USE WITHIN UGANDAN COMMUNITIES}

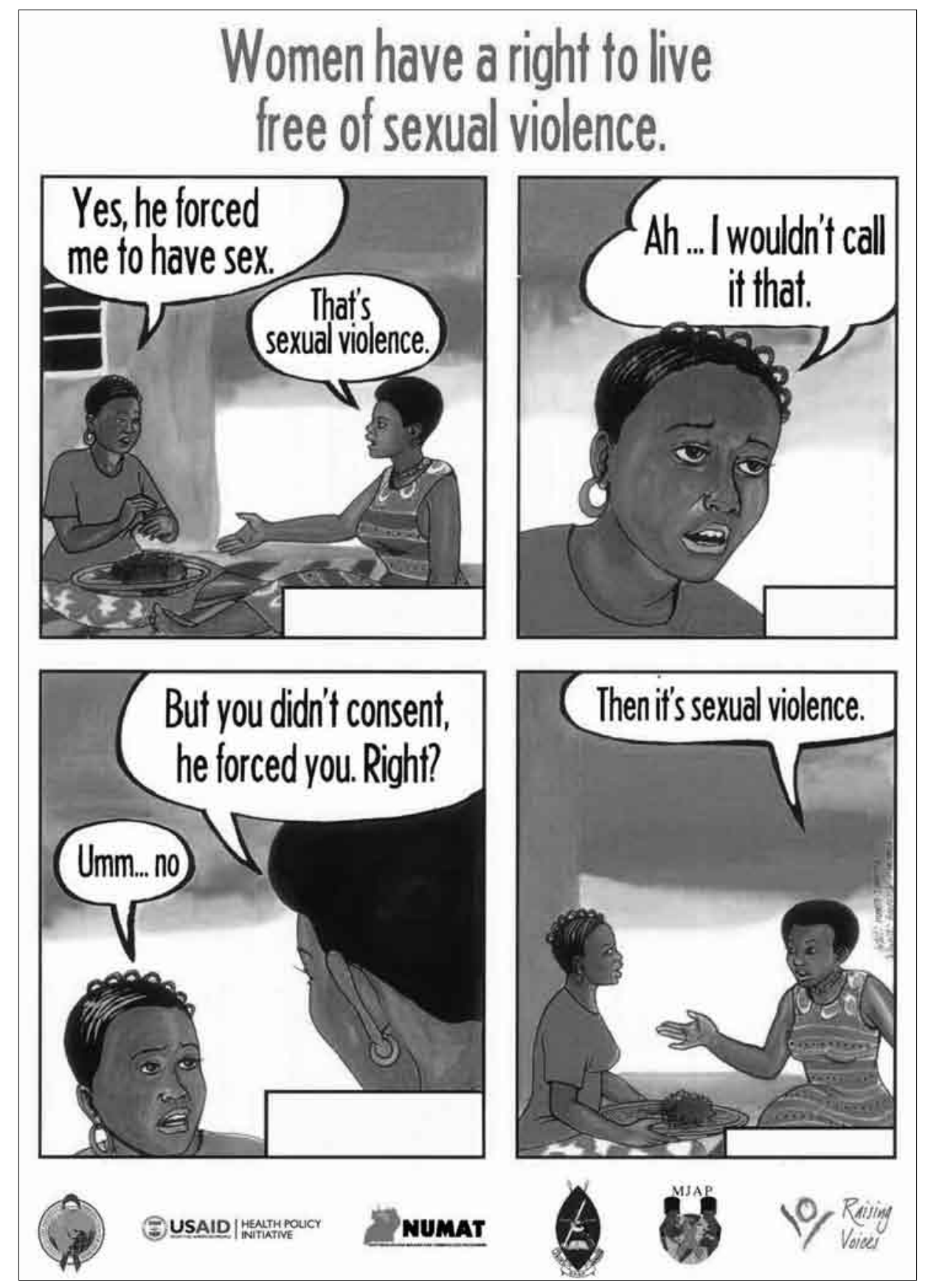




\section{APPENDIX I I}

\section{AWARENESS-RAISING POSTERS DEVELOPED FOR USE WITHIN UGANDAN HEALTH FACILITIES}
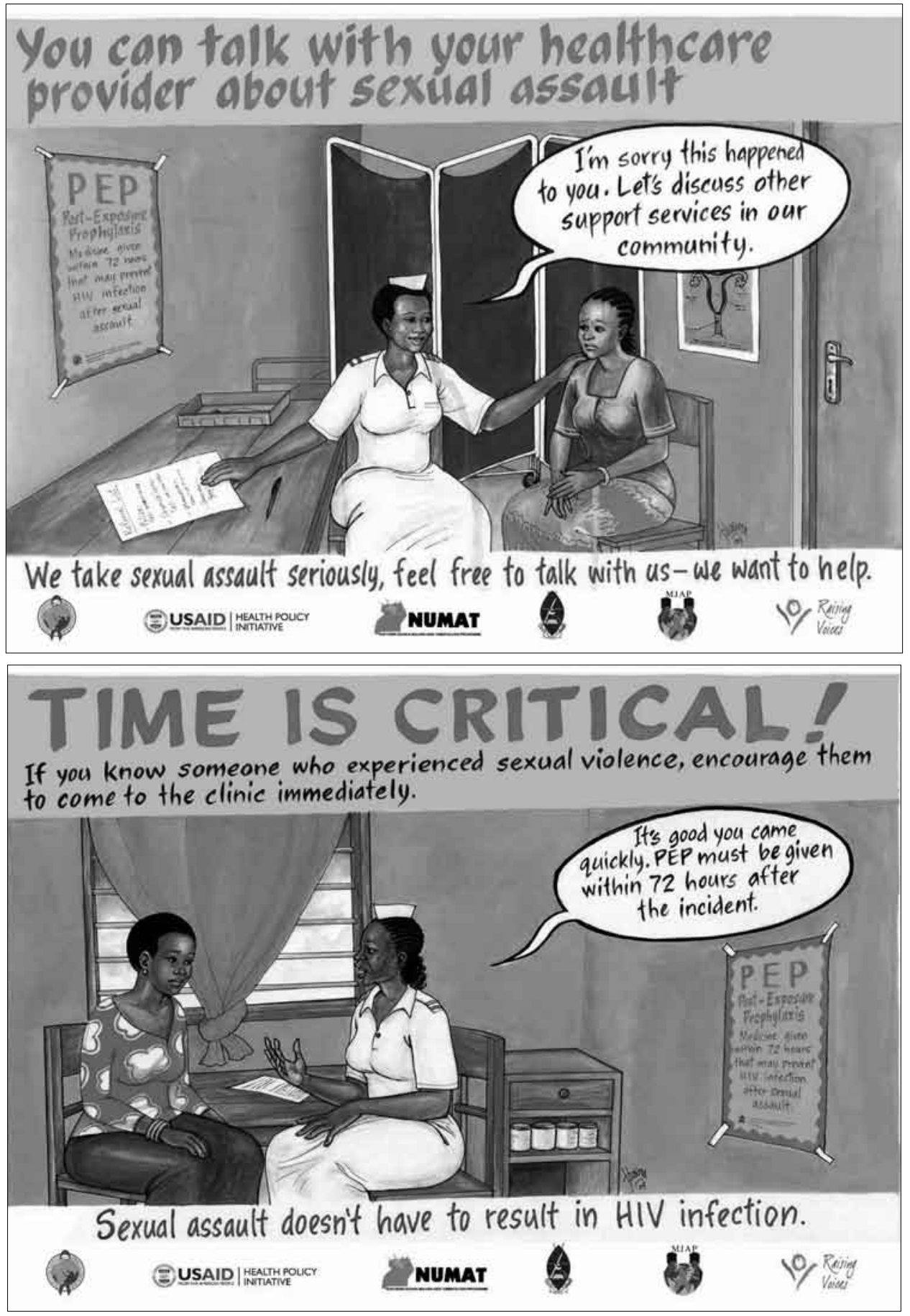

U.S. Agency for International Development I 300 Pennsylvania Avenue, NW

Washington, DC 20523

Tel: (202) 7I2-0000

Fax: (202) 216-3524

www.usaid.gov 